\title{
Quantitative Multiplexed Multi-pinhole Small-Animal SPECT
}

by

Jared H Strydhorst

A thesis submitted to the Faculty of Graduate and Postdoctoral Affairs in partial fulfillment of the requirements for the degree of

Doctor of Philosophy

in

Physics

Carleton University

Ottawa, Ontario

(C) 2013, Jared Strydhorst 


\section{Abstract}

Small-animal microSPECT is an important research tool for the development of clinical diagnostic tests, evaluation of novel pharmaceuticals, and basic science research. Experimentally quantifying the accuracy of microSPECT - the reproducibility of measurements made using microSPECT, and how well the reconstructed microSPECT data correlates to the true tracer distribution - is therefore essential. Photon attenuation and scatter complicate the problem of acquiring quantitatively accurate information from SPECT.

In this work, the reproducibility of cardiac measurements made in rats using the nanoSPECT/CT scanner (Bioscan, Washington, DC) is first evaluated without scatter or attenuation correction. For relative perfusion measurements of rat myocardia, normal databases for ${ }^{99 \mathrm{~m}} \mathrm{Tc}$-tetrofosmin and ${ }^{201} \mathrm{Tl}$ imaging are created and it is shown that the relative myocardial perfusion measurements made using either tracer are reproducible over repeated scans and over multiple rats with standard deviations of $3 \%$ and $4 \%$ respectively.

Attenuation and scatter compensation are implemented in the SPECT reconstruction algorithm to evaluate the accuracy of using nanoSPECT to recover absolute tracer concentrations in phantoms and in vivo. With attenuation and scatter compensation, absolute tracer concentration is measured using SPECT images with systematic and random uncertainties as low as $1 \%$ in phantoms, relative to dose calibrator measurements. The systematic and random uncertainties for measurements of total cardiac tracer uptake in vivo compared to well chamber measurements ex vivo were as small as $-1 \%$ and $3 \%$ respectively.

Reevaluation of the reproducibility of the cardiac measurements with attenuation and scatter correction demonstrates that neither correction strongly influences the relative perfusion measurements or their reproducibility. However, it is shown that attenuation correction does significantly modify the relationship between body weight and absolute tracer uptake. 


\section{Acknowledgements}

I would like thank to my supervisor, Dr. Glenn Wells for his guidance and critique of this work. I would also like to acknowledge the contributions of Dr. Terry Ruddy and Dr. Frans Leenen who collaborated with Dr. Wells and me on the paper which became Chapter 4 of this thesis.

Thanks also to Dr. Tong Xu for reading and providing feedback on a draft of this thesis.

My thanks also to Julia Lockwood and Myra Kordos for their assistance with handling the animals and acquiring many of the SPECT scans which contributed to this work. Finally, thanks also to Lihui Wei for her assistance with the well counter measurements for in vivo quantification. 


\section{Statement of Originality}

Parts of the work on reproducibility (Chapter 4) have been presented at the Society of Nuclear Medicine 2010 (Salt Lake City):

J Strydhorst, RG Wells, "Reproducibility of Tc99m-tetrofosmin cardiac gated microSPECT measurements in rats"

This work was also published in the Journal of Nuclear Medicine. My contribution was the data analysis and writing, Dr. Leenen supervised the rat surgeries, and Dr. Wells was responsible for the overall experimental design.

JH Strydhorst, TD Ruddy, FH Leenen, RG Wells, "Reproducibility of Serial Left Ventricle Perfusion, Volume, and Ejection Fraction Measurements Using Multiplexed Multipinhole SPECT in Healthy Rats and Rats after Myocardial Infarction" J Nucl Med 52 (2011):1285-1292.

Various stages of the work on attenuation corrected multiplexed multi-pinhole SPECT was presented at various conferences. This thesis includes material presented as poster at the Canadian Organization of Medical Physicists (COMP) Annual Scientific Meeting 2010 (June 16-19, 2010, Ottawa, ON):

J Strydhorst, RG Wells. "Attenuation Correction for 99mTc-Tetrofosmin Micro SPECT/CT Cardiac Measurements in Rats",

as a poster at IEEE Medical Imaging Conference 2010 (October 20 - Nov 6, 2010, Knoxville, TN): J Strydhorst, RG Wells, "Attenuation Correction of Multiplexed Multi-Pinhole microSPECT Reconstruction", in an oral presentation to the COMP/AAPM Joint Conference 2011 (July 31 - Aug 4, 2011, Vancouver, BC):

J Strydhorst, RG Wells, "Quantitative Reconstruction of Multiplexed Multi-Pinhole SPECT with Scatter and Attenuation Correction",

and as a poster at the IEEE Medical Imaging Conference 2011 (Oct 23-29, 2011, Valencia, Spain):

J Strydhorst, RG Wells, "Quantitative OS-EM reconstruction of multiplexed multi-pinhole cardiac SPECT".

For all of the work on reconstruction, attenuation and scatter correction, I wrote the reconstruction software, did most of the experimental work, and did the analysis.

We plan to submit the work on quantitative multiplexed multi-pinhole SPECT and the work reevaluating the cardiac measurements with attenuation and scatter correction as papers. 


\section{Table of Contents}

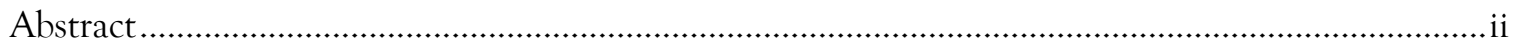

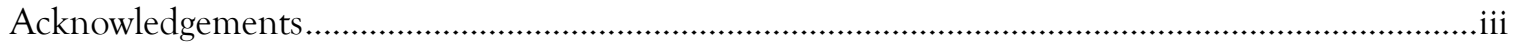

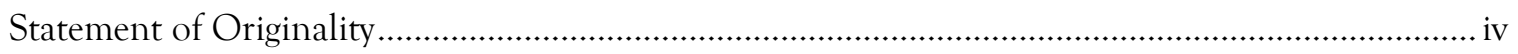

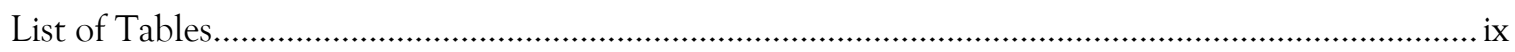

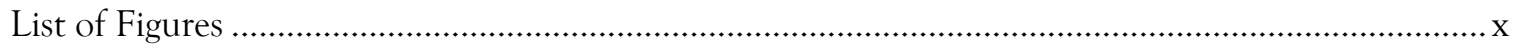

Abbreviations and Notation .......................................................................................................

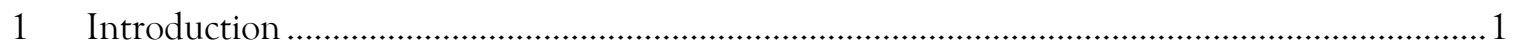

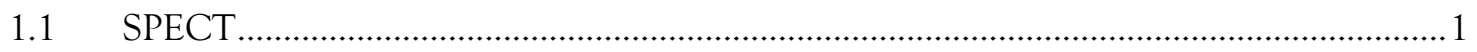

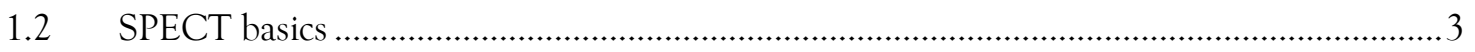

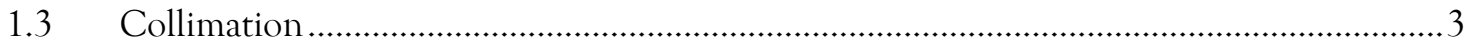

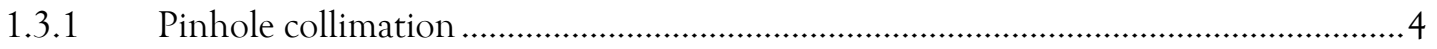

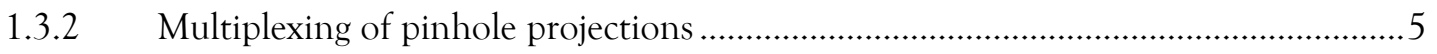

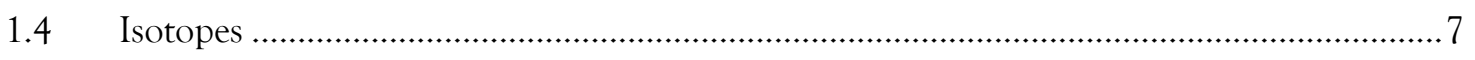

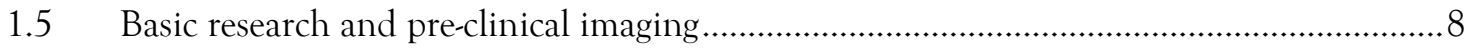

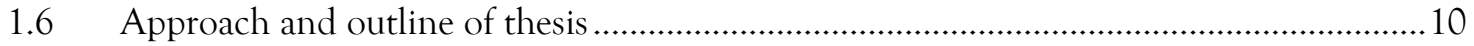

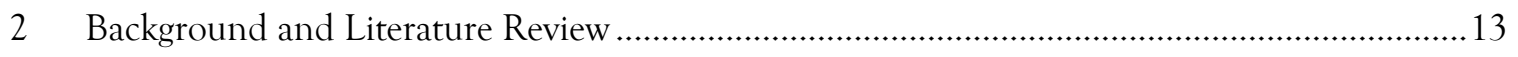

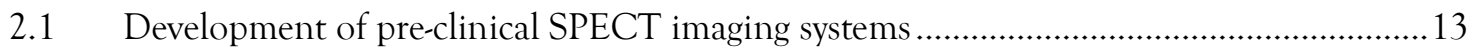

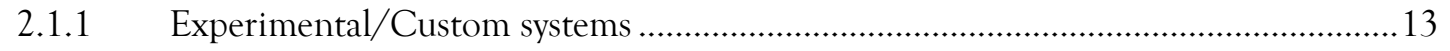

2.1.2 Commercial small animal systems ............................................................................18

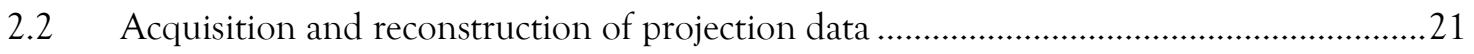

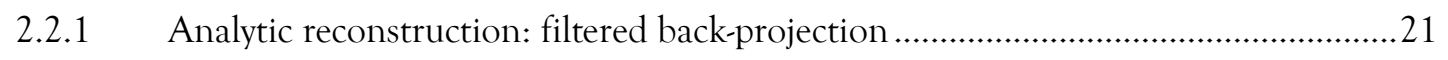

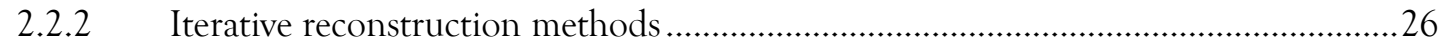

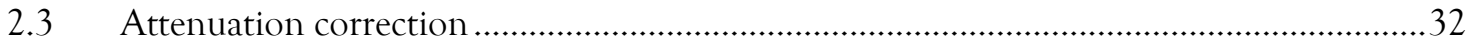

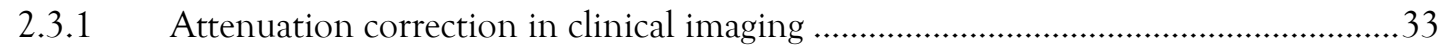

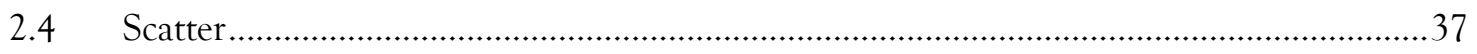

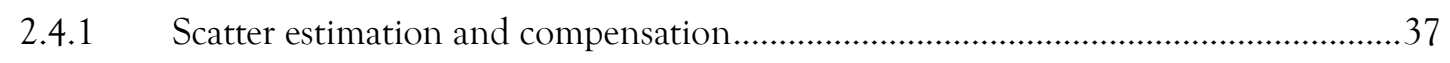

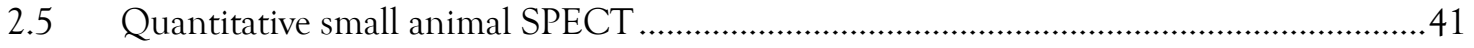

3 Acquisition and Reconstruction of SPECT/CT Images ............................................................ 46

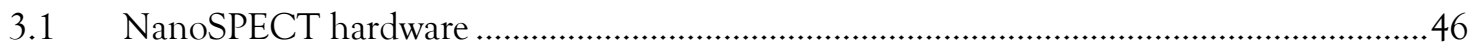




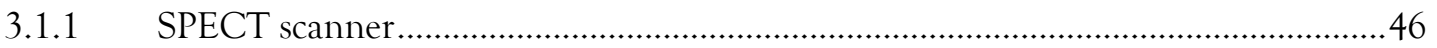

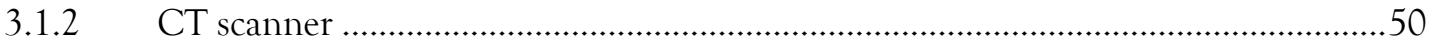

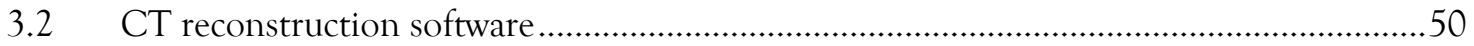

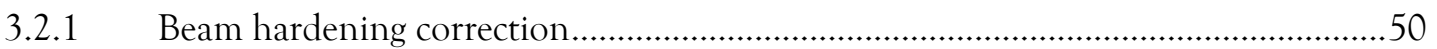

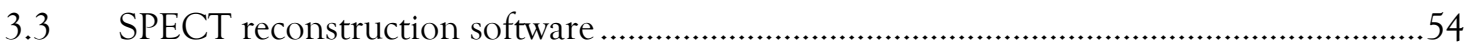

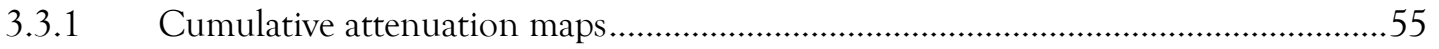

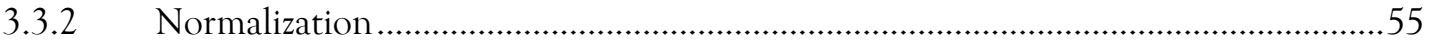

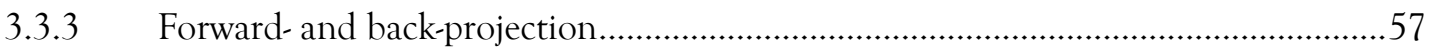

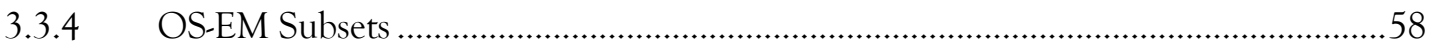

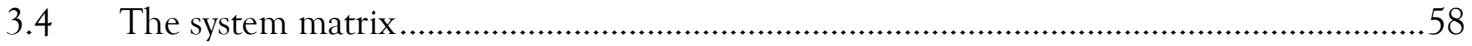

3.5 Maximum object size with multiplexed projections ……....................................................61

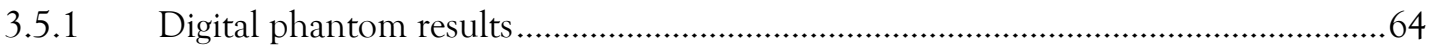

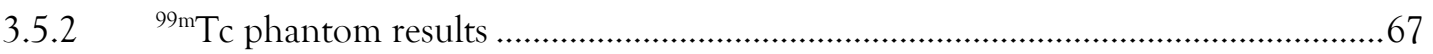

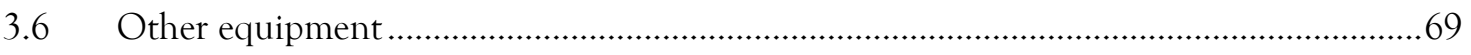

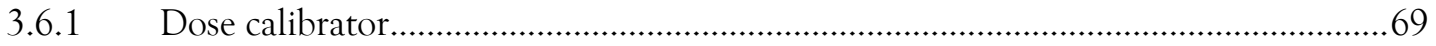

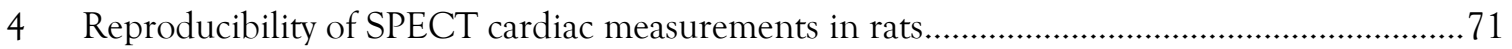

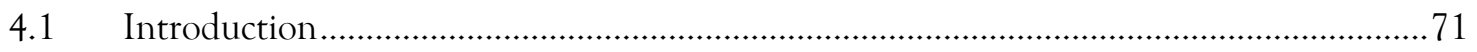

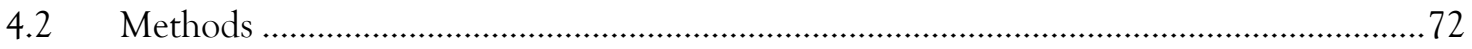

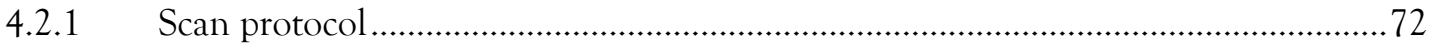

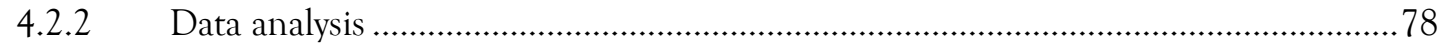

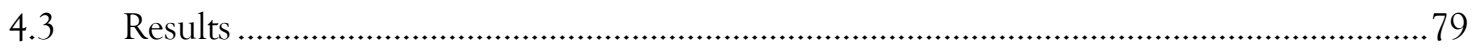

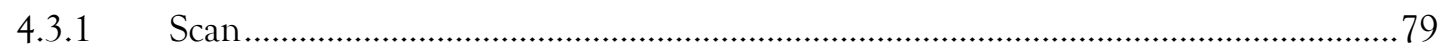

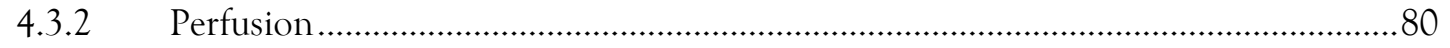

4.3.3 Reproducibility of infarct severity measurements .........................................................83

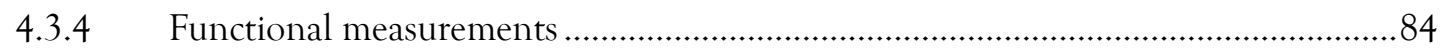

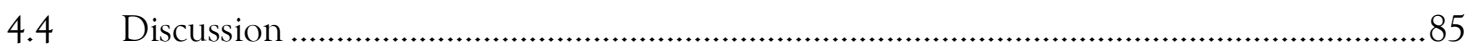

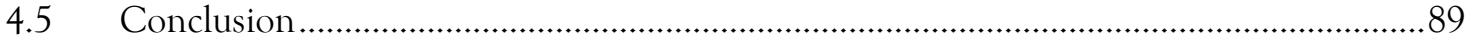

5 Attenuation and Scatter Correction of pre-clinical SPECT Data ...............................................91

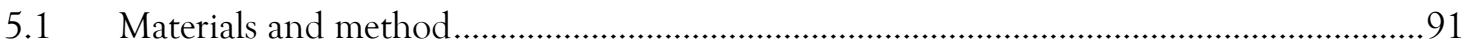

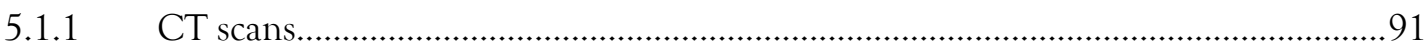

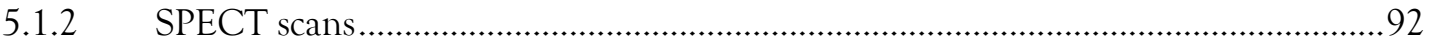




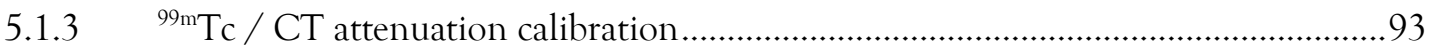

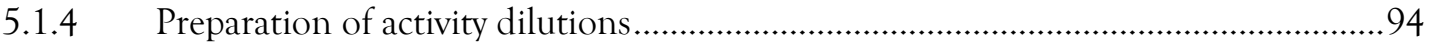

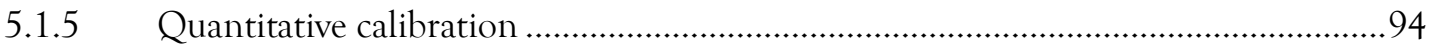

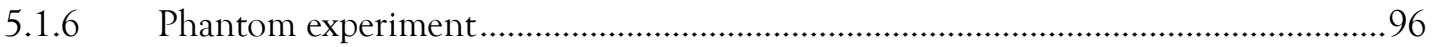

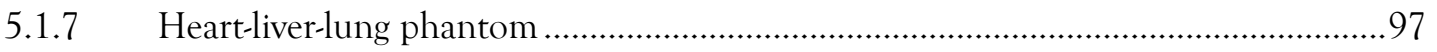

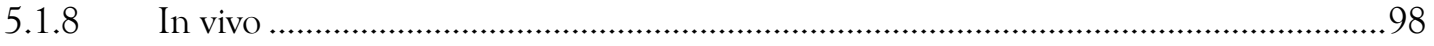

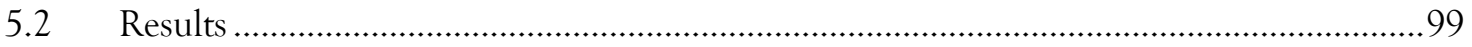

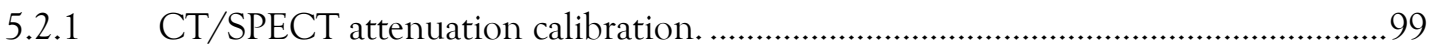

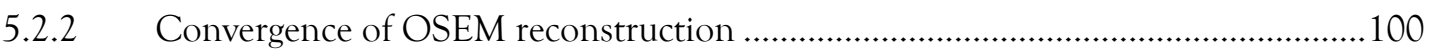

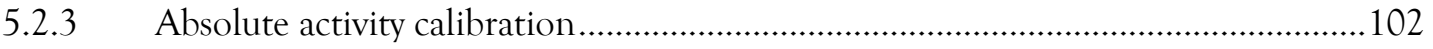

5.2.4 Amount of scatter and choice of DEW scatter coefficient, k ..................................102

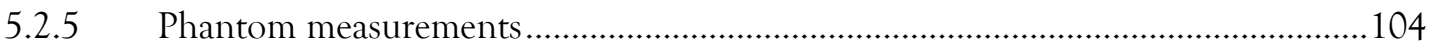

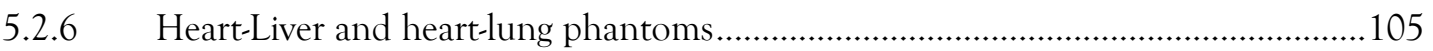

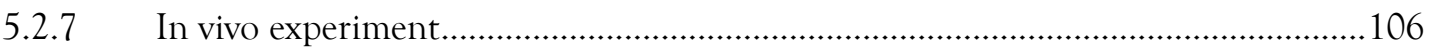

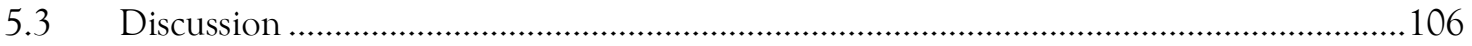

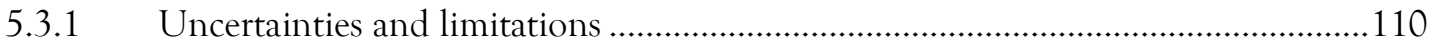

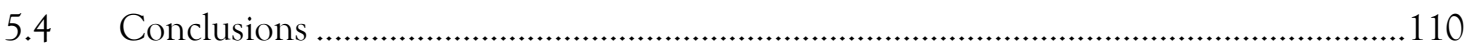

6 Reproducibility of cardiac rat SPECT data with attenuation correction ...................................112

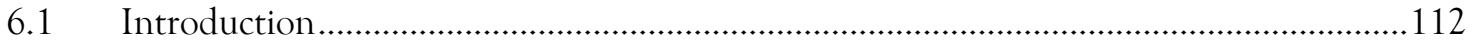

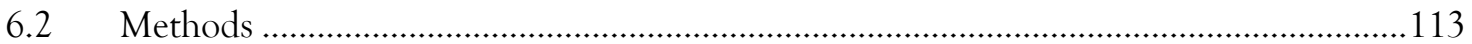

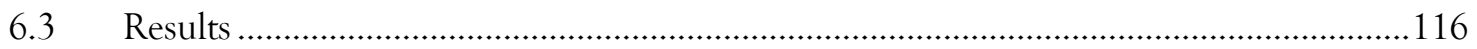

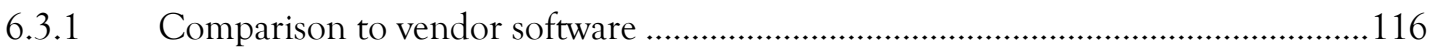

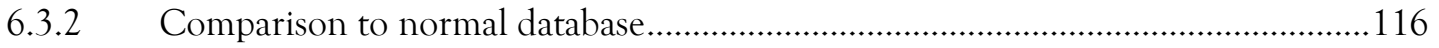

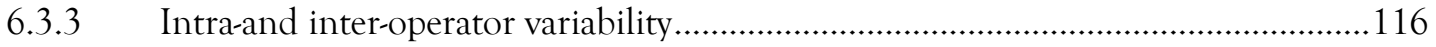

6.3.4 Effect of attenuation correction on relative perfusion ...............................................116

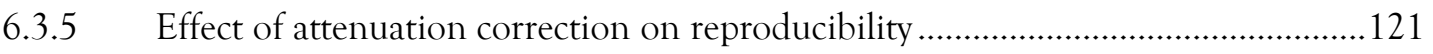

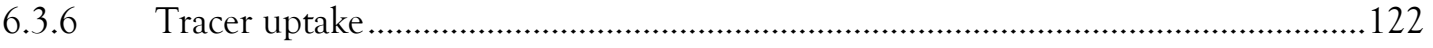

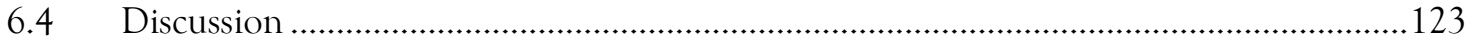

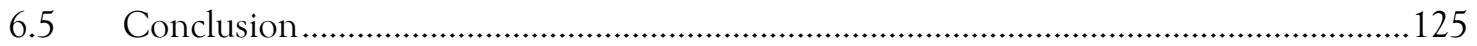

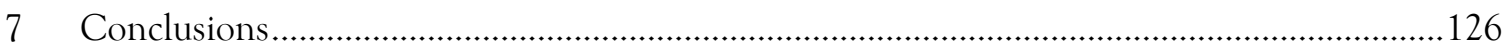

7.1 Reproducibility of rat cardiac measurements using microSPECT ..................................126

vii 


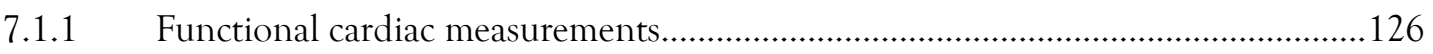

7.1.2 Semi-quantitative cardiac perfusion measurements ....................................................126

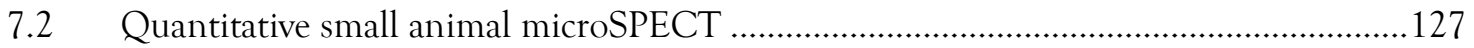

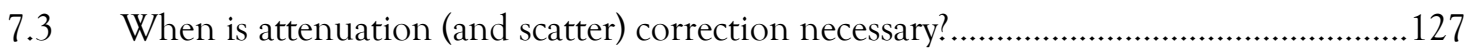

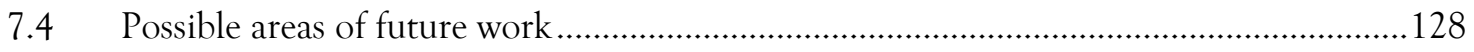

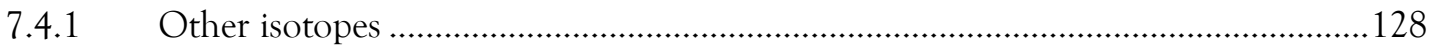

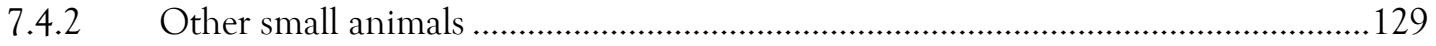

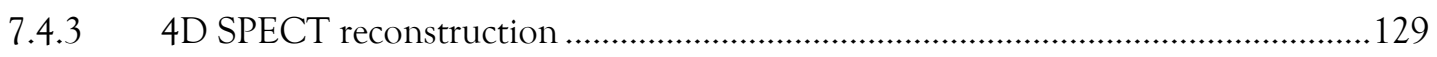

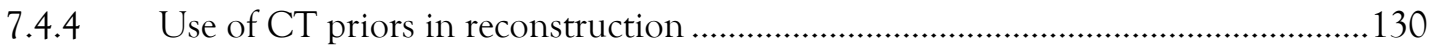

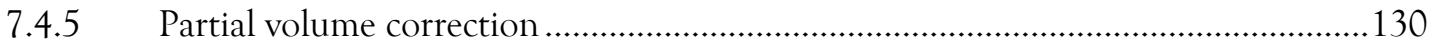

7.4.6 Analytical assessment of multiplexed multi-pinhole field of view ............................130

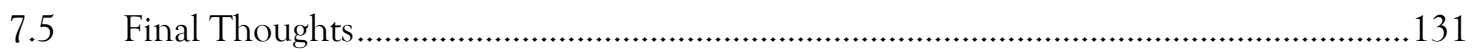

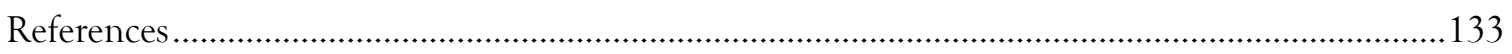




\section{List of Tables}

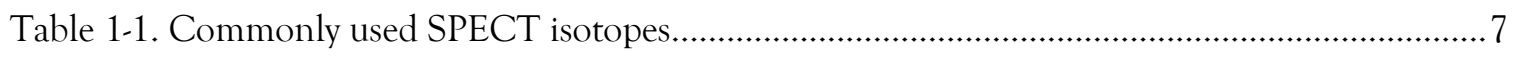

Table 4-1. Reproducibility of cardiac perfusion measurements in normal rats ................................82

Table 4-2. Reproducibility of cardiac perfusion measurements in myocardial infarct rats ...............82

Table 4-3. Average combined $z$-scores for five scans of eight infarcted rats. ....................................83

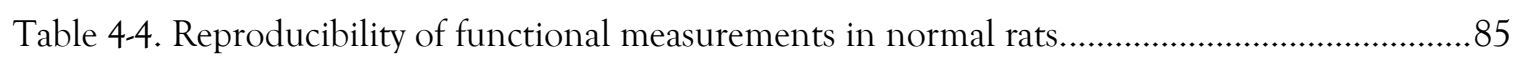

Table 4-5. Reproducibility of functional measurements in myocardial infarct rats...........................85

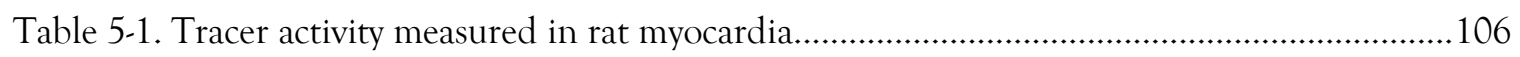

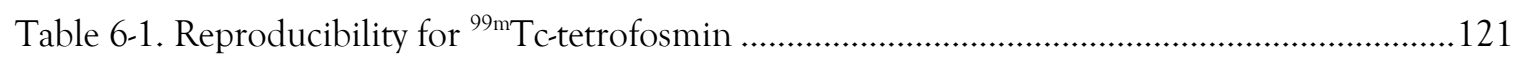

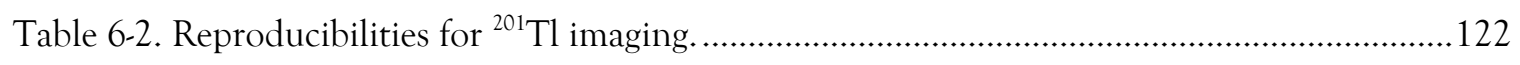




\section{List of Figures}

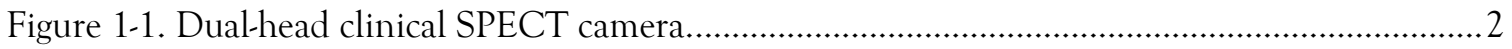

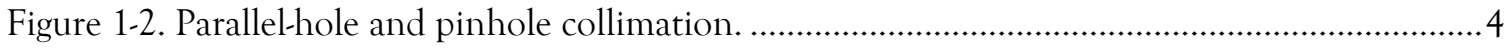

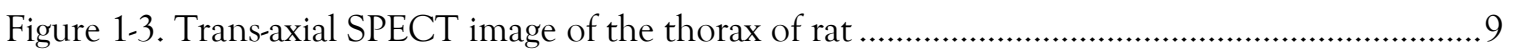

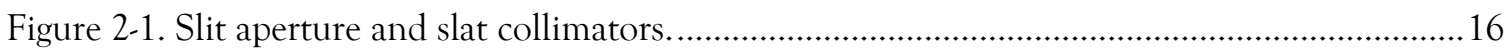

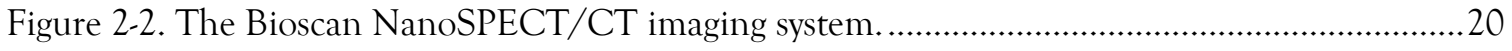

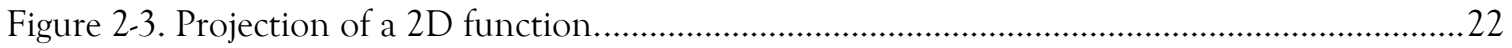

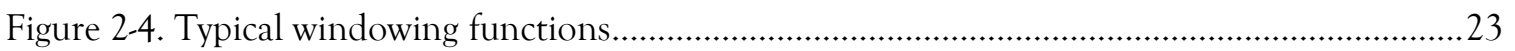

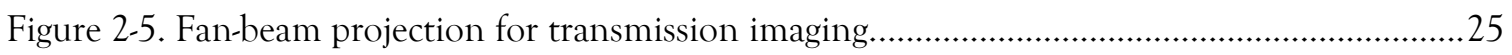

Figure 2-6. Maximum Likelihood-Expectation Maximization(ML-EM) algorithm .............................31

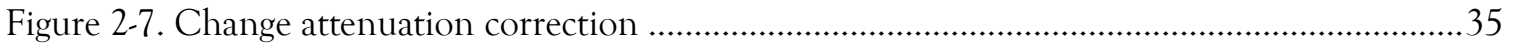

Figure 2-8. Energy windows used for dual energy window scatter correction ..................................... 40

Figure 3-1. Dimensions of the APT2 aperture plate used for rat imaging. ........................................48

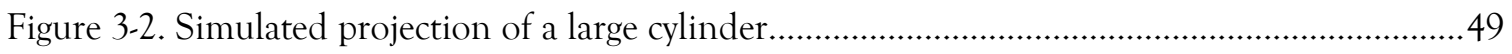

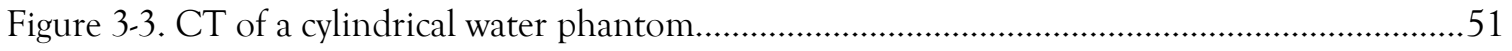

Figure 3-4. The relative intensity of a $45 \mathrm{kV}$ x-ray beam as a function of water thickness ................52

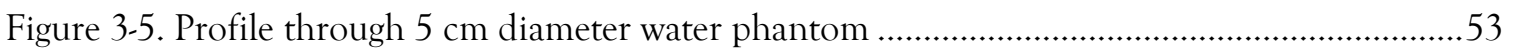

Figure 3-6. Reconstruction of a $50 \mathrm{~mm}$ diameter cylindrical phantom ............................................63

Figure 3-7. Reconstructions of cylindrical digital phantoms ……......................................................65

Figure 3-8. Reconstructions of $9 \mathrm{~mm}, 27 \mathrm{~mm}$, and $37 \mathrm{~mm}$ phantoms containing ${ }^{99 \mathrm{~m}} \mathrm{Tc} \ldots \ldots \ldots \ldots \ldots \ldots . . . . . . . .67$

Figure 3-9. Depth dependence of Capintec CRC-25R dose calibrator ............................................69

Figure 4-1. Rat positioned on animal bed with ECG leads attached to its paws................................73

Figure 4-2. Seventeen segment cardiac model ...............................................................................

Figure 4-3. Volume of the left ventricle of rat 1 plotted against the mass of the rat .........................77 
Figure 4-4. Normal database

Figure 4-5. Z-scores for all five scans of the one of the infarcted rats.

Figure 5-1. Phantoms used for evaluation of absolute activity quantification ..................................97

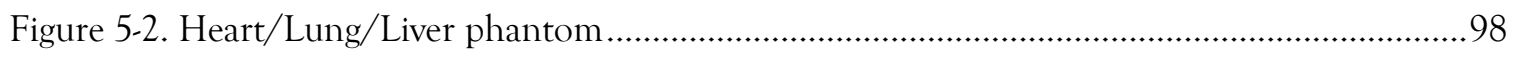

Figure 5-3. Conversion from CT number to linear attenuation coefficients .....................................99

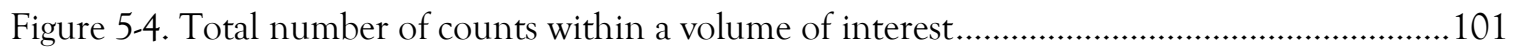

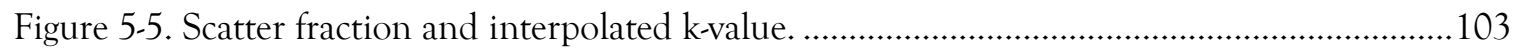

Figure 5-6. Activity concentrations normalized to true value .........................................................104

Figure 6-1. Average of normalized segment perfusion for ${ }^{99 \mathrm{~m}} \mathrm{Tc}$ scans. .......................................118

Figure 6-2. Average of normalized segment perfusion for ${ }^{201} \mathrm{Tl}$ scans. .........................................119

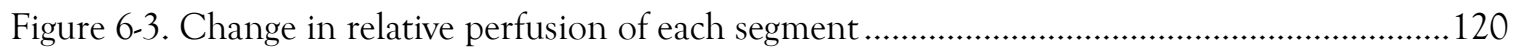

Figure 6-4. Total myocardial uptake as a percentage of injected dose ..............................................123 


\section{Abbreviations and Notation}

COR Centre of rotation

CT Computed tomography, alone it usually refers to X-ray transmission tomography

DEW Dual-energy window, a technique for estimating the scatter contribution in SPECT projections

FBP Filtered backprojection, an analytical algorithm for recovering tomographic images from projections

FDK Feldkamp-Davis-Kress, an approximate filtered backprojection algorithm for reconstructing images from cone-beam projections.

FOV Field of view, the volume of the subject "visible" to the scanner

FWHM Full-width at half maximum, when used to specify resolution, the width of the point spread function at where the intensity is half that of the peak

LAC Linear attenuation coefficient

MMP Multiplexed multi-pinhole, a pinhole collimation scheme that produces multiple overlapping projections on the detector

MR Magnetic resonance

PET Positron emission tomography

PSF Point spread function, the distribution of photon intensities at the detector produced by a point source

ROI Region of interest

SPECT Single photon emission computed tomography

TEW Triple energy window, a technique for estimating the scatter contribution in SPECT projections

VOI Volume of interest 


\section{Introduction}

\section{I.I SPECT}

Single Photon Emission Computed Tomography, or SPECT, is an important tool in the spectrum of medical imaging technologies. Whereas other imaging modalities, X-ray computed tomography (CT) and magnetic resonance imaging (MR) for example, permit non-invasive visualization of anatomy, SPECT and the related imaging modality positron emission tomography (PET), make it possible to visualize physiologic processes in vivo. Such processes include cardiac blood flow, bone creation, renal clearance, or the abundance of a receptor of interest.

Clinically, SPECT is most commonly used in oncology, to locate rapidly growing tumours, and in cardiology to assess myocardial perfusion, cardiac function and tissue viability. Beyond this, SPECT is used for a variety of less common but more specialized diagnostic procedures. For research purposes, SPECT finds application in the development and improvement of the clinical tests already mentioned, as well as in more basic scientific investigation of fundamental biological processes and disease progression. Most pre-clinical and basic science research is done in animal models, principally mice and rats, but also pigs, dogs, rabbits, and variety of other species. 
The underlying principle behind SPECT is simple: a pharmaceutical agent designed to follow a particular biochemical pathway is injected into the subject, either a clinical patient undergoing diagnosis of disease, or a lab animal being used for basic research. Attached to the pharmaceutical is a radioactive isotope that will emit gamma rays when it decays which can be detected from outside the subject. This pharmaceutical-isotope combination is known as a tracer. A camera detects the emitted radiation, allowing the distribution of the pharmaceutical to be observed noninvasively. Computerized reconstruction techniques allow three-dimensional tracer distributions to be extracted from a set of planar projections.

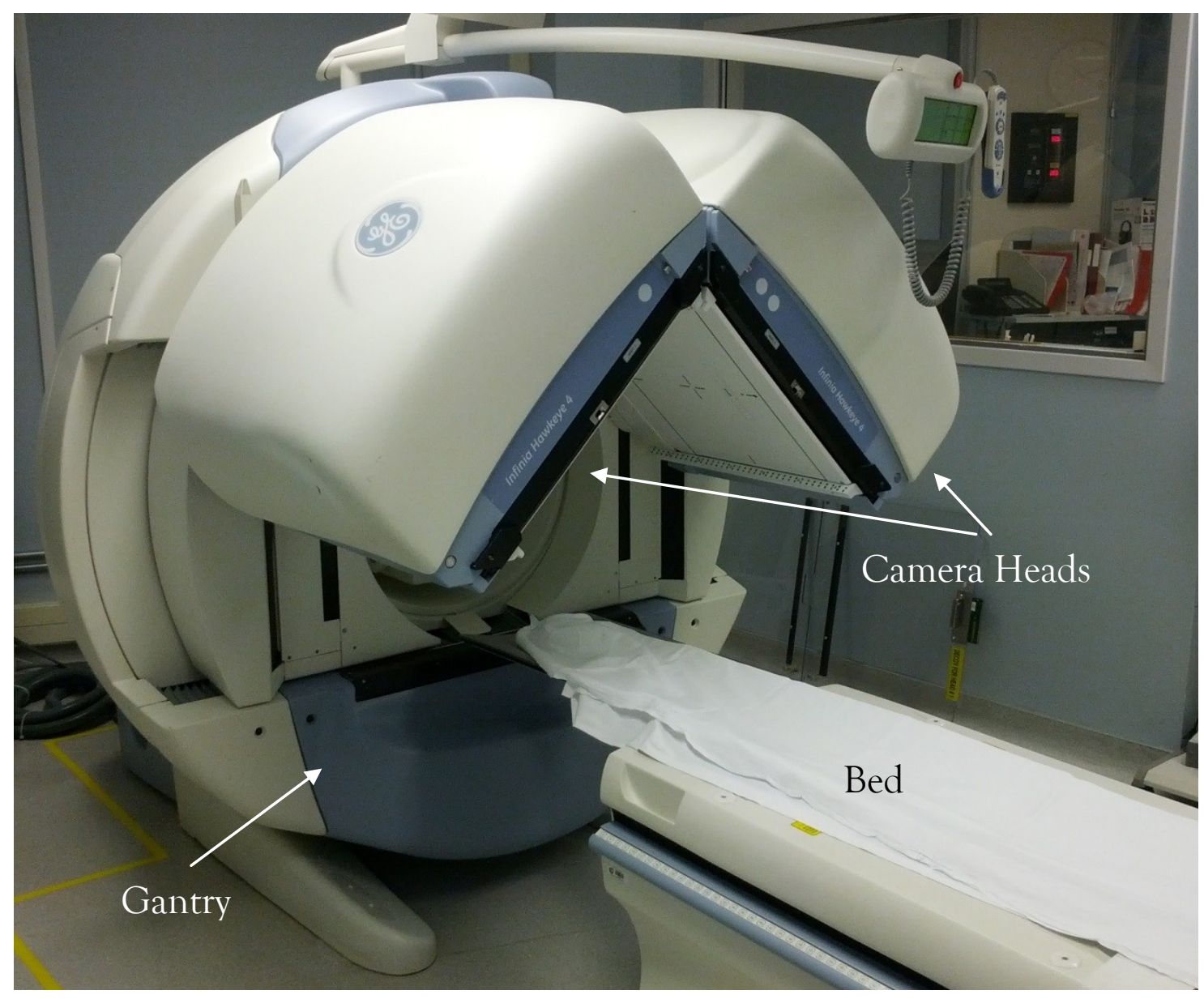

Figure 1-1. Dual-head clinical SPECT camera (Infinia Hawkeye 4, GE Healthcare). 


\section{I.ISPECT basics}

A modern clinical SPECT camera is shown in Figure 1-1. The patient, who has been injected with the tracer, lies on the bed and the gantry rotates around the patient. Once a full set of projection data has been acquired, it can be mathematically reconstructed to create a set of images of transverse slices through the patient. Reconstruction of tomographic images from projection data is discussed in $\$ 2.2$.

\section{I.2 Collimation}

The collimator acts as the lens of the gamma camera. Gamma rays are emitted isotropically from the tracer. Unlike visible light, photons in the $\mathrm{keV}$ range cannot be focused by refraction in the way an optical camera lens focuses visible light. Therefore, absorptive collimation is used to fix the direction of the detected gamma rays (Figure 1-2). A parallel hole collimator, like those used for most clinical SPECT imaging, consists of a thick lead or tungsten sheet with holes which only permit photons entering nearly perpendicular to the collimator to pass. Collimator design is a trade-off between spatial resolution and photon collection efficiency. Collimators with smaller, longer holes provide better resolution, but at the price of reduced efficiency. Alternatively, wider, shorter holes allow more photons entering at a broader range of incident angles to reach the detector, increasing collection efficiency, but degrading the accuracy of directional information and hence spatial resolution.

The parallel-hole collimators used for clinical SPECT imaging typically have resolutions (full width at half maximum (FWHM)) between 7-13 $\mathrm{mm}$ for a point source about $10 \mathrm{~cm}$ from the collimator surface and efficiencies ranging from less than $0.02 \%$ to approximately $0.06 \%$. The Anger camera (Anger, 1958) itself also has a limited intrinsic resolution of approximately 3-4 mm. Together, these limit the optimal spatial resolution of the reconstructed images to no better than $5-10 \mathrm{~mm}$. 
The anatomical structures in small animals have typical dimensions on the order of millimetres, well below the resolution achievable with even high-resolution parallel-hole collimation.

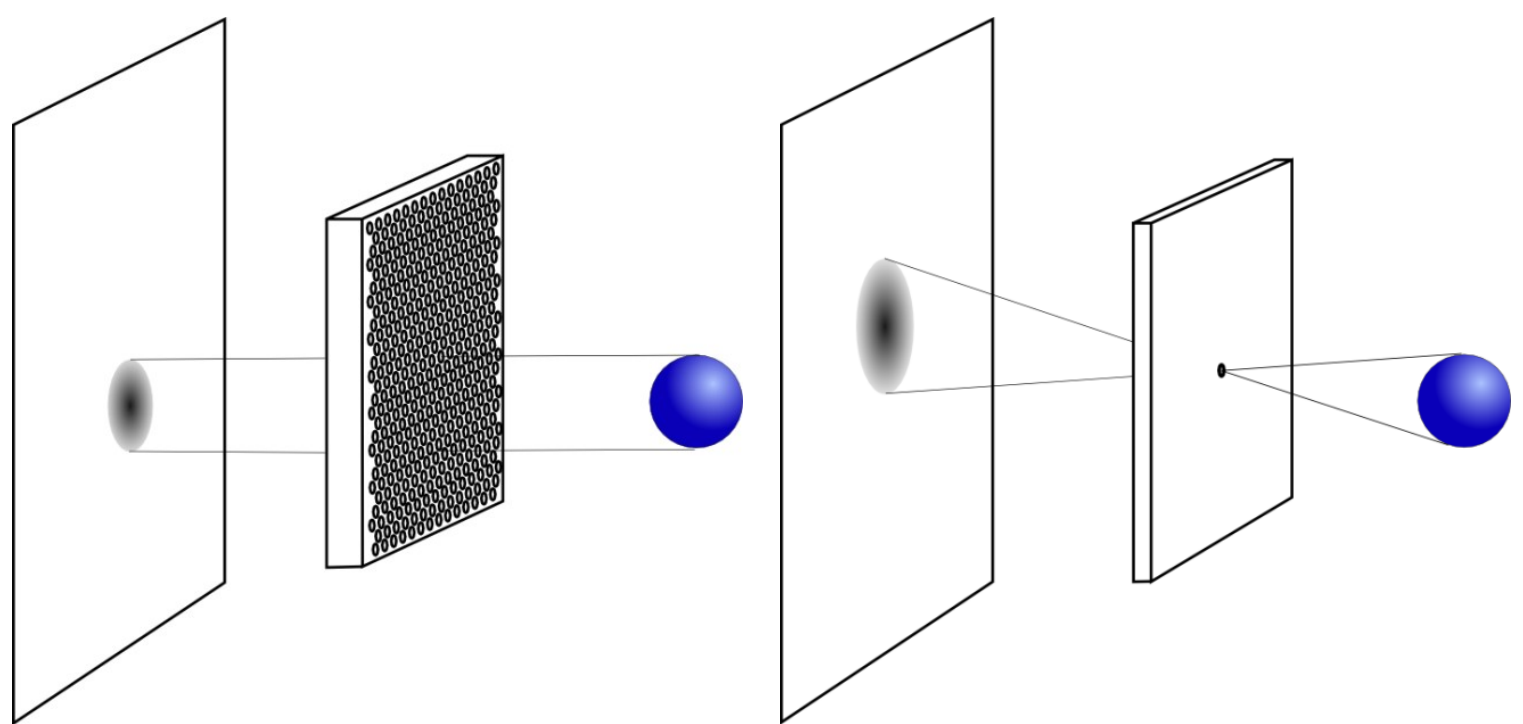

Figure 1-2. Parallel-hole (left) and pinhole (right) collimation. The blue sphere represents the source distribution being projected onto the planar detector.

\section{I.2.I Pinhole collimation}

To overcome these resolution limitations, small animal SPECT imaging employs pinhole collimation (Figure 1-2). The intrinsic magnifying properties of pinhole collimation make it possible to acquire SPECT images with resolutions of less than a millimetre - an order of magnitude better than that achievable with parallel-hole collimation. In exchange for improved resolution, pinhole collimation sacrifices efficiency. Reconstruction of the tracer distribution from pinhole projections is also considerably more complicated than reconstruction from parallel projections. This additional complexity extends as well to any corrections which must be made for accurate reconstruction, including attenuation correction and collimator modelling, discussed in greater detail in a later chapter.

The earliest publication on pinhole SPECT for small animal research is a paper by Palmer \& Wollmer who, using a custom pinhole aperture fitted to a clinical camera, were able to acquire images of phantoms and rabbits with resolutions of $4.4 \mathrm{~mm}$ (Palmer \& Wollmer, 1990). Several 
groups reported on the development of similar pinhole SPECT systems throughout the 1990s. Development of commercial cameras for pre-clinical imaging of laboratory animals followed in the 2000s. A detailed review of these developments follows in $\$ 2.1$.

Pinhole collimation is used in some specialized clinical applications for planar nuclear imaging of the thyroid or the femoral head, but is not used for clinical tomographic imaging with traditional cameras. Some dedicated cardiac SPECT cameras also employ pinhole collimators for minification instead of magnification, allowing a large number of projections to be acquired simultaneously (Bocher, Blevis, Tsukerman, Shrem, Kovalski, \& Volokh, 2010). These new cameras are presently small in numbers but are starting to blur the dividing line between clinical and small-animal camera designs.

\section{I.2.2 Multiplexing of pinhole projections}

Since photons are emitted isotropically but only detected if they pass through the collimator, the intrinsic collection efficiency of pinhole SPECT is proportional to

$$
\frac{d^{2} \cos \theta}{16 r^{2}}
$$

where $d$ is the pinhole diameter, $\theta$ is the angle between the axis of the pinhole and the path from the source to the pinhole, and $r$ is the distance from the source to the pinhole. A pinhole collimator can achieve even better efficiency than a parallel-hole collimator by placing the source very close to the pinhole. However, in a pinhole SPECT configuration, the $\frac{1}{r^{2}}$ falloff in efficiency with source-to-collimator distance leads to large variations in efficiency over the field of view, unlike in a parallel-hole configuration where the sensitivity is approximately uniform. For example, in a rat-imaging configuration, with a collimator radius of rotation of $45 \mathrm{~mm}$, a cylindrical field of view (FOV) with a radius of $25 \mathrm{~mm}$ (FOV: $20 \mathrm{~mm}<r<70 \mathrm{~mm}$ ), and a pinhole diameter of $2 \mathrm{~mm}$, 
the collimator efficiency for a source along the pinhole axis is approximately $0.06 \%$ (better than with a high-sensitivity parallel-hole collimator) at the edge of the FOV closest to the pinhole and falls off as $\frac{1}{r^{2}}$ to $0.005 \%$ at the far edge. Efficiency is even lower away from the pinhole axis.

The problem of low efficiency is particularly acute in small animal SPECT imaging because, to achieve levels of statistical accuracy comparable to those in clinical SPECT, the total number of counts recorded for each voxel of the subject must be similar. However, the voxels used by microSPECT are several orders of magnitude smaller than those used clinically. Whereas a clinical image might be reconstructed on a grid of voxels $5 \mathrm{~mm}$ or more on each side, the voxels in small animal images can be less than $1 \mathrm{~mm}$ per side. The volumes of the voxels used in small animal SPECT are less than $1 / 100^{\text {th }}$ (and possibly as small as $1 / 1000^{\text {th }}$ ) of those used in clinical SPECT. This implies that the tracer concentration, the scan duration, the collimator efficiency, or some combination of the three must be increased commensurately to compensate. Therefore, most small animal scanners incorporate multiple heads.

Collection efficiency can be further improved with multiple pinholes on each detector. However, in this arrangement, the potential exists for projections to overlap on the detector, making it unclear through which pinhole a detected photon came. One possibility for removing the ambiguity is to arrange the pinholes and the magnification to eliminate any overlap. Unfortunately, this compromises the magnification and therefore the resolution of the final image. It also tends to leave unused regions on the detector surface that fall outside the field of view of all of the pinholes. The other possibility is to multiplex the projections; allowing them to overlap. This makes optimal use of the available detector surface and allows for greater magnification of the projections than the un-multiplexed approach, but introduces additional challenges in the reconstruction, and 
potentially creates irresolvable ambiguities when reconstructing projection data with too much overlap.

More detailed discussion of the tradeoffs between resolution, efficiency, optimal detector usage, and the challenge of unambiguous reconstruction follows in $\$ 3.5$.

\section{I.3 Isotopes}

SPECT imaging is performed with isotopes that emit a gamma ray when they decay. Various criteria determine the suitability of an isotope for SPECT imaging: the energy of the photon emitted when it decays, the half-life of the isotope, its chemical properties, and its availability. For clinical scans, usable isotopes are limited to those with energies of at least $70-80 \mathrm{keV}$, though for small-animal research the reduced attenuation makes it possible to use lower energy isotopes, down to about $25 \mathrm{keV}$.

Table 1-1. Commonly used SPECT isotopes.

\begin{tabular}{|c|c|c|c|}
\hline Isotope & "Gamma energy & Half-life & Notes \\
\hline${ }^{67} \mathrm{Ga}$ & $\begin{array}{l}93 \mathrm{keV}, 185 \mathrm{keV}, \\
300 \mathrm{keV}\end{array}$ & 3.26 days & $\begin{array}{l}\text { Free } \mathrm{Ga}^{3+} \text { ion acts as a tracer for inflammation and } \\
\text { tumour growth. }\left({ }^{68} \mathrm{Ga} \text { is a positron emitter that can be }\right. \\
\text { used to image the same processes using PET.) }\end{array}$ \\
\hline${ }^{99 \mathrm{~m}} \mathrm{Tc}$ & $140.5 \mathrm{keV}$ & $6 \mathrm{hrs}$ & $\begin{array}{l}{ }^{99 \mathrm{~m}} \mathrm{Tc} \text { csestamibi for myocardial perfusion imaging } \\
{ }^{99 \mathrm{~m}} \mathrm{Tc} \text {-tetrofosmin also used for myocardial perfusion } \\
{ }^{99 \mathrm{~m}} \mathrm{Tc} \text {-methylene diphosphonate (MDP) for bone scans }\end{array}$ \\
\hline${ }^{111}$ In & $171 \mathrm{keV}, 245 \mathrm{keV}$ & 2.8 days & $\begin{array}{l}{ }^{111} \text { In -octreotide for neuroendocrine tumour imaging } \\
{ }^{111} \text { In-oxime for white blood cell infection studies }\end{array}$ \\
\hline${ }^{123} \mathrm{I}$ & $159 \mathrm{keV}$ & $13.3 \mathrm{hrs}$ & $\begin{array}{l}{ }^{123} \text { I-iobenguane (MIBG) for imaging sympathetic } \\
\text { innervations in the heart, neuroendocrine tumours } \\
{ }^{123} \text { I-ioflupane (DaTSCAN) for assessing dementia }\end{array}$ \\
\hline${ }^{131} \mathrm{I}$ & $364 \mathrm{keV}, 636 \mathrm{keV}$ & 8 days & $\begin{array}{l}\text { Used for therapy and diagnosis of thyroid carcinoma, } \\
\text { poor resolution for imaging }\end{array}$ \\
\hline${ }^{201} \mathrm{Tl}$ & $68 \mathrm{keV}-83 \mathrm{keV}$ & $72.9 \mathrm{hrs}$ & $\begin{array}{l}\text { Cardiac perfusion tracer, more commonly used before } \\
\text { the development of }{ }^{99 \mathrm{~m}} \mathrm{Tc} \text { based tracers. The photons } \\
\text { used for imaging are the characteristic x-rays of } \mathrm{Hg} \text {. }\end{array}$ \\
\hline
\end{tabular}


The quality of SPECT images depends on the number of photons detected during a scan, which depends on the total amount of activity administered, the isotope half-life, the efficiency of the camera, and the duration of the scan. For clinical applications, it is also desirable that the half-life of the tracer be short to reduce the total dose absorbed by the patient. Isotopes frequently used for SPECT imaging are listed in Table 1-1, with their energies and half lives.

\section{I.4 Basic research and pre-clinical imaging}

In addition to clinical applications, SPECT also plays an important role in pre-clinical research. Thanks to the genetic similarities between humans and small animals, animal models of various human diseases can be developed, and have been developed for both mice and rats. Before being used clinically, both diagnostic and therapeutic pharmaceuticals are almost universally tested in small animal models. Promising candidates are then further developed in larger animals and possibly primates before eventually being translated into human use.

Pinhole SPECT systems designed for preclinical imaging of mouse and rat models can provide images of tracer distributions with sub-millimetre resolution (Meikle, et al., 2002) (Franc, Acton, Mari, \& Hasegawa, 2008) (Nuyts, Vunckx, Defrise, \& Vanhove, 2009). Such microSPECT systems are a valuable tool for researching disease progression and treatment efficacy in small animal models of human diseases. The work in this thesis is devoted to cardiac imaging in rats. Various rat models of cardiovascular diseases and modes of heart failure have been developed over the past decades (Hasenfuss, 1998). With ECG gating, high-resolution small-animal SPECT cameras can be used to measure aspects of cardiac function including myocardial perfusion, left ventricle (LV) volumes and ejection fraction in rats. Figure 1-3 shows a SPECT image of a rat injected with a myocardial perfusion tracer. 
MicroSPECT measurements are subject to various uncertainties. Some of these arise from the nature of SPECT itself, such as statistical noise. Other uncertainties are associated with the subject being imaged, for example, the challenge of maintaining a constant physiological state in a live animal over an appreciable length of time. Finally, additional uncertainty may be introduced by the techniques used to analyze the images and extract relevant measurements. Understanding the magnitude of these uncertainties is essential any time microSPECT is used to measure and compare cardiac parameters for example when evaluating changes from disease progression, intervention, or the introduction of new technology or scanning techniques.

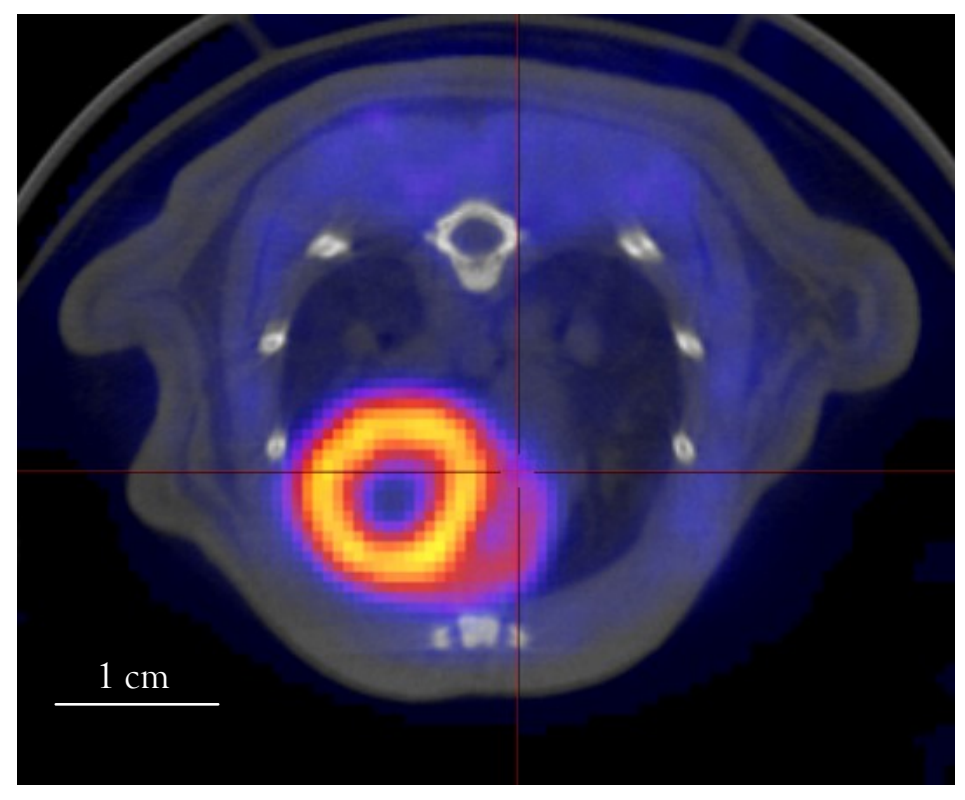

Figure 1-3. Trans-axial SPECT image of the thorax of rat injected with $99 \mathrm{mTc}$-tetrofosmin overlaid on a CT scan (grey scale). SPECT voxels have dimensions of $(0.6 \mathrm{~mm})^{3}$. (The resolution of the SPECT images is $\sim 1.9 \mathrm{~mm}$ )

To be useful as a tool for basic research, it is desirable that the measurements made with SPECT be both quantitatively accurate and reproducible. Furthermore, several important differences distinguish small animal SPECT from clinical SPECT and make it necessary to revisit the problem of obtaining quantitative SPECT data, a problem that has been extensively researched for clinical SPECT over the past 30-40 years. The primary factors limiting the quantitative accuracy of SPECT 
are photon attenuation and scatter (Jaszczak, Coleman, \& Whitehead, 1981), collimator dependant resolution effects, and partial volume effects.

Reproducibility is also essential in quantitative medical imaging. Although as with any measurement, and especially those involving biological systems, some variation is inevitable, minimizing the contribution of the imaging system itself to the variance makes it possible to make more accurate measurements with smaller sample sizes, increasing accuracy while reducing the costs associated with basic research.

\section{I.5 Approach and outline of thesis}

The focus of this thesis is to evaluate the quantitative accuracy and reproducibility of small-animal cardiac SPECT data. Initial evaluation of the reproducibility of rat cardiac data was done using the manufacturer reconstruction software. Custom software which models attenuation and compensates for scatter as part of reconstruction was then developed and used to investigate the quantitative accuracy of measurements made using SPECT scans of phantoms and in rats.

Chapter 2 is a review of the existing literature describing the development of high-resolution small animal SPECT imaging systems, the various approaches taken to addressing attenuation and scatter correction in clinical systems, and how these approaches have been adapted to small animal imaging systems. The still relatively limited literature dedicated to quantification in small animal SPECT imaging is also summarized.

Chapter 3 details the hardware and software used in this research. The nanoSPECT/CT camera from Bioscan (Washington, DC) will be described along with the relevant acquisition parameters used for our experiments. Chapter 3 also outlines the custom SPECT and CT reconstruction software developed for this work. The hardware vendor does provide software for reconstruction, which was used for investigation of reproducibility in Chapter 4. However the vendor 
reconstruction code HiSPECT (SciVis, Gottingen, Germany), is proprietary and locked, making it impossible to modify the algorithm to correct for attenuation or scatter within the reconstruction. Chapter 3 concludes with some simulation data investigating the limits of the nanoSPECT imaging geometry and some measurements necessary for the work in the following chapters, but not directly relevant to the topic of the thesis.

In Chapter 4 the reproducibility of cardiac SPECT measurements in rats is evaluated. This comprised original research on the reproducibility of rat left ventricle volumes (systolic and diastolic), ejection fraction, and myocardial perfusion. This study included both normal rats and rats with a surgically induced myocardial infarction. The results of this work were published in the Journal of Nuclear Medicine (Strydhorst, Leenen, Ruddy, \& Wells, 2011).

Then in Chapter 5, the quantitatively accuracy of SPECT measurements made with data acquired using the NanoSPECT/CT system is assessed. Photon attenuation and scatter are modelled as part of the image reconstruction algorithm and the effect on SPECT quantification is assessed. The methods employed for scatter and attenuation correction are modifications of techniques already well established for clinical SPECT. The application of these methods to a multiplexing, multipinhole small animal imaging system, and an analysis of their efficacy for obtaining quantitative SPECT data with such a system has not previously been published.

In Chapter 6, the reproducibility measurements done in Chapter 4 are repeated, using the reconstruction code developed in the work reported in Chapter 5. Improving the quantitative accuracy may influence the reproducibility of the measurements because incorporating additional information into the reconstruction adds additional noise and potential sources of error. The impact of the corrections on reproducibility are assessed and it is concluded that reproducibility is not degraded by attenuation and scatter correction. 
Chapter 7 summarizes the results and proposes some possible avenues of further research. 


\section{Background and Literature Review}

\section{I Development of pre-clinical SPECT imaging systems}

\section{I.I Experimental/Custom systems}

The earliest published work on small animal pinhole SPECT is (Palmer \& Wollmer, 1990). Their camera, like much of the other early experimental work on pinhole SPECT, was created by fitting pinhole collimators to clinical cameras. With a single $3.3 \mathrm{~mm}$ aperture pinhole, they were able to image phantoms containing ${ }^{99 \mathrm{~m}} \mathrm{Tc}$ and ${ }^{133} \mathrm{Xe}$ gas, and rabbit lungs using ${ }^{99 \mathrm{~m}} \mathrm{Tc}-$ labed aerosol particles. Acquiring the projection data for the rabbit lungs took $60 \mathrm{~min}$, and the reconstructed images had a resolution of $4.4 \mathrm{~mm}$.

Several groups, including (Weber, et al., 1994) (Jaszczak, Li, Wang, Zalutsky, \& Coleman, 1994), (Ishizu, et al., 1995) and (Ogawa, Kawade, Nakamura, Kubo, \& Ichihara, 1998), have experimented with various pinhole SPECT systems, characterising the relationships between pinhole aperture size, radius of rotation, and focal length (detector to aperture distance), and achievable resolution and efficiency. Using a $2.0 \mathrm{~mm}$ pinhole Weber et al. acquired ${ }^{201} \mathrm{Tl}$ and ${ }^{99 \mathrm{~m}} \mathrm{Tc}$ images of normal rat brains, brain tumours, and hearts, with a resolution of $2.8 \mathrm{~mm}$. Jaszczak et al. mounted a custom pinhole apparatus on one of the cameras of a triple-head clinical SPECT camera, with which they 
were able to acquire images of comparable resolution: $1.1-2.0 \mathrm{~mm}$ with a $1.0 \mathrm{~mm}$ pinhole; $2.5-3.6$ $\mathrm{mm}$ with a $2.4 \mathrm{~mm}$ pinhole. Using their system they acquired ${ }^{99 \mathrm{~m}} \mathrm{Tc}$ bone and liver scans of rats. Similar work by Ishizu et al. using a four-head clinical camera was able to achieve resolutions of $1.65 \mathrm{~mm}$ (1.0 $\mathrm{mm}$ aperture). They also demonstrated rat heart imaging with ${ }^{99 \mathrm{~m}} \mathrm{Tc}$, and rat brain imaging using ${ }^{123} \mathrm{I}$. The Ogawa system used a $1.0 \mathrm{~mm}$ pinhole and a slightly longer focal length to achieve ${ }^{99 \mathrm{~m}} \mathrm{Tc}$ images of rat myocardia with a resolution of $1.1 \mathrm{~mm}$. Myocardial infarction in rats was first imaged by (Yukihiro, Inoue, Iwasaki, Tomiyoshi, Erlandsson, \& Endo, 1996), who used a $2.0 \mathrm{~mm}$ and $3.3 \mathrm{~mm}$ diameter apertures fitted to a clinical system to acquire ${ }^{201} \mathrm{Tl}$ images.

For most of the early research into small-animal pinhole SPECT systems, the images were reconstructed using a modified cone-beam filtered backprojection reconstruction algorithm (see §2.2.1.1). Jaszczak et al. also reconstructed some of their phantom scans using a maximum likelihood (ML-EM) algorithm, which significantly reduced axial distortion in the reconstruction relative to the FBP reconstruction, but required over 60 hours to reconstruct in 1994, compared to less than an hour for the FBP algorithm.

In addition to their bulk, clinical systems with pinhole collimators can exhibit center-of-rotation $(\mathrm{COR})$ shifts that are large relative to the desired resolution of the acquired image. If the COR error is known, it can be compensated for in the reconstruction algorithm (Jaszczak, Li, Wang, Zalutsky, \& Coleman, 1994). Partly in an attempt to improve the mechanical precision of the scanner for pre-clinical research applications, several groups started building dedicated small-animal systems. Some of these used a stationary camera and a rotating animal holder (Wu, et al., 2000), reminiscent of early clinical SPECT systems which placed the patient on a rotating chair. To minimize shifting of the subject during acquisition, the animal tube is rotated around a vertical 
axis. With such a custom built system and $0.5 \mathrm{~mm}$ pinholes $\mathrm{Wu}$, et al. were able to acquire gated images of mouse myocardia with a resolution of approximately $1.0 \mathrm{~mm}$.

Sub-millimetre resolution tomographic imaging of living mice was achieved by (McElroy, et al., 2002). With the A-SPECT pinhole SPECT camera (Gamma Medica, Inc. Northridge, CA), consisting of a pinhole camera mounted in front of a rotating tube they were able to acquire images with an axial spatial resolution as low as $0.53 \mathrm{~mm}$. Planar images of mouse thyroids with resolutions as high as $200 \mu \mathrm{m}$ have also been acquired using micro-pinholes (100 $\mu \mathrm{m}$ in diameter) and ${ }^{125} \mathrm{I}$ as the isotope (Beekman, McElroy, Berger, Gambhir, Hoffman, \& Cherry, 2002). In the latter case, the long acquisition time, $50 \mathrm{~min}$ for a single planar image, is probably prohibitive for in vivo tomographic imaging.

In the case of every single-pinhole imaging system discussed so far, the efficiency was below 100 counts/s per MBq of injected activity, or less than $0.01 \%$. To improve the efficiency of small animal imaging systems, various investigators experimented with forms of coded aperture imaging. Coded aperture imaging is a method for obtaining images of high-energy photons which cannot readily be focused by refraction or reflection. Compared to pinhole collimation, coded-aperture imaging can offer sensitivities several orders of magnitude greater. In coded aperture imaging, the $\mathrm{x}$ rays or gamma rays pass though a plate with multiple zones which are either transparent or opaque to the incident radiation. The result is a complex shadow on a detector behind the aperture plate consisting of multiple overlapping images of the source. Provided certain conditions are met, the shadow image can be de-convolved to obtain the source distribution.

Coded aperture imaging was originally developed for x-ray astronomy, and its use for nuclear medicine was investigated as early as 1972 (Barrett, 1972). Clinically, coded aperture imaging has been implemented in various systems using rotating slit apertures or slat collimators (Figure 2-1) 
(Gindi, et al., 1982). With slit or slat aperture collimation, activity in the plane defined by the aperture and a stripe on the detector parallel to the aperture contributes to the activity detected along that stripe. By rotating the aperture through a range of $\pi$ radians, sufficient data are obtained to reconstruct a $2 \mathrm{D}$ projection of the source object. (In the case of the slit collimator, the scaling of the projection of each plane depends on depth.) Full tomographic reconstruction also requires that the detector be moved around the object (in addition to a $\pi$ radians rotation at each projection angle) to sample a full set of planes. Either slit or slat aperture collimation alone offers significant collection efficiency gains over parallel hole and pinhole collimation, though this is partly offset by the need collect multiple projections to reconstruct each planar image. Slit collimation, like pinhole collimation, also magnifies the projections (provided the detector-collimator distance is greater than the object-collimator distance).

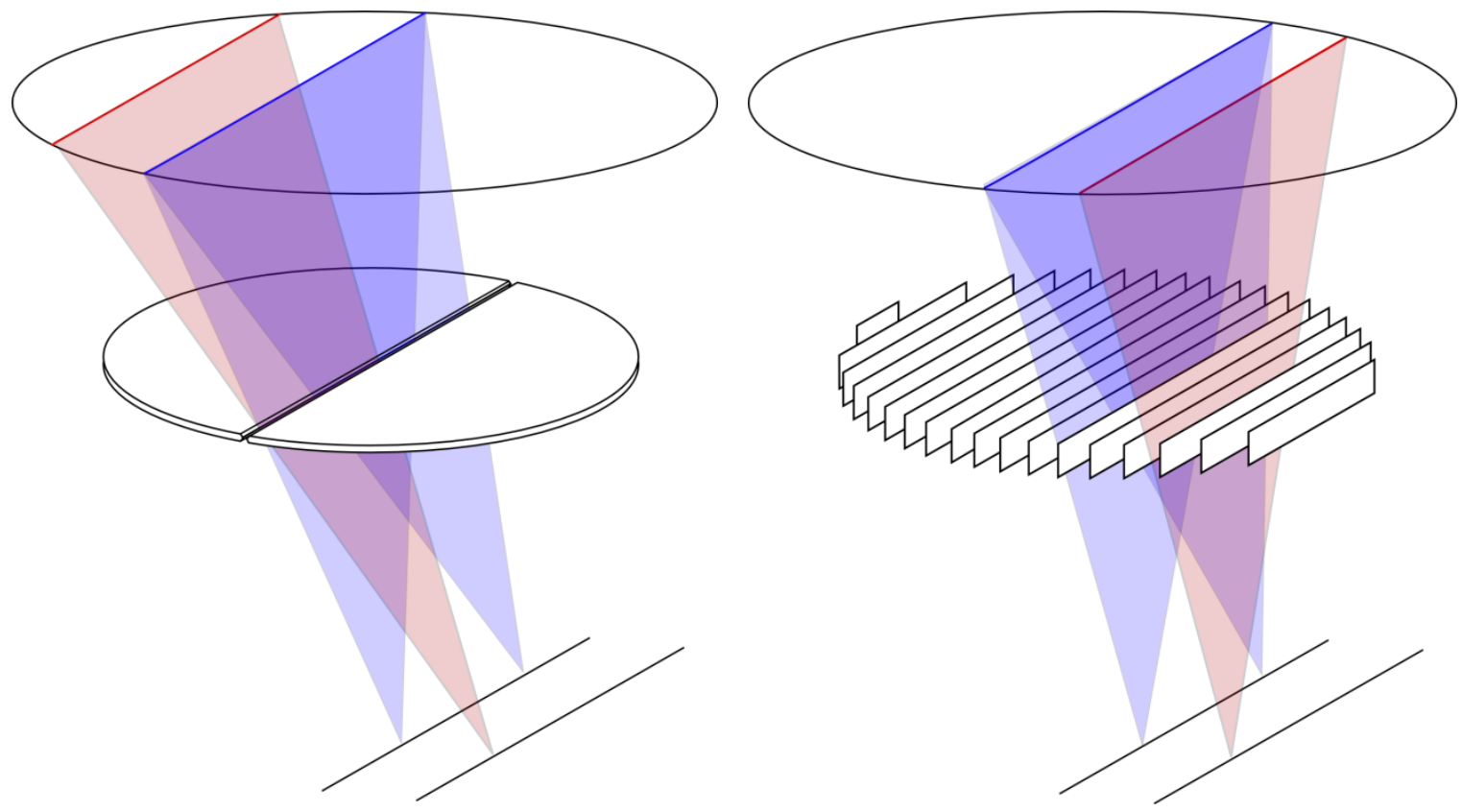

Figure 2-1. Slit aperture (left) and slat (right) collimators.

Combined slit-slat collimation, with the slit parallel to the axis of the scanner and the slats perpendicular to the slit, results in a fan-beam imaging geometry. In this case, the efficiency will be 
somewhere between that of parallel-hole and that of pinhole collimation, and magnification is confined to the trans-axial direction; resolution in the axial dimension will be limited by the slat collimation and the intrinsic resolution of the detector. In exchange for the loss of axial resolution, the slit-slat collimator typically permits a longer axial field of view than a pinhole. Cameras capable of full-body mouse imaging have been developed by (Walrand, Jamar, de Jong, \& Pauwels, 2005), and (Cao \& Peter, 2012). The scanner of Walrand et al. also used linear orbit acquisition: instead of rotating along a circular path around the subject, four detectors move linearly, each following one side of the smallest rectangle enclosing the subject. The linear orbit permits the camera to be positioned closer to the subject (improving efficiency), while avoiding truncation problems. Walrand et al. achieved resolutions better than $0.65 \mathrm{~mm}$ in the trans-axial plane. Cao \& Peter report a resolution of approximately $1.0 \mathrm{~mm}$ (trans-axial) by about $1.8 \mathrm{~mm}$ (axial).

Multiple-pinhole imaging is a type of coded-aperture imaging and was first tested for clinical SPECT by (Vogel, Krich, LeFree, \& Steele, 1978) in the form of a seven-pinhole collimator with non-overlapping projections. The aim of multi-pinhole collimation was to achieve greater efficiency than is possible with parallel-hole or single pinhole collimation.

Application of coded aperture imaging to small animal SPECT first appeared in work by (Meikle, Fulton, Eberl, Dahlbom, Wong, \& Fulham, 2001). Using simulations, they calculated that they could achieve sensitivities greater than $1 \%$ using random pinhole arrays of up to 100 pinholes, while still achieving spatial resolutions $<2 \mathrm{~mm}$. Preliminary investigations of a prototype multipinhole systems demonstrated the feasibility of multiplexed multi-pinhole small animal imaging systems (Meikle, et al., 2002). With multiple pinholes the efficiency of the system was increased and multiplexing, while it had no effect on the resolution achievable compared to non-multiplexed data, did reduce the signal-to-noise ratio (SNR) of the reconstructed image, and required more 
iterations for an iterative reconstruction algorithm to converge. Similar work by (Schramm, Ebel, Engeland, Schurrat, Béhé, \& Behr, 2003) describes another prototype micro SPECT system, also using multiplexed projections, and employing tilted pinholes arranged to optimize the homogeneity of the sampling of the field of view. (The latter system developed into the nanoSPECT system from Bioscan.)

Instead of multiple pinholes, the FastSPECT II system used multiple cameras, 16 of them, each with a single pinhole arranged in two adjacent rings of eight cameras each around the subject (Furenlid, et al., 2004). An updated version, the FastSPECT III designed specifically for rodent brain imaging, uses 20 cameras (Miller, Furenlid, Moore, Barber, Nagarkar, \& Barrett, 2009).

A design for a small animal SPECT system consisting of a cylindrical multi-pinhole collimator surrounded by a multiple rings of modular detectors was published by (Funk, Després, Barber, Shah, \& Hasegawa, 2006). Simulations predicted a resolution of $0.8 \mathrm{~mm}$ with an efficiency of $630 \mathrm{cps} / \mathrm{MBq}(0.06 \%)$

\section{I.2 Commercial small animal systems}

Within the last decade, several commercial small animal SPECT scanners have been introduced to the market.

The X-SPECT system (Gamma Medica-Ideas, Northridge, CA, USA) - now part of the Triumph trimodality pre-clinical imaging system - featured a dual headed camera with an $83 \times 83 \mathrm{NaI}$ detector matrix of $1.5 \mathrm{~mm} \times 1.5 \mathrm{~mm}$ pixels, a focal length of $9 \mathrm{~cm}$, and interchangeable singlepinhole apertures. The X-SPECT system also features an integrated CT scanner which permits the acquisition of co-registered anatomical information and permits CT-based attenuation correction. 
Unlike most SPECT scanning systems where the camera heads are rotated around the subject to acquire sufficient angular projection data for image reconstruction, the U-SPECT-I system (Beekman, et al., 2006) and its successor, U-SPECT-II (MILabs, Utrecht, The Netherlands) uses three stationary camera heads (van der Have, et al., 2009). A full range of angular projection data is acquired by the spacing of a set of 75 focussing pinholes drilled in a cylindrical collimator surrounding the subject. The pinholes are oriented to focus on a common field of view in the centre of the camera and to prevent overlap of the projections on the detector. The scanner is capable of acquiring very high resolution images $(\sim 0.3 \mathrm{~mm}$ resolution with the mouse collimator, $\sim 0.8 \mathrm{~mm}$ with the rat collimator), and avoids the problem of inconsistent projection data when the activity distribution changes during the course of the scan, since all views are acquired simultaneously. The scanner field of view is limited to a diameter of $27 \mathrm{~mm}$ and an axial length of $11 \mathrm{~mm}$ (with the rat collimator) for a single bed position. Multiple bed positions are necessary to acquire images of organs or activity distributions which extend beyond the limited FOV.

The eXplore speCZT (GE Healthcare), was equipped with a circular ring of 10 detectors and interchangeable pinhole and slit-slat collimators allowing acquisition of high resolution $(1 \mathrm{~mm})$ or large FOV (full-body mouse) imaging. The camera also included an integrated CT scanner to acquire co-registered CT images. ${ }^{1}$

The work which follows in this thesis was done with the nanoSPECT/CT small animal imaging system (Bioscan, Washington, DC, USA). The nanoSPECT system comprises four NaI scintillation cameras at every $90^{\circ}$ around the subject (Figure 2-2). To increase the efficiency of the camera and optimize the usage of the available detector area, the multi-pinhole collimators used by the

\footnotetext{
${ }^{1}$ The eXplore speCZT seems not to have been a commercial success. The description given has been assembled from information on the websites of the few institutions which own such a system.
} 
nanoSPECT camera employ a multiplexing scheme such that projections of an extended source distribution partially overlap on the detectors. Interchangeable apertures allow the system to be used for imaging mice or rats with a range of resolutions and sensitivities. Larger animals (rabbits, lizards, marmosets) can be imaged by replacing the pinhole collimators with parallel-hole collimators. A more detailed description of the nanoSPECT/CT hardware and the pinhole multiplexing scheme for the high-efficiency rat apertures is included in the next chapter.

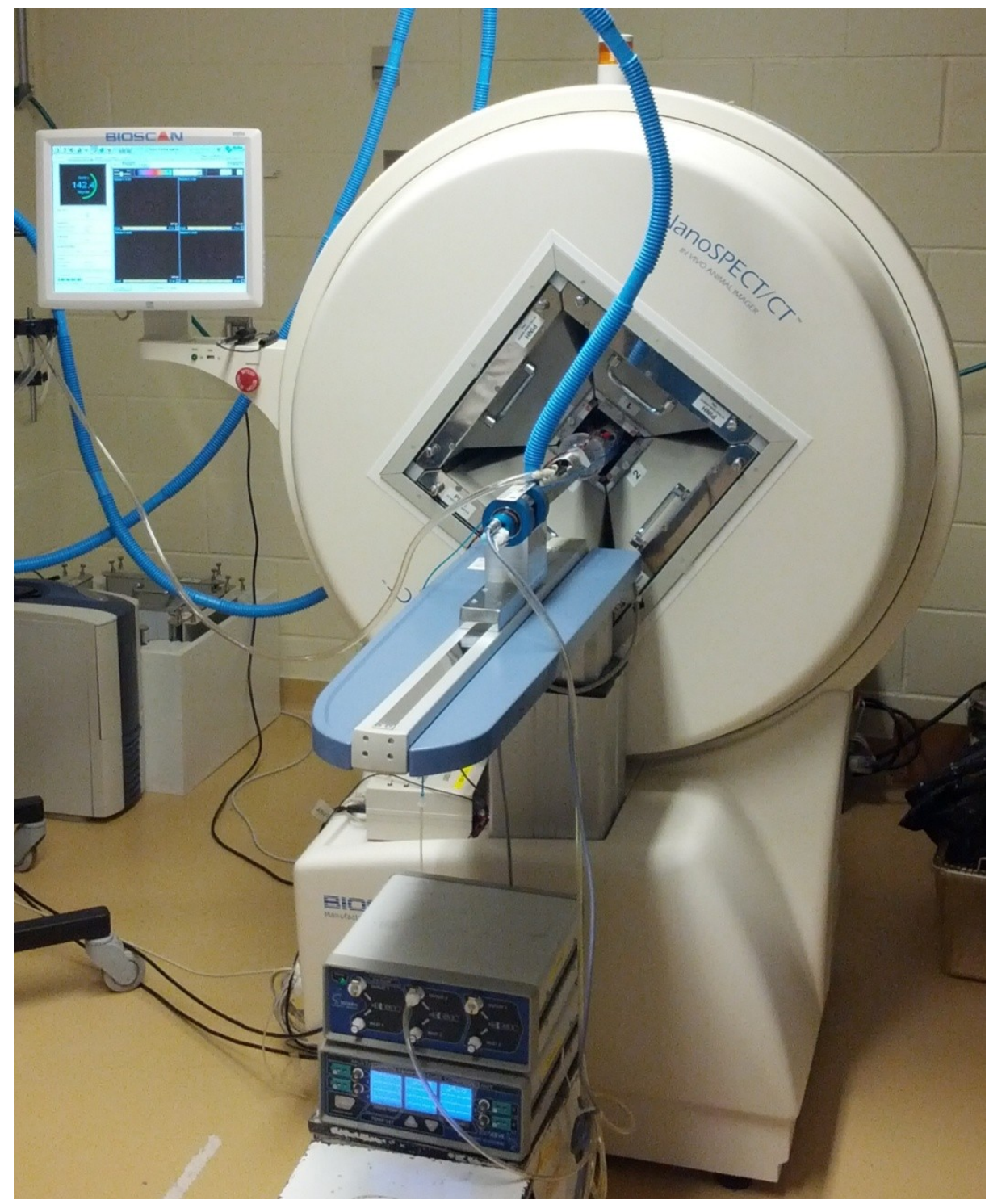

Figure 2-2. The Bioscan NanoSPECT/CT imaging system at the University of Ottawa Heart Institute. 


\subsection{Acquisition and reconstruction of projection data}

In both transmission and emission computed tomography, (CT, PET, and SPECT) the data acquired by the scanner comprises (ideally) a set of 'projections', summations of some quantity along a set of lines intersecting the subject. In transmission computed tomography (CT), the projections are the cumulative linear attenuation along each line; in emission tomography, PET and SPECT, the projection data are the integrated activity along each line.

For the 2D case, shown in Figure 2-3, projection of a continuous function, $f(\boldsymbol{x})$, produces a set of projections, $p_{\phi}(r)$.

$$
p_{\phi}(r)=\int_{-\infty}^{\infty} f(r \boldsymbol{\alpha}+s \boldsymbol{\beta}) d s
$$

where $r$ is the radial distance from the origin parallel to the detector, $s$ is the distance from the origin perpendicular to the detector, and $\boldsymbol{\alpha}$ and $\boldsymbol{\beta}$ are unit vectors parallel and perpendicular to the detector, respectively:

$$
\begin{aligned}
& \boldsymbol{\alpha}=(\cos \phi, \sin \phi) \\
& \boldsymbol{\beta}=(-\sin \phi, \cos \phi)
\end{aligned}
$$

Extension to three dimensions is done by simply collating a set of adjacent 2-D slices along the axis $\mathrm{x}_{3}$ which is perpendicular to the plane $\mathrm{x}_{1}-\mathrm{x}_{2}$.

\subsection{Analytic reconstruction: filtered back-projection}

The mathematics of filtered back-projection (FBP) reconstruction is well established and covered in detail by numerous textbooks. A very brief summary follows, but a more thorough treatment of reconstruction by filtered back-projection can be found in (Kak \& Slaney, 1988). 


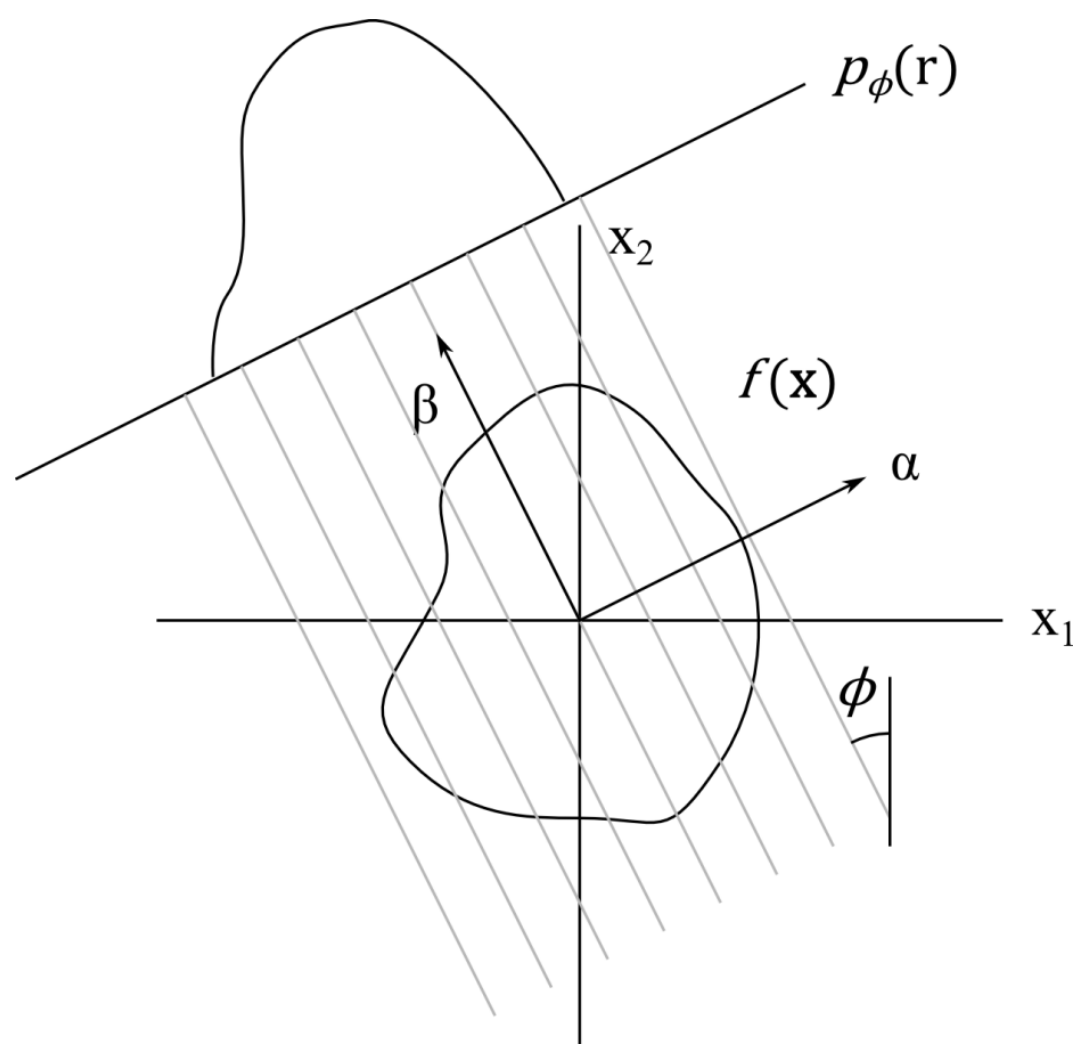

Figure 2-3. Projection of a 2D function.

For the case of a set of line integrals through a 2D function (and the more general case of a set of (N-1)-dimensional integrals through an $\mathrm{N}$-dimensional function, i.e. plane integrals of a function in $\mathbb{R}^{3}$, etc.), Radon had already derived an exact inversion formula as early as 1917 . Given a full set of projections, $p_{\phi}(r)$, where $0 \leq \phi<\pi$, and provided $f(\mathbf{x})$ goes to 0 outside some finite domain:

$$
f(\boldsymbol{x})=\int_{0}^{\pi} \int_{-\infty}^{\infty} P_{\phi}(\rho)|\rho| e^{2 \pi i x \cdot \rho \boldsymbol{\alpha}} d \rho d \phi
$$

Where $P_{\phi}(\rho)$ is the Fourier transform of $p_{\phi}(r)$ :

$$
P_{\phi}(\rho)=\int_{-\infty}^{\infty} p_{\phi}(r) e^{-2 \pi i r \rho} d r
$$

Qualitatively, the algorithm involves filtering each projection in frequency space by a ramp function, then projecting the filtered projection back into the reconstruction domain, and summing the back-projections from every angle. For the actual implementation, the projections and 
reconstruction are discretized: only a finite number of projection angles can be acquired, the pixels that make up the detector are discrete, and the grid on which the image is reconstructed is likewise discrete.

The ramp filter in filtered back-projection emphasizes the high frequencies in projection space, frequencies where noise in the projection data is likely to exceed the signal. In most clinical implementations of FBP, the high frequencies are rolled off by a windowing function, such as a Shepp-Logan, Hann, or Butterworth filter (Figure 2-4).

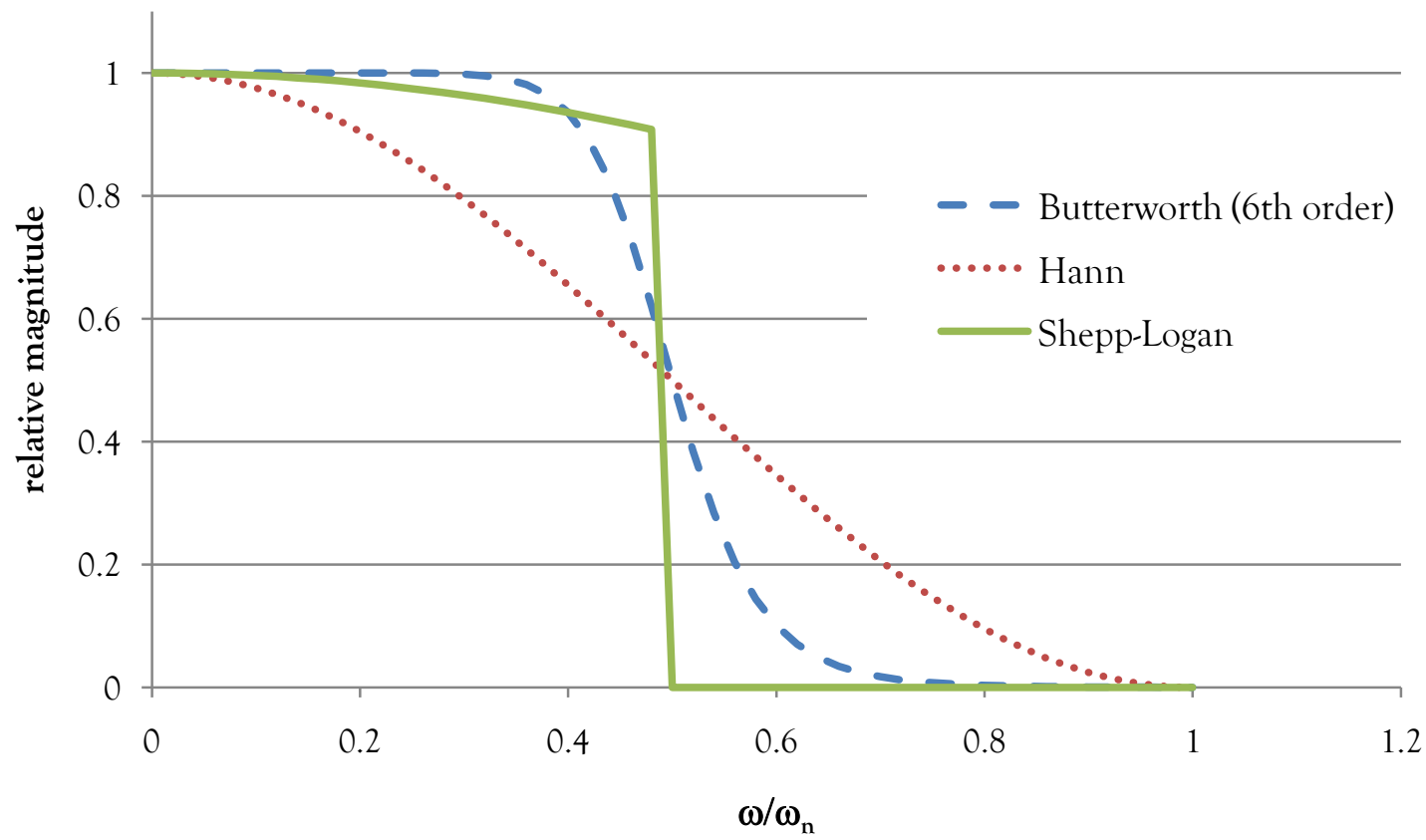

Figure 2-4. Typical windowing functions used to roll off high frequencies for filtered back-projection. (For equivalent $-3 \mathrm{~dB}$ frequencies, the cut-off frequency of the Shepp-Logan filter and the critical frequency of the Butterworth filter are set to 0.5 of the Nyquist frequency $\left(\omega_{n}\right)$ and the cut-off frequency of the Hann window is set to $\omega_{\mathrm{n}}$.)

Filtered backprojection has the advantage of being relatively easy to implement and computationally fast and straight forward, especially since the fast-Fourier transform (FFT) algorithm made the filtering step practical even with the computing power available at the time CT 
and SPECT were being developed. For this reason, filtered backprojection has also been used to reconstruct emission tomography data from PET and SPECT scans.

Filtered back-projection also requires the projection data to meet certain minimum requirements that ensure the projection data contains sufficient information for reconstruction. This condition the "Orlov" condition for parallel beam data - requires that the projections are not truncated, i.e. none of the object to be reconstructed projects beyond the edge of any of the projections, and the angular trajectory of the detector covers a path on a $4 \pi$ sphere of all possible angles such that every great circle on the $4 \pi$ sphere intersects that path. A circular orbit around an axis parallel to the detector is the simplest and most often used trajectory that meets requirements.

\subsection{I.I Fan and cone beam projection and filtered back-projection}

Clinical CT scanners generate an X-ray beam which originates from a single point, producing a fanbeam (Figure 2-5) rather than the parallel beams considered in the previous section. For fan-beams, the projection data can either be resorted into sets of parallel beams and reconstructed with the standard filtered back-projection algorithm, or alternatively reconstructed directly using an algorithm modified for the fan-beam geometry. The latter is slightly more complicated, but still an exact inversion of the fan-beam projection and avoids the resolution loss from the intermediate interpolation from fan- to parallel-beam geometry. 


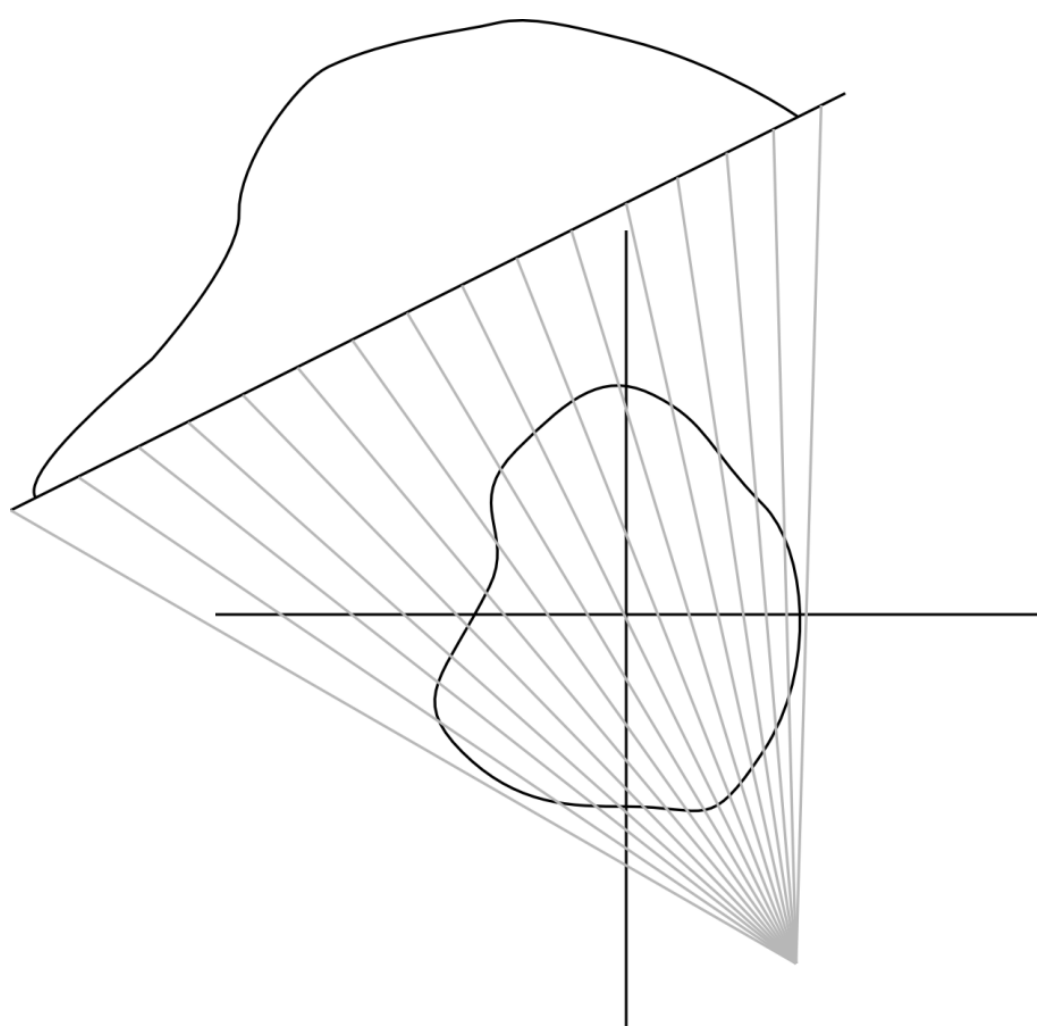

Figure 2-5. Fan-beam projection for transmission imaging.

Cone-beam acquisition extends the fan-beam acquisition along the axial direction, so the lines of summation, other than those in the central plane, are no longer perpendicular to the axis of rotation. An exact analytical inversion of the cone-beam projection was derived by (Tuy, 1983), along with a minimal set of completeness conditions regarding the path of the detector required for accurate reconstructions. Circular cone-beam acquisition doesn't fully meet the Tuy conditions, except for the plane of rotation of the source, but the data can still be reconstructed with reasonable accuracy. The most commonly implemented cone-beam inversion algorithm is the one derived by (FDK) (Feldkamp, Davis, \& Kress, 1984), an algorithm similar to fan-bean reconstruction, but with the projections reweighted according to a heuristically derived formula. Because of the incomplete projection data, FDK backprojection produces images with some blurring in the axial direction that becomes more severe as you move away from the central plane.

The microCT scans for the following work were reconstructed with the FDK algorithm. 


\subsubsection{Filtered backprojection of multiplexed multi-pinhole reconstruction}

Whether or not analytic reconstruction of multiplexed multi-pinhole projection (MMP) data is possible is an open question which, to our knowledge, has not been investigated in any published work to date.

The advantage of developing an analytical reconstruction of MMP projection data would not be for actual reconstruction, but to precisely characterize the limitations of the iterative reconstruction introduced in the following section (\$2.2.2). We know by observation with the NanoSPECT system, for example, that uniform volumes of activity that exceed certain dimensions cannot be correctly reconstructed. For a cylindrical phantom, once the diameter exceeds some threshold, the reconstruction becomes dominated by helical artefacts. In $\$ 3.5$ we describe some rudimentary simulations designed to determine that maximum size of a uniform object that can be accurately reconstructed, but this falls short of a rigorous analytic analysis of the conditions under which some object can be unambiguously reconstructed, analogous to the 'Tuy' or 'Orlov' conditions already mentioned.

\subsubsection{Iterative reconstruction methods}

In contrast to analytic reconstruction methods which calculate the final reconstruction directly from the known projections, iterative methods start by guessing the reconstruction, then repeatedly modifying that guess to conform more closely to the measured projection data. Iterative methods typically require an order of magnitude more computer processing power to converge to an acceptable solution. There is not always a clear answer as to when a solution is "close enough", i.e. how many iterations should be used, and there's no guarantee that the solution found by an iterative algorithm will be unique. 
Numerous algorithms have been proposed for iterative reconstruction. Algebraic reconstruction techniques (ART) (Gordon, Bender, \& Herman, 1970) was one of the first algorithms suggested, followed by simultaneous iterative reconstruction techniques (SIRT) (Gilbert, 1972) with additive (simultaneous algebraic reconstruction technique, SART), and multiplicative variants (simultaneous multiplicative algebraic reconstruction technique, SMART). Each of these techniques follows a systematic process of progressively updating the values in the reconstructed image to arrive at a solution which results in projections similar to the measured projections. These methods have been replaced by Maximum Likelihood Expectation Maximization (ML-EM) and a derivative variant of ML-EM, Ordered Subsets Expectation Maximization (OS-EM), which converges much faster. Unlike the earlier algorithms, ML-EM explicitly considers the noise properties of the acquired projection data and converges towards the solution which maximizes the likelihood of the observed projections assuming Poisson statistics. (If the noise in the projection data is assumed to be Gaussian, least-squares minimization approaches can be used instead (Fessler, 1994).) The noise in an image reconstructed with statistical approaches like ML-EM is also Poissonlike - the variance of a given pixel is proportional to that pixel's intensity (Barrett, Wilson, \& Tsui, 1994) (Wilson, Tsui, \& Barrett, 1994). Therefore regions of the reconstruction with low activity also have very low noise. This differs from analytical reconstruction where the noise is approximately constant over the entire reconstruction. The following sections briefly describe the OS-EM algorithm that was used for SPECT reconstruction in this thesis.

\subsubsection{Imaging as a linear operation}

Analytic reconstruction is also only accurate when the projections are consistent. This is a reasonable assumption for most CT imaging, where the number of photons is sufficiently large that the statistical uncertainties in the observed photon densities will be small. In emission tomography however, the number of photons detected at any given pixel on the detector is frequently small 
enough that statistical uncertainty (Poisson noise) will be the dominant source of noise in the projection data. As previously noted, filtered back-projection emphasizes the high frequencies in the projection data, and statistical noise will result in noticeable streaks and artefacts in the reconstruction. Moreover, attenuation and scatter (\$2.3-2.4) also contribute to further inconsistency in the projection data, since the magnitude of both effects depends on the depth of the source within the attenuating medium which changes with projection angle.

For this reason, most emission tomography reconstruction is now done using iterative methods which are capable of modeling the true geometry of the imaging system and seek the statistically most-probable reconstruction in spite of the noise in the projection data.

In any physical implementation of the imaging process, the projections consist of a sampled version of the 'true' projections, and can therefore be represented by a single column vector, $\mathbf{g}$, with each member of $\mathbf{g}$ corresponding to a single pixel in projection space. Likewise, the source distribution is also represented by a discretized model, a column vector $\mathbf{f}$, with each member of $\mathbf{f}$ corresponding to a single voxel in the volume to be reconstructed. Projection of the source distribution into projection space can be described by the linear operation.

$$
\mathbf{g}=\mathbf{H f}+\mathbf{n}
$$

where $\mathbf{H}$ is the 'projection matrix' which defines the projection of $\mathbf{f} \rightarrow \mathbf{g}$, and $\mathbf{n}$ is the noise in the projection data. Each member of $\mathbf{H}, h_{i j}$ specifies the probability of detection in projection bin $i$ for a photon emitted from voxel $j . \mathbf{H}$ is an $n \times m$ matrix, where $n$ is the total number of projection bins and $m$ is the total number of voxels in the reconstruction. For any practical imaging system, $\mathbf{H}$ tends to be fairly sparse, since, because of collimation, the photons emitted from any particular voxel can only be detected by a small subset of the detector pixels. 


\subsubsection{Matrix inversion, pseudo inversion}

The most direct approach to finding $\mathbf{f}$, given $\mathbf{g}$, is direct matrix inversion:

$$
\mathbf{f}=\mathbf{H}^{-1} \mathbf{g}
$$

but this is impractical for any real imaging system, because of the size of $\mathbf{H}$ and the likelihood that $\mathbf{H}^{\mathbf{- 1}}$ doesn't exist (Budinger \& Gullberg, 1974). It also ignores the noise in the projections.

If the noise in the data is assumed to be Gaussian, that is $\mathbf{n}$ is $N\left(0, \sigma_{i}^{2}\right)$, a least-squares solution that seeks to minimize the objective function:

$$
O=\sum_{i} \frac{\left(g_{i}-\sum_{j} h_{i j} f_{i}\right)^{2}}{\sigma_{i}^{2}}
$$

Can be explicitly found:

$$
\mathbf{f}=\left[\mathbf{H}^{\mathrm{T}} \boldsymbol{\Phi}^{-1} \mathbf{H}\right]^{\mathrm{G}} \mathbf{H}^{\mathrm{T}} \boldsymbol{\Phi}^{-1} \mathbf{g}
$$

where $\mathbf{A}^{\mathrm{G}}$ is the generalized inverse, or pseudo-inverse of $\mathbf{A}$, and $\mathbf{\Phi}=\operatorname{diag}\left(\sigma_{i}\right)$ is the covariance matrix. This deals with the noise, assuming the noise is Gaussian, but still requires manipulation of impractically large matrices.

Given the difficulties of inverting the imaging process directly, almost all image reconstruction uses iterative methods for solving the system of equations to find $\mathbf{f}$.

\subsubsection{Maximum Likelihood - Expectation Maximization (ML-EM)}

Because emission tomography involves discrete processes, namely nuclear decay and photon detection, the actual counts observed in any given pixel cannot be calculated deterministically even if the source distribution were known a priori. Instead of additive noise as above, the projection operation can be written in terms of an expectation value for each projection bin, $g_{i}$ : 


$$
E[\mathbf{g}]=\mathbf{H f}
$$

The most probably source distribution $\mathbf{f}$ is therefore assumed to be that which maximizes the likelihood of observing the projection data $\mathbf{g}$.

$$
\hat{\mathbf{f}}=\arg \max _{\mathbf{f}} p(\mathbf{g} ; \mathbf{f})
$$

Here, $p(\mathbf{g} ; \mathbf{f})$ is the probability of observing the projection data $\mathbf{g}$ if the source distribution is specified by $\mathbf{f}$. For a SPECT imaging system, $\mathbf{g}$ consists of Poisson variables determined by $\mathbf{H f}$; the joint probability of which can be stated as follows:

$$
p(\mathbf{g} ; \mathbf{f})=\prod_{i} \frac{\left(\sum_{j} h_{i j} f_{j}\right)^{g_{i}} \exp \left[-\sum_{j} h_{i j} f_{j}\right]}{g_{i} !}
$$

(Shepp and Vardi 1982), following the generalized expectation-maximization (EM) algorithm described by (Dempster, Laird and Rubin 1977), have shown that the maximum likelihood (ML) solution for $\hat{\mathrm{f}}$, which maximizes the probability of $\mathbf{g}$, can be found iteratively by the following algorithm:

$$
\hat{f}_{j}^{(n+1)}=\hat{f}_{j}^{(n)} \frac{1}{\sum_{i^{\prime}} h_{i^{\prime} j}} \sum_{i} h_{i j} \frac{g_{i}}{\sum_{k} h_{i k} \hat{f}_{k}^{(n)}}
$$

This algorithm is remarkably intuitive. The EM reconstruction algorithm begins with an estimate of the source distribution, which is then 'projected' to obtain the projection set that would be expected if the estimate were the true source distribution. This hypothetical projection set is then compared to the true (observed) projections. The ratios of the 'true' projection data to the hypothetical projection data are then backprojected to determine a set of scaling factors which are then applied to the current estimate of the source distribution to obtain a new estimate for the 
source distribution (Figure 2-6). This algorithm has been shown by Shepp and Vardi to converge monotonically to the maximum likelihood solution assuming Poisson noise in the projection data.

The ML-EM algorithm enforces a positivity constraint on the reconstructed data, provided the initial estimate and the projection data are positive. This condition is essential in the reconstruction of physically plausible source distributions.
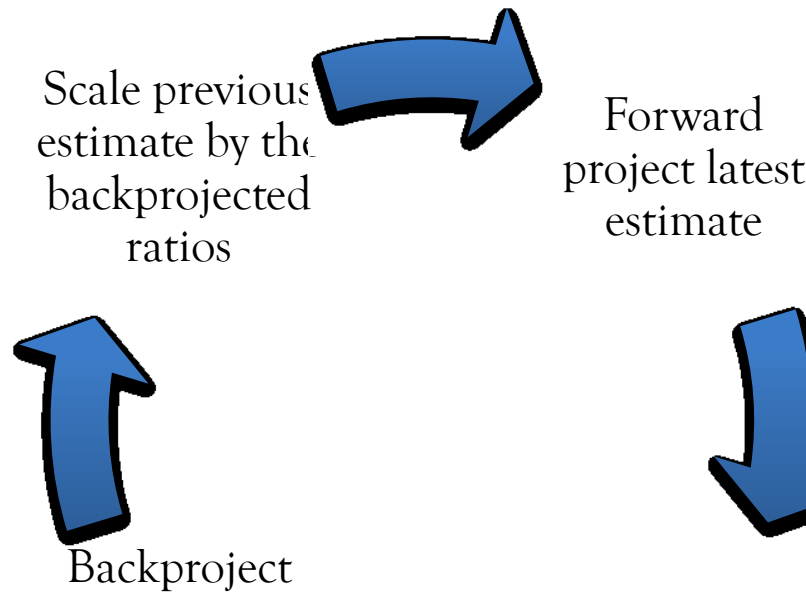

the true-toestimated projection ratio
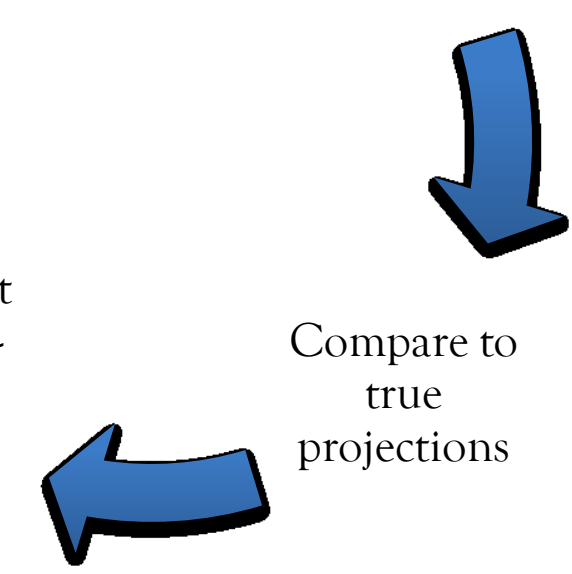

Figure 2-6. Maximum Likelihood-Expectation Maximization (ML-EM) algorithm

\subsubsection{Ordered Subsets - Expectation Maximization (OS-EM)}

ML-EM reconstruction, which requires full forward-and back-projection of the entire data set for each iteration, can be extremely time-consuming, even on a very fast computer. Reconstruction can be sped up by ordered-subsets expectation maximization (OS-EM). In OS-EM, the projection data are divided into multiple subsets and only one of these subsets is used for each successive update of the estimated source distribution (Hudson \& Larkin, 1994) (Byrne, 1996). As defined by Hudson 
and Larkin, each full iteration corresponds to a complete cycle through each of the projection subsets. The OS-EM approach has been shown to speed up convergence by a factor equal to the number of subsets used. Provided the subsets are consistent and balanced (that is, each voxel in the reconstruction contributes approximately equally to all subsets), the OS-EM algorithm can be shown to approximately converge to the maximum-likelihood solution for the source distribution. True convergence is only possible for completely consistent (i.e. noise-free) projection data. Inconsistent data will converge to a set of solutions which cycle at each sub-iteration. In practice, for realistic projection data, provided the number of subsets isn't too large, the difference between the ML-EM and OS-EM solutions is small (Hudson \& Larkin, 1994).

\subsection{Attenuation correction}

Between the point of origin and the detector, the SPECT photons pass through varying amounts of attenuating media before reaching the camera. For photons in the energy range of most SPECT isotopes $(>70 \mathrm{keV})$ passing through typical biological materials (water, fat, muscle, bone), the most likely interaction is Compton scatter, where the photon loses some energy and scatters. For relatively low energy photons, as in ${ }^{125} \mathrm{I}$ imaging $(27-35 \mathrm{keV})$ photoelectric absorption may also contribute significantly to total photon attenuation. Both the photoelectric effect and Compton scatter lead to a loss of primary photons, and therefore contribute to attenuation. In addition, those photons which are Compton scattered may still be detected and be difficult to distinguish from primary photons (see §2.4).

In transmission imaging, the intensity at each point on the detector, $p_{\phi}(\mathbf{r})$, is as follows:

$$
p_{\phi}(\mathbf{r})=\exp \left\{-\int_{L} \mu(\mathbf{x}(\ell)) d \ell\right\}
$$


where $L$ is the path from the radiation (x-ray) source to point $\mathbf{r}$ on the detector. In this case linear attenuation coefficient is the property of interest, and the goal of reconstruction is to recover a tomographic map of the attenuation coefficients, as discussed in the previous section. (The picture is complicated slightly because the $\mathrm{x}$-ray beam is almost always poly-energetic, and $\mu$ changes as the lower energy photon are absorbed, even in a homogenous medium. This phenomenon, beam hardening, is discussed and characterised for the NanoSPECT/CT system in §3.2.1.)

For emission imaging,

$$
p_{\phi}(\mathbf{r})=\int_{V(\mathbf{r})} f(\mathbf{x}) \exp \left\{-\int_{L(\mathbf{x} \rightarrow \mathbf{r})} \mu\left(\boldsymbol{x}^{\prime}(\ell)\right) d \ell\right\} d \mathbf{x}
$$

Where $V$ is volume of the source distribution 'visible' to point $r$ on the detector, $L(\mathbf{x} \rightarrow \mathbf{r})$ is the path from $\mathbf{x}$ to $\mathbf{r}$, and $\mu(\mathbf{x})$, is the linear attenuation coefficient of the SPECT photons at $\mathbf{x}$. Here, $f(\mathbf{x})$ is the quantity of interest, and the attenuation, $\mu(\mathbf{x})$, serves only to complicate its accurate recovery.

\subsection{Attenuation correction in clinical imaging}

As a consequence of attenuation, photons from deeper within a subject are less likely to reach the detector, resulting in depressed activity on the reconstructed image deeper within the subject. Attenuation also reduces the overall efficiency of the imaging system and makes quantitative SPECT measurements impossible without first correcting for the lost photons. The total amount of attenuation depends on the subject habitus, projection angle, and the isotope being used.

The challenges to quantitative accuracy created by photon attenuation have been recognized since the origins of SPECT imaging. Several methods for attenuation correction were suggested by (Budinger \& Gullberg, 1974). One approach was to assume a constant linear attenuation coefficient, and calculate the attenuation based on the path length between each voxel and the edge 
of the object being imaged. Budinger \& Gullberg apply this method in conjunction with a leastsquares iterative reconstruction algorithm. They also suggest several alternatives for estimating attenuation that they do not investigate further:

i. correcting the projection data by rescaling along each projection ray based on the geometric mean of the conjugate projections and a factor that depends on the thickness of the body, the linear attenuation coefficient, and the distribution of the isotope,

ii. calculating both tracer concentration and attenuation using an iterative reconstruction algorithm (ART) that alternates between updating the estimate of the former and the later,

iii. measuring attenuation using transmission methods and a ${ }^{99 \mathrm{~m}} \mathrm{Tc}$ or ${ }^{57}$ Co source, or

iv. using multiple isotopes with different attenuation coefficients. In this case, the known attenuation coefficients for each of three isotopes for lung, soft-tissue, and bone, leads to a system of equations that can be used to calculate the total attenuation for each line of response. (This approach seems more theoretically novel than practically useful.)

\subsection{I.I Chang correction}

The most frequently implemented approach to SPECT attenuation correction is first described in (Chang, 1978). Chang proposed a post-reconstruction correction method whereby after reconstructing the SPECT image by filtered back-projections, each voxel of the reconstruction is scaled by a correction factor:

$$
c(\mathbf{x})=\frac{1}{\frac{1}{M} \sum_{i=1}^{M} \exp \left(-\mu \ell_{\theta_{i}}\right)}
$$

Here, $\mu$ is the attenuation coefficient of the subject, $\ell_{\theta_{i}}$ is the distance from the voxel at $\mathbf{x}$ to the perimeter of the subject in the direction of the ith projection, and $\mathrm{M}$ is the total number of projections (Figure 2-7). This approach requires knowledge of only the boundary of the subject being imaged and an average attenuation coefficient inside the subject. Chang correction is exact for reconstruction of a point source in a uniformly attenuating medium, but not for extended source distributions. The quantitative accuracy can be improved by an iterative approach. After 
correcting the initial reconstruction, the corrected results are forward-projected and subtracted from the original projections. The error projections are then reconstructed, attenuation correction corrected, and added to the initial (corrected) reconstruction.

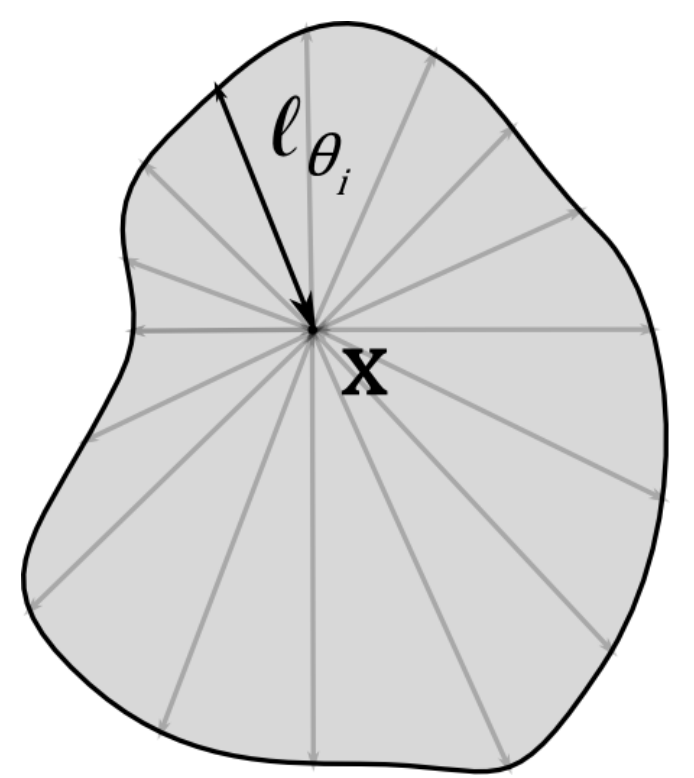

Figure 2-7. The Change attenuation correction method applies a correction factor for each point (x) in the reconstructed image by calculating the attenuation between that point and the border of the object for each projection angle and finding the mean. The reconstructed image is then scaled by the inverse of the mean attenuation. The original method uses the lengths shown and uniform attenuation, later work uses non-uniform attenuation maps.

Although this method is only analytically exact in the case of a point source and not for an extended distribution - it has the advantage of being fast to implement (essential given the available computing hardware of the 1970s and 1980s), and reasonably accurate. Chang correction can be further improved by using non-uniform attenuation maps (Manglos, Jaszczak, \& Floyd, 1988).

\subsubsection{Analytic correction}

Various exact inversions of the attenuated acquisition problem have also been described for the case of uniform attenuation where at least the contour of the subject body is known (Bellini, Piacentini, Cafforio, \& Rocca, 1979) (Tretiak \& Metz, 1980) (Tanaka, Toyama, \& Murayama, 1984). Exact analytic inversions of the attenuated Radon transform for non-uniform attenuation have also been published. See, for example (Natterer, 2001). 
Although analytic reconstruction methods are exact in the absence of noise, the projection data in most nuclear medicine scans contains substantial Poisson noise because of the discrete nature of the detection and the relatively low numbers of photons detected at any given location on the detector. As already noted, the presence of noise in the projections significantly degrades the quality of images reconstructed by filtered back-projection; the exact inversion formulae tend to further amplify the statistical noise.

\subsection{I.3 Iterative reconstruction with attenuation correction}

With iterative reconstruction attenuation can be modeled and therefore corrected as part of the projection and back-projections operations (Gullberg, Heusman, Malko, Pelc, \& Budinger, 1986) (Tsui, et al., 1989) (Blankespoor, et al., 1996). To correct for attenuation, a map of the linear attenuation coefficients for the isotope being used is an input to the SPECT reconstruction algorithm. These maps can be measured by radioisotope transmission scans or derived from a CT scan of the subject (Willowson 2008).

Implementation of this approach in the context of multiplexed, multi-pinhole, small-animal SPECT was one of the major goals of the research in this thesis. Attenuation in pre-clinical imaging of small-animals is often ignored, since the magnitude of attenuation is much less than that in humans. However, the attenuation is not negligible; Monte Carlo modelling of a single-pinhole SPECT scan of a ${ }^{99 \mathrm{~m}} \mathrm{Tc}$ source at the centre of a rat-sized water cylinder (50 mm diameter), demonstrates that attenuation degrades the quantitative accuracy by $25-30 \%$ below the true value (Hwang, Franc, Gullberg, \& Hasegawa, 2008). For isotopes which emit lower energy radiation such as ${ }^{201} \mathrm{Tl}$ or ${ }^{125} \mathrm{I}$, the effect of attenuation will be even more significant. 
For a mouse size phantom, the magnitude of the attenuation is less, but still significant. According to Monte Carlo simulations by (Chen, Wang, Lee, \& Tsui, 2009), attenuation leads to an $18 \%$ reduction in measured activity in with ${ }^{99 \mathrm{~m}} \mathrm{Tc}$ or ${ }^{111} \mathrm{In}$, and a $41 \%$ reduction using ${ }^{125} \mathrm{I}$.

\subsection{Scatter}

Compton scattering changes a photon's direction such that the positional information about its origin is lost, and also changes its energy. Ideally, scattered photons could be identified by their lower energy and ignored by the camera. However, the sodium iodide Anger camera used in most clinical SPECT scanners, and in the nanoSPECT system of interest to this work, has an energy resolution of about $10 \%$ at ${ }^{99 \mathrm{~m}} \mathrm{Tc}$ energy $(140 \mathrm{keV})$ (Bioscan, 2007). For SPECT acquisition, an energy window of $140 \mathrm{keV} \pm 10 \%$ is used, which allows photons with energies $126-154 \mathrm{keV}$ to be accepted as primary photons. In terms of the energy of the scattered photons, by rearranging the Compton equation:

$$
\lambda^{\prime}-\lambda=\frac{h c}{E^{\prime}}-\frac{h c}{E}=\frac{h}{m_{e} c}(1-\cos \theta)
$$

where $\mathrm{E}$ and $\mathrm{E}$ ' are the photon energies before and after scattering, it can be shown that photons scattered by as much as $53^{\circ}$ will still have enough energy to be detected within the photopeak window. Multiply scattered photons may also contribute to the photons detected within the photopeak energy window.

\subsection{Scatter estimation and compensation}

Several methods have been proposed to estimate and compensate for scatter, including the undercorrection for attenuation (broad-beam scatter correction), the Compton-window, or dual energy window method (DEW), the triple energy window (TEW) method, deconvolution methods, and model based approaches. A thorough review of scatter compensation methods for emission tomography can be found in (Ziadi \& Koral, 2004). Here we briefly review a few of the more 
common methods, with focus on broad-beam attenuation and dual energy window scatter correction, which we implement in small animal imaging. Although these two methods are not the most accurate, scatter is already expected to be a relative small contributor to quantitative error in small animal imaging, and more complex approaches such as those mentioned in \$2.4.1.4 are not expected to yield results that would justify the added computational demands.

\subsection{I.I Broad-beam attenuation scatter correction}

Since, for an extended distribution, scatter is likely to result in more photons scattered into the detector, leading to an overestimation of activity, while attenuation reduces the number of detected photons, one way of compensating for scatter is to under-correct for attenuation (Larsson \& Israelsson, 1982). This is sometimes referred to as broad-beam scatter correction, since it uses the attenuation measured for a broad-photon beam, where photons scatter into, as well as out of, the detected beam. This approach is simple, but the success may vary for non-trivial arrangements of the source and the attenuating/scatting media.

\subsection{I.2 Deconvolution methods}

The primary effect of scatter on the projection data is a broadening of the point spread function (PSF). Thus one way to correct for scatter is by deconvolution of the projection image to undo the low-pass filtering introduced by scatter (Yanch, Flower, \& Webb, 1990). Unfortunately, this method also tends to amplify the high-frequency noise present in the projection data. It also does not account for heterogeneity in scatter contribution due to differences in depth of photon emission and the heterogeneity of the scattering media. A more sophisticated method of deconvolution, transmission-dependent convolution subtraction (Meikle, Hutton, \& Bailey, 1994), takes into account the depth-dependant nature of the scatter contribution, and attempts to iteratively estimate and remove the scatter using conjugate projections and a transmission scan of the subject. 


\subsection{I.3 Dual energy window scatter correction}

The dual-energy window (DEW) or Compton-window method estimates the scatter in the projections by also recording photons detected in an energy window below the photopeak window (Figure 2-8). From the number of presumably scattered photons in the lower energy window, the number of scattered photons in the photopeak window can be estimated. This approach to scatter subtraction was originally employed for planar imaging by (Bloch \& Sanders, 1973), and applied to SPECT imaging by (Jaszczak, Greer, Floyd, Harris, \& Coleman, 1984).

The DEW method depends on a parameter, $\mathrm{k}$, which is the ratio of scattered photons in the photopeak window to photons in the lower energy window. Unfortunately, this scatter multiplier is not a fixed value and but depends on several factors, including the distribution and nature of the scattering media, the nature of the imaging task (cold lesion in hot background or hot lesion in a cold background), the method of reconstruction (filtered backprojection or iterative) and the size of the ROI used to evaluate quantitative accuracy. In their initial work, Jaszczak et al. imaged a line source in air and in water, reconstructed the photopeak and scatter projections. The $\mathrm{k}$ value was determined to be 0.5 heuristically based on the fraction of the scatter reconstruction which, when subtracted from the reconstruction of the line source in water, resulted in the same radial profile as for the line source imaged in air. Later work by (Koral, Swailem, Buchbinder, Clinthorne, Rogers, \& Tsui, 1990) involving imaging spheres of activity in a uniform background showed that $\mathrm{k}$ was not sensitive to sphere location or activity ration (lesion-to-background). But, the $\mathrm{k}$ value did depend slightly on the size of the scattering media, and on the reconstruction procedure - whether the scatter was subtracted from the projections before reconstruction or if the photopeak and scatter projections were reconstructed separately and then subtracted. However, since this work was performed with clinical scanners and these results may not translate directly to the significantly 
different geometry of pre-clinical imaging systems. In this work we investigate the application of DEW scatter correction to small animal SPECT.

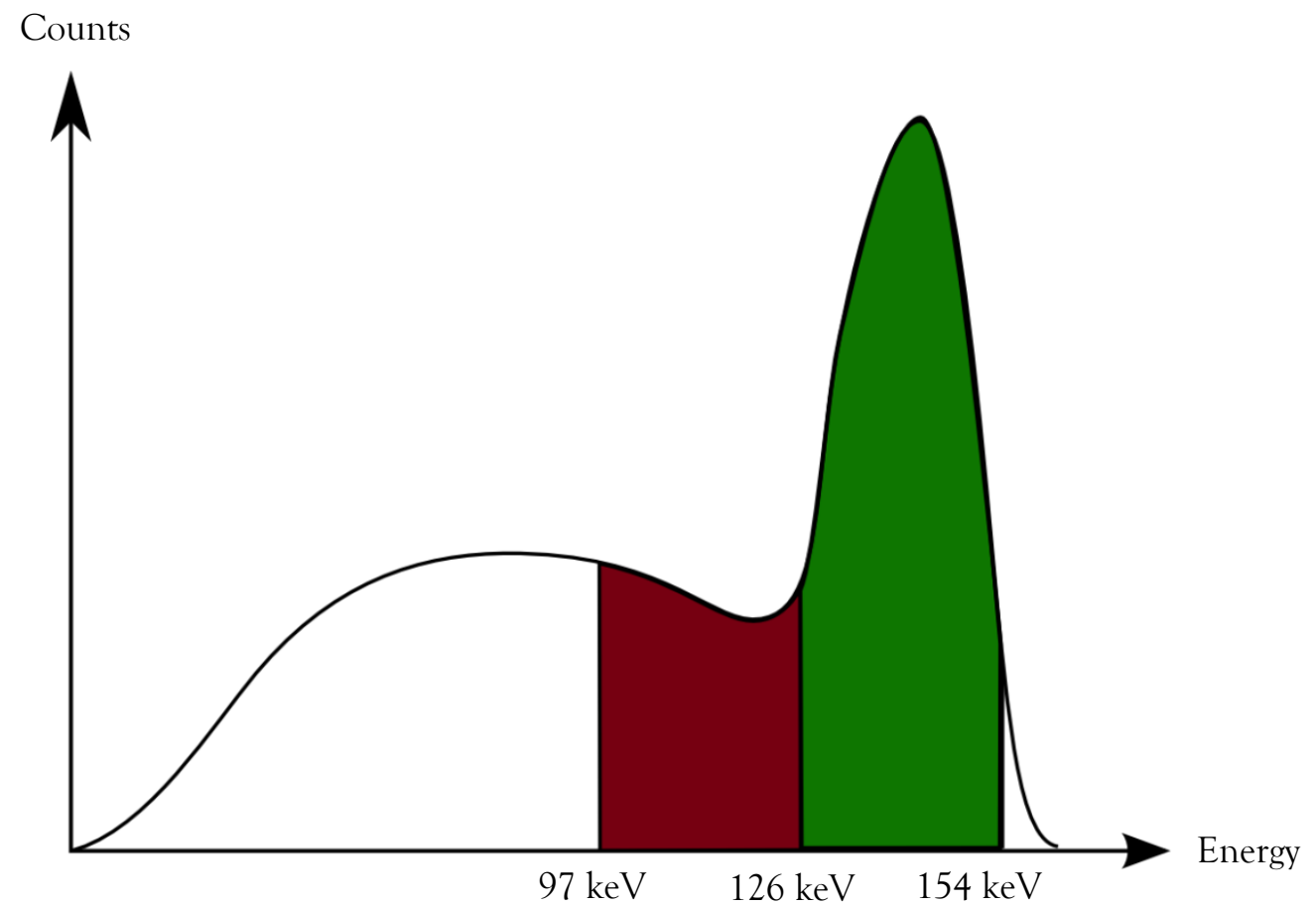

Figure 2-8. Energy windows used for dual energy window scatter correction of ${ }^{99 \mathrm{~m}} \mathrm{Tc}$ images. The green photopeak window contains the unscattered primary photons plus some scattered photons. The number of photons detected in the red downscatter window is used to estimate the number of scattered photons in the photopeak window.

\subsubsection{Other scatter correction methods}

The triple energy window (TEW) method, like the DEW method, estimates scatter based on the number of photons detected in energy windows adjacent to the photopeak window (Ogawa, Harata, Ichihara, Kubo, \& Hashimoto, 1991). For TEW, two energy windows are used to estimate scatter - one above and one below the photopeak window. The number of scattered photons in the photopeak window is estimated to be the mean number of the photons detected by the two windows, scaled for the width of the scatter windows and the width of the photopeak window. Unlike the DEW method, TEW scatter estimation does not depend on estimating a $\mathrm{k}$ parameter, and can also be used to estimate the number of down-scattered photons from higher energy isotopes in multi-isotope imaging. Where DEW scatter correction uses a down-scatter window of 
similar or equal width to the photopeak window, the energy windows used by TEW are narrower and immediately adjacent to the upper and lower bounds of the photopeak window such that the spatial distribution of photons in the scatter windows more accurately represents that in the photopeak window.

The distribution of Compton scattering angles is well characterized by the Klein-Nishina equation and therefore it is possible to calculate analytically the expected scatter distribution for a particular distribution of source activity and scattering media (Wells, Celler, \& Harrop, 1998). Alternatively, one can model the scatter distribution using combinations of pre-determined point-spread functions or Monte Carlo techniques (Frey \& Tsui, 1993) (de Jong \& Beekman, 2001) (Beekman, de Jong, \& van Geloven, 2002). These methods are much more computationally demanding than the energy-window or convolution-based methods.

\subsection{Quantitative small animal SPECT}

The earliest work on quantitative small animal pinhole SPECT is a paper by ( $\mathrm{Li}$, Jaszczak, \& Coleman, 1995). Using a filtered backprojection reconstruction algorithm with Chang attenuation correction, DEW scatter correction, and images acquired with a pinhole aperture mounted on a clinical camera, they were able to recover the activities of point sources within a cylindrical water phantom and the activity concentration within a uniform cylindrical phantom with less than $7 \%$ error.

Semi-quantitative measurements of dopamine transporters in the mouse brains were performed by (Acton, Choi, Plössl, \& Kung, 2002). They reported excellent correlation between distribution volume ratios (DVR) measured using a clinical SPECT camera with custom pinhole collimators, and the same DVRs measured ex vivo. They also reported test-retest repeatability of DVR measurements made with SPECT to be better than 3\%. 
The earliest experimental work using a dedicated small animal scanner for quantitative SPECT is (Hwang \& Hasegawa, 2005), who demonstrate improved quantification of a $38 \mathrm{~mm}$ diameter uniform cylindrical phantom containing ${ }^{125}$ I. The SPECT and CT scans were acquired with an XSPECT/CT scanner (Gamma Medica, Inc.). Including CT-based attenuation correction eliminated a $30 \%$ depression in activity at the center of the phantom relative to the edges and reduced the standard deviation of the pixel values from $10 \%$ to $6 \%$. All SPECT analysis by Hwang and Hasegawa was done in relative terms, and no attempt was made to recover the quantitative activity concentration.

This work was extended to acquisition of rat images in (Hwang, Taylor, VanBrocklin, Dae, \& Hasegawa, 2006), where the same group demonstrated that the circumferential activity profile of a rat myocardium became more uniform with the inclusion of attenuation correction in the reconstruction. Additionally, they also showed that attenuation correction did not increase the noise of the reconstructed image. As in the previous study, images were acquired using ${ }^{125} \mathrm{I}$ as the isotope and the X-SPECT/CT small animal imaging system.

In vivo quantification for a Linoview SPECT system was investigated by (Walrand, Jamar, de Jong, \& Pauwels, 2005). They injected two mice with two different tracers - one with ${ }^{99 \mathrm{~m}} \mathrm{Tc}$, the other with ${ }^{111} \mathrm{In}$, both designed to be taken up in the kidneys - and measured activity concentrations using SPECT that agreed with those measured by direct measurement of excised kidneys in a gamma counter. Attenuation correction was not applied as part of the reconstruction, but the activity measured using SPECT was corrected based on the assumption of $1 \mathrm{~cm}$ soft tissue equivalent attenuation.

Absolute in vivo quantification of kidney uptake of a radionuclide in rats was reported by (Forrer, et al., 2006). This study, performed with the nanoSPECT/CT scanner, used a rat-sized phantom 
containing a known amount of activity to calibrate the scanner, allowing voxel values to be directly converted to activity levels. Activity levels measured with SPECT were compared with those measured directly in a well counter and demonstrated to be well correlated $\left(\mathrm{r}^{2}=0.95\right)$, over a wide activity range.

A thorough discussion of the magnitude of the errors introduced by attenuation, scatter, and partial volume effects can be found in (Hwang, Franc, Gullberg, \& Hasegawa, 2008). Using Monte Carlo they estimate attenuation losses for single pinhole SPECT of a rat-sized water cylinder to be approximately $25 \%$ for ${ }^{99 \mathrm{~m}} \mathrm{Tc}$ and $50 \%$ for ${ }^{125} \mathrm{I}$. For the same phantom, they also estimate scatter-toprimary ratios to be $10 \%$ and $30 \%$ for ${ }^{99 \mathrm{~m}} \mathrm{Tc}$ and ${ }^{125} \mathrm{I}$ respectively. Still using simulated data, they calculated a $10 \%$ overestimate of radioactivity concentration in ${ }^{125} \mathrm{I}$ images reconstructed with only attenuation correction. Interestingly, they observe no overestimation in the ${ }^{99 \mathrm{~m}} \mathrm{Tc}$ simulations reconstructed with the same parameters. Attenuation was evaluated experimentally by imaging a $250 \mu \mathrm{L}$ microsphere containing ${ }^{99 \mathrm{~m}} \mathrm{Tc}$ at the centre of a $32 \mathrm{~mm}$ cylinder. With CT based attenuation correction as per (Hwang \& Hasegawa, 2005), they successfully acquired consistent reconstructed activity with and without water around the source.

Similar Monte Carlo simulations by (Chen, Wang, Lee, \& Tsui, 2009), also based on the geometry of the X-SPECT/CT system, reported underestimation of activity in mouse sized phantoms of $-18 \%$ for ${ }^{99 \mathrm{~m}} \mathrm{Tc}$ and ${ }^{111} \mathrm{In}$, and $-41 \%$ for ${ }^{125} \mathrm{I}$ due to attenuation effects alone. Including scatter in the simulations partially compensated for the attenuation losses, resulting in quantitative degradation of $-12 \%\left({ }^{99 m} \mathrm{Tc},{ }^{111} \mathrm{In}\right)$ and $-21 \%\left({ }^{125} \mathrm{I}\right)$. For rats, they estimated the combined effects of attenuation and scatter to lead to $-21 \%\left({ }^{99 \mathrm{~m}} \mathrm{Tc},{ }^{111} \mathrm{In}\right)$ and $-37 \%\left({ }^{125} \mathrm{I}\right)$ errors in quantitative activity measurement. 
The first study to apply both attenuation and scatter correction to in vivo small animal SPECT is (Vanhove, Defrise, Bossuyt, \& Lahoutte, 2009). This study acquired phantom and animal SPECT scans with a clinical camera fitted with single- and three-pinhole apertures, and CT scans with a micro-CT system. Images were reconstructed with uniform and non-uniform (CT-based) attenuation correction and DEW and TEW scatter correction. For the phantom studies, nonuniform attenuation correction with either TEW or DEW scatter correction accurately recovered the activity concentrations, with uncertainties of approximately $6-8 \%$. In the animal study, the most accurate reconstructions were those which modelled attenuation only. The uncertainties in the animal study were on the order of $10-13 \%$.

Post-reconstruction "Chang" correction was applied to small animal SPECT data acquired with the U-SPECT-II multi-pinhole system by (Wu, van der Have, Vastenhouw, Dierckx, Paans, \& Beekman, 2010). The animal outline used to calculate the Chang correction was acquired with three optical cameras. Scatter correction was applied using the TEW method to estimate scatter and adding the scatter estimate in the forward projection step of the reconstruction. In phantom studies, attenuation and scatter correction reduced the quantification error from $-18.7 \%$ with no correction to $-1.7 \%$. In vivo, quantitative errors with no correction were between $-23.6 \%$ and $-9.3 \%$. After correction, quantitative errors were reduced to between $-6.3 \%$ and $+4.3 \%$.

Finally, (Vanhove, Defrise, Bossuyt, \& Lahoutte, 2011), in addition to attenuation and scatter correction, also incorporated CT-based boundary information into the iterative reconstruction algorithm to encourage smoothness in the SPECT data over uniform regions in the CT data. Though they demonstrate improvements - less noise for equivalent quantitative accuracy - for the reconstruction of phantom data, they acknowledge the limitations of applying the method to small 
animal images where the CT contrast in soft tissue is too small to delineate clear boundaries between organs.

To date, all but one of the published studies on in vivo quantification dealt with either single pinhole SPECT, or multi-pinhole SPECT without multiplexing. Furthermore, much of the published work reports on simulation studies, with only a small number of physical scans acquired for validation. The in vivo study that did use the nanoSPECT camera to quantify the activity in rat kidneys, only used two rats and applied heuristic attenuation correction estimated for the kidneys, not full attenuation modelling. One of the goals of this work is to calibrate the nanoSPECT system to acquire absolute quantitative images with corrections for attenuation and scatter that are robust across a range of subject sizes. 


\section{Acquisition and Reconstruction of SPECT/CT Images}

\section{I NanoSPECT hardware}

The theoretical background of the development of the multiplexed multi-pinhole small animal SPECT scanner which eventually developed into the nanoSPECT scanner is detailed in (Schramm, Ebel, Engeland, Schurrat, Béhé, \& Behr, 2003).

\section{I.I SPECT scanner}

The SPECT portion of the nanoSPECT/CT system comprises four flat panel $\mathrm{NaI}(\mathrm{Tl})$ detectors arranged approximately $175 \mathrm{~mm}$ from the central axis of the scanner. A shielding pyramidal collimator over each detector prevents scattered photons from one detector reaching an adjacent detector. Interchangeable pinhole apertures are mounted on the collimator. The four detector assemblies are mounted on a rotating gantry.

A heated animal bed (Minerve, Esternay, France) with support for inhalation anaesthesia is mounted on a translating stage that moves the subject horizontally along the axis of the scanner. 
Acquisition of SPECT and CT scans with the nanoSPECT/CT system is done with a step-andshoot' procedure. By moving the subject horizontally between each projection or not either helical or circular scans can be obtained.

\section{I.I.I Apertures}

The available apertures for the nanoSPECT system vary in the size and arrangement of the pinholes, and are available in rat and mouse varieties. For rat imaging, the aperture sits on the collimators such that the distance from the central axis to the centre of the central aperture is 45 $\mathrm{mm}$, and the focal length of the pinhole collimator (distance from aperture to detector) is approximately $131 \mathrm{~mm}$. Mouse apertures are set closer to the axis of rotation, resulting in a longer focal length and greater magnification, which allows for higher resolution imaging over a smaller field of view. Both rat and mouse apertures are available in high-efficiency, standard, and highresolution variants, with progressively smaller pinhole diameters, allowing for better resolution, but at the cost of reduced efficiency.

The apertures consist of a flat tungsten slab with one or more pinholes. In our study we used the high resolution rat (APT2) aperture plate with 9 pinholes per plate arranged in 3 rows of 3 pinholes. The relevant dimensions of the APT2 collimator are shown in Figure 3-1. The pinholes of the APT2 aperture plate are angled such that even though the rows of pinholes are offset, the projections fall in a rectangular $3 \times 3$ pattern on the detector surface (Figure 3-2). Overlapping the projections on the detector permits greater magnification and more efficient use of the available detector area, but with some trade-offs in the ability to accurately reconstruct extended objects. 


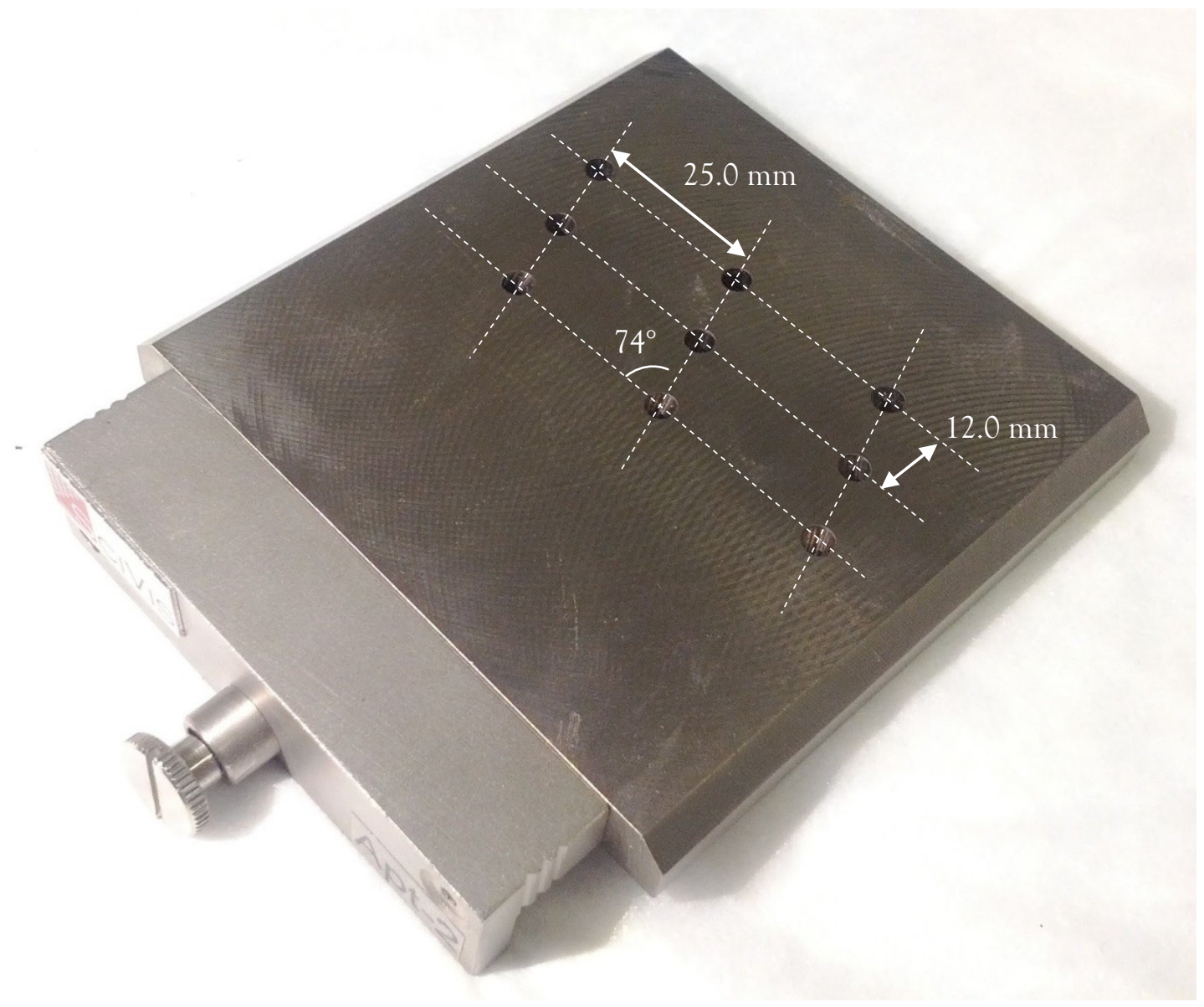

Figure 3-1. Dimensions of the APT2 aperture plate used for rat imaging.

According to the manufacturer, the APT2 apertures, with pinhole diameters of $2.5 \mathrm{~mm}$, permit an optimal image resolution of $1.9 \mathrm{~mm}$, and a maximum efficiency of $2200 \mathrm{cps} / \mathrm{MBq}(0.22 \%)$ of injected activity (Bioscan, 2007).

Resolution can be difficult to specify precisely in the case of pinhole SPECT since it depends on the distance between the source and the pinhole, which, for a fixed radius of rotation, changes with each projection, so the achievable resolution is not uniform across the field of view. The poorest resolution is seen along the central axis of the scanner, since points further from the axis will benefit from the magnification in some of the projections. 


\section{I.I.2 Multiplexing of projections}

The arrangement of the pinholes of the APT2 aperture plate results in substantial overlap on the detectors (Figure 3-2). In the extreme case of a source distribution which fills the field of view, about $41 \%$ of the detector space will contain information from only a single pinhole. Thirty-six percent of the detector pixels will contain information from 2 pinholes, $12 \%$ from 3 pinholes, and $11 \%$ from four pinholes. At no point on the detector do more than four projections overlap. In practice, the degree of multiplexing depends on the size and geometry of the source distribution. For very small sources, there may be no overlap.

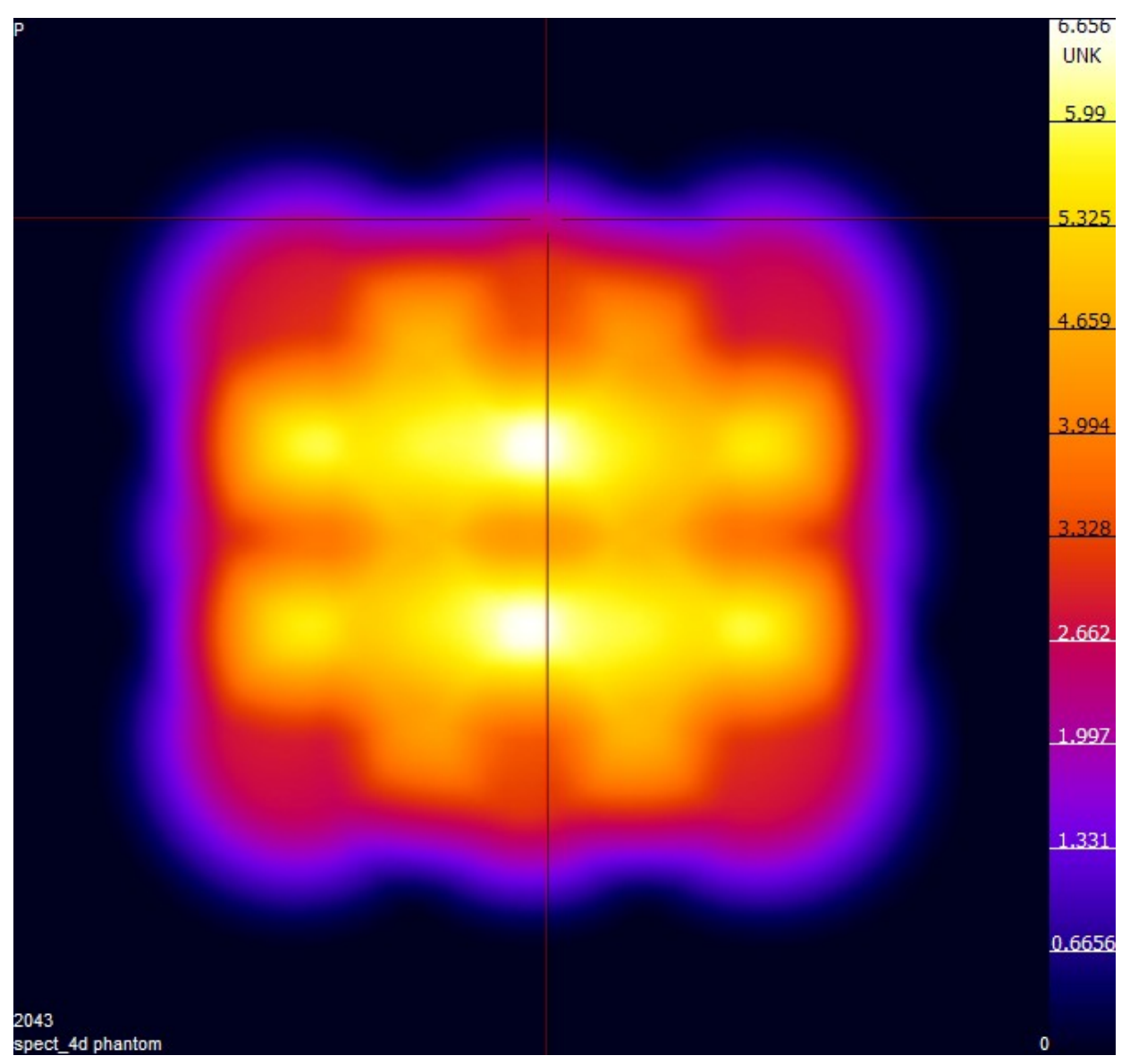

Figure 3-2. Simulated projection of a large cylinder (approx. $50 \mathrm{~mm}$ diameter) that fills the field of view of the APT2 apertures. The result is a $3 \times 3$ array of overlapping projections. Scaling shows relative intensity. 


\section{I.I.3 Detectors}

The detectors use thallium-doped sodium iodide $(\mathrm{NaI}(\mathrm{Tl}))$ crystals which each have an area of $230 \mathrm{~mm} \times 215 \mathrm{~mm}$ and a thickness of $9 \mathrm{~mm}$. The detectors have an intrinsic spatial resolution of approximately $2.1 \mathrm{~mm}$, and an energy range of $25-250 \mathrm{keV}$. The energy resolution at $140 \mathrm{keV}$ $\left({ }^{99 \mathrm{~m}} \mathrm{Tc}\right)$ is $\pm 9.7 \%$.

\subsubsection{CT scanner}

CT scans co-registered to the nuclear medicine scans can be acquired with the cone-beam CT scanner integrated into the NanoSPECT/CT system. X-rays, with a selectable beam energy of 45, 55 , or $65 \mathrm{kVp}$, are emitted from a x-ray tube. The flat-panel detector has dimensions of $49 \mathrm{~mm}$ (axial) $\times 98 \mathrm{~mm}$ (trans-axial). The entire assembly rotates $360^{\circ}$ around the animal bed, with a projection acquired at each $1^{\circ}, 2^{\circ}$ or $4^{\circ}$ interval. Depending on the axial length of the scan, the bed may remain stationary for a circular scan, or move during the scan for helical cone-beam acquisition. The CT scanner transverse FOV is approximately $70 \mathrm{~mm}$, which exceeds the diameter of the animal bed.

\subsection{CT reconstruction software}

The manufacturer supplied CT reconstruction does not correct for x-ray beam hardening, making it difficult to convert the CT image into an attenuation map for attenuation correction. To correct for this, CT reconstruction code was developed for this work which implements the Feldkamp (FDK) cone-beam reconstruction algorithm.

\subsection{Beam hardening correction}

The x-ray beam used for imaging contains a broad spectrum of photon energies. Beam-hardening is the preferential absorption of the lower energy photons and results in a decrease of the effective linear attenuation coefficient of the beam as it passes through an object (Brooks \& di Chiro, 1976). In the reconstructed image, beam hardening results in a 'cupping' artefact, seen in Figure 3-3. 

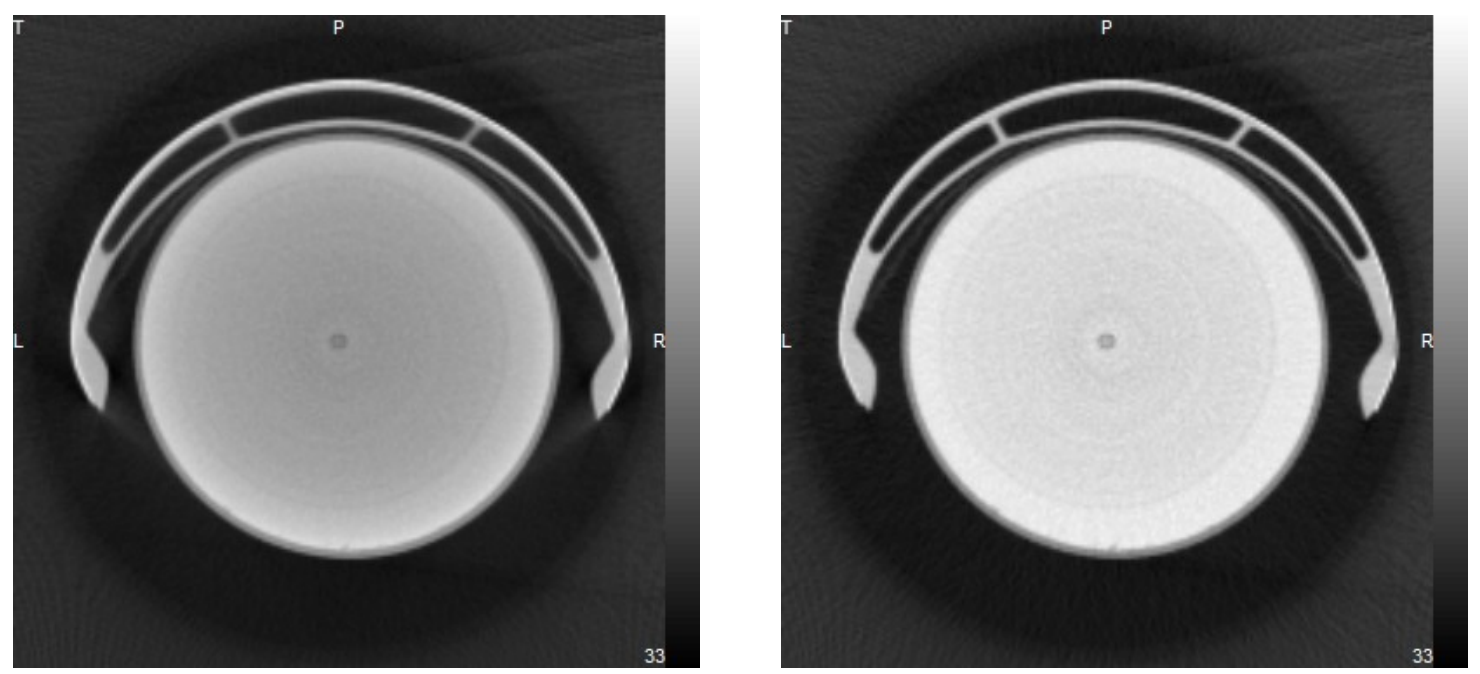

Figure 3-3. CT of a cylindrical water phantom, reconstructed without (left) and with (right) beam-hardening correction. The scanner imaging bed is seen at the top of the image.

For providing anatomical data for co-registration of the nuclear medicine data, the beam hardening artefact poses no problems. However, since we use the CT data to estimate attenuation of the SPECT photons, and model that attenuation in the SPECT reconstruction algorithm (§3.3.1), we corrected for beam hardening by adjusting the CT projection data before reconstructing the CT images. To derive the analytical correction, we filled a container on the animal bed with water, increasing the nominal depth in $5 \mathrm{~mm}$ increments. At each step we acquired a full CT scan of the container. The true water depth was measured from the reconstructed CT image. The depthdependant projection intensity $I(\ell)$, was measured from the mean value within a narrow strip of the projection acquired with the x-ray source directly above the phantom. The intensity with no attenuating media present, $I_{0}$, was measured as the average value in a corresponding region of the blank scan. Attenuation as a function of water thickness, $\ell$ :

$$
p(\ell)=\ln \left(\frac{I_{0}}{I(\ell)}\right)
$$


is plotted in Figure 3-4.

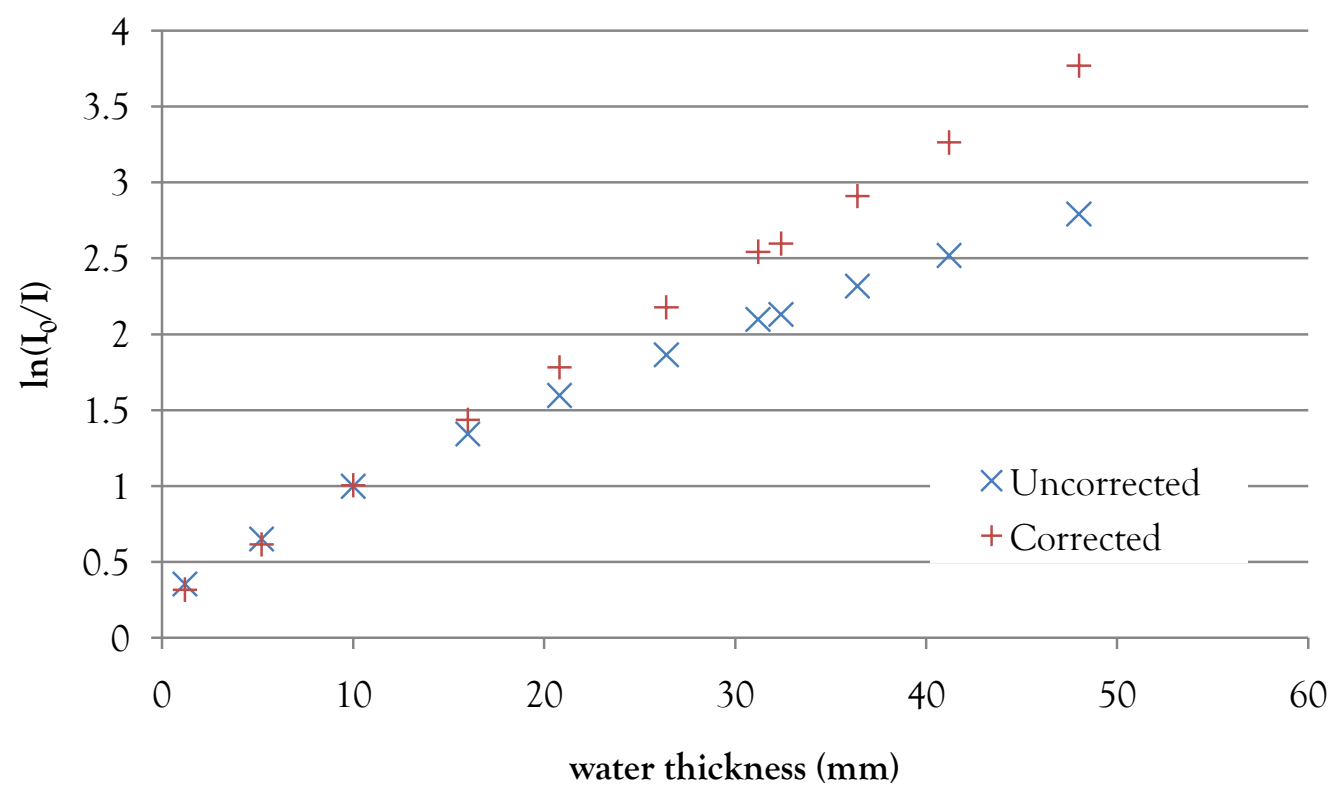

Figure 3-4. The relative intensity of a $45 \mathrm{kV}$ x-ray beam as a function of water thickness. (The offset at zero is a results of attenuation from the container and the animal bed supporting the water phantom.)

In the absence of beam-hardening, $p(\ell)$ should be linear with respect to $\ell$, with a slope equal to the initial slope of the $p(\ell)$. For beam energies of $45 \mathrm{kV}, 55 \mathrm{kV}$, and $65 \mathrm{kV}$, we measured initial slopes of $0.731 \mathrm{~cm}^{-1}, 0.642 \mathrm{~cm}^{-1}$, and $0.573 \mathrm{~cm}^{-1}$, respectively. As the thickness of the water increases, the attenuation falls below a linear extrapolation of the initial slope. Therefore, to correct for beam-hardening, we wish to determine

$$
p^{\prime}(\ell)=f(p(\ell))=\mu_{0} \ell
$$

such that $p^{\prime}(\ell)$ is linear in $\ell$. The correction was calculated by fitting a $3^{\text {rd }}$ order polynomial to the relationship between the measured attenuation and the extrapolation of the initial slope. The resulting beam hardening correction equations were determined to be: 
$45 \mathrm{kV}$ :

$$
p^{\prime}=0.8346 p+0.1656 p^{2}+0.0069 p^{3}
$$

$55 \mathrm{kV}$ :

$$
p^{\prime}=0.8260 p+0.2111 p^{2}-0.0042 p^{3}
$$

$65 \mathrm{kV}$ :

$$
p^{\prime}=0.8159 p+0.2636 p^{2}-0.0195 p^{3}
$$

Without beam hardening correction, the reconstructed intensity at the centre of a $48 \mathrm{~mm}$ diameter cylindrical water phantom scanned with the $45 \mathrm{kV}$ beam is only $70 \%$ of the intensity near the edges. With the projection data corrected for beam hardening, the cupping artefact in the reconstruction is greatly reduced, although still visible, with the center intensity $9 \%$ below the intensity near the edge (Figure 3-5). This residual cupping is likely a consequence of scatter. The small disk of reduced intensity at the center of the CT reconstruction (Figure 3-3) is the result of a non-linear detector response near the edges ${ }^{2}$.

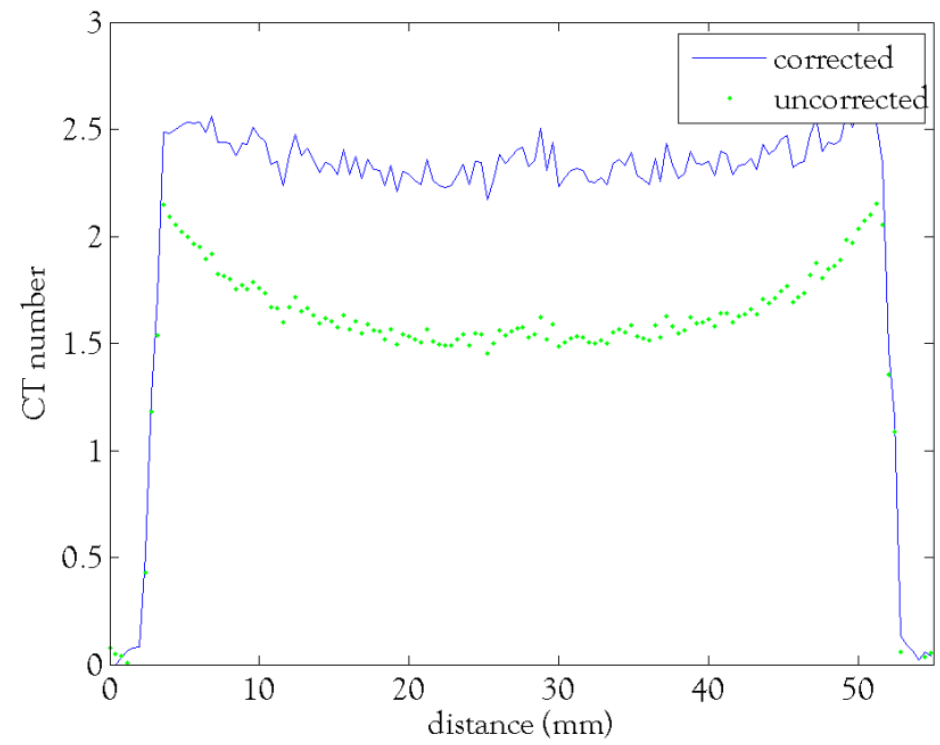

Figure 3-5. Profile through $5 \mathrm{~cm}$ diameter water phantom, reconstructed with and without beam hardening correction.

${ }^{2}$ The detector seems to be comprised of four adjacent segments with slight variances in the linearity of their response. The differences are too subtle to be visible on the projections, but can be detected by mathematical analysis of a projection. On the reconstruction the artefact is visible as a spot of depressed intensity at the center of rotation and a ring approximately half way between the center and the edge of the reconstruction. 
Because the beam-hardening correction is implemented as a polynomial scaling of the measured CT data, any noise in the CT projections is also amplified with the signal, increasing the noise in the reconstructed CT. The percent noise levels in the CT images remain considerably lower than those in the SPECT emission data and so have negligible impact on the noise in the reconstructed SPECT image.

The beam-hardening correction as derived is only correct when the attenuating medium is similar to water. For attenuating media containing higher $\mathrm{Z}$ materials, in particular bone, more sophisticated beam-hardening correction is required to obtain accurate reconstruction, typically involving an iterative approach (Hsieh, Molthen, Dawson, \& Johnson, 2000). Correction for the artefact and second order beam-hardening corrections were not implemented in our CT reconstruction because it was felt that the impact on the SPECT reconstruction would be small compared to the noise levels in the SPECT image.

\subsection{SPECT reconstruction software}

The SPECT reconstruction software is our own implementation of OS-EM reconstruction described in \$2.2.2.3-2.2.2.4 for the multiplexed multi-pinhole NanoSPECT scanner. The vendor does provide reconstruction software, but it is proprietary and locked, and therefore cannot be modified to implement attenuation correction, or scatter correction.

The reconstruction code proceeds in the following manner:

1. Calculation of attenuation maps (if attenuation correction is enabled)

2. Calculation of normalization maps

3. Initialization of the image estimate

4. Iteratively cycling through:

a. Forward-projection of current estimate

b. Addition of scatter estimate (if scatter correction is enabled) 
c. Division of the true projection data by the simulated projections

d. Backprojection of the ratio

e. Scaling the previous estimate by the backprojected ratio

f. Normalization

\subsection{Cumulative attenuation maps}

To model attenuation, a $45 \mathrm{kVp}$ cone-beam CT acquired immediately before the SPECT scan was re-sampled on a grid aligned to the SPECT reconstruction. The CT values in each voxel were scaled to obtain linear attenuation coefficients for the isotope of interest to create a custom attenuation map for each subject being imaged. The CT-to-attenuation scaling factors were determined experimentally, as described in $\$ 5.1 .3$.

This attenuation map was then converted into a cumulative attenuation map, containing a map of the total attenuation for each voxel in the reconstruction. Cumulative attenuation must be calculated for every possible combination of reconstruction voxel, projection angle, and pinhole. The cumulative attenuation for the photons originating from any particular voxel is the line integral of the linear attenuation along the path from that voxel to the detector. This was calculated numerically, by progressing though the attenuation map, starting at the voxel of interest, slice by slice towards the pinhole, summing the attenuation coefficients for each slice. The attenuation coefficient for each slice was calculated by bilinear interpolation of the closest four voxels.

\subsubsection{Normalization}

A normalization map was created for each subset, corresponding to the

$$
\sum_{i} h_{i j}
$$

term in the ML-EM algorithm. In practice, the normalization map can be created by backprojecting a projection consisting entirely 1s. However, the system matrix can be decomposed into terms 
which depend only on the pixel in the projection data, and those which depend on both the pixel and the voxel where the photon originates. In the former category are corrections for decay (since the start of the scan), and the detector sensitivity correction. In other words, using the notation previously introduced for the system matrix, where $h_{i j}$ is the probability that a photon emitted from voxel $j$ is detected in pixel $i, a_{i}$ is factor which accounts for decay and detector sensitivity, which is independent of the source voxel, and $h_{i j}^{\prime}$ accounts for photon transport (and possibly attenuation and scatter although in our software, attenuation and scatter were modelled in the reconstruction as described in $\S 3.3 .3$, not built into the system matrix):

$$
h_{i j}=a_{i} h_{i j}^{\prime}
$$

Then, the ML-EM equation becomes:

$$
\hat{f}_{j}^{(n+1)}=\hat{f}_{j}^{(n)} \frac{1}{\sum_{i} a_{i} h_{i j}^{\prime}} \sum_{i} a_{i} h_{i j}^{\prime} \frac{g_{i}}{\sum_{k} a_{i} h_{i k}^{\prime} \hat{f}_{k}^{(n)}}
$$

Since $a_{i}$ can be factored out of the forward and backprojection step, it remains only in the normalization.

$$
\hat{f}_{j}^{(n+1)}=\hat{f}_{j}^{(n)} \frac{1}{\sum_{i} a_{i} h_{i j}^{\prime}} \sum_{i} h_{i j}^{\prime} \frac{g_{i}}{\sum_{k} h_{i k}^{\prime} \hat{f}_{k}^{(n)}}
$$

Therefore, the normalization map is calculated by backprojecting the detector sensitivity maps, scaled by the decay correction into the reconstruction space.

Since the projection data are adjusted by the nanoSPECT software at the time of acquisition to correct for irregularities in the detector sensitivity, our reconstruction code assumes uniform detector sensitivity, although it could be readily adapted to incorporate the true detector sensitivity maps with projection data uncorrected for non-uniformity in the detectors. 
Decay of the isotope used for imaging is modelled in the system matrix by:

$$
a_{i}=0.5^{t_{p} / t_{h}}
$$

where, $t_{p}$ is the delay between the start of the first projection and the start of the current projection set during which pixel $i$ was acquired, and $t_{h}$ is the half-life of the isotope in question. This results in a reconstruction that has been decay-corrected to the time of the first projection. For short scans with long lived isotopes, decay is a relatively minor concern, but when the duration of the scan is significant relative to the half-life of the isotope, decay will have a clear effect on the reconstruction. For a 30 minute acquisition of a ${ }^{99 \mathrm{~m}} \mathrm{Tc}$ study, the activity decreases by approximately $5.5 \%$ between the first set of projections acquired and the last. (Modelling the decay in this way, rather than precorrecting the projections should, in theory, result in more accurate reconstruction, since the maximum likelihood algorithms assume Poisson noise. When Poisson data are scaled, the uncertainty on the scaled variable no longer follows a Poisson distribution, which compromises the accuracy of the reconstruction.)

\subsubsection{Forward- and back-projection}

In the forward projection step, the current estimate of the source distribution is rotated and shifted in the z-direction, since the system matrix is only calculated for a single projection angle. If attenuation correction is enabled, the source is then scaled by the pre-calculated cumulative attenuation map for each pinhole. The forward projector than 'projects' this result using the precalculated system matrix look-up table (\$3.4) and adds the PSF corresponding to each voxel of the current reconstruction estimate to the projection.

If dual-energy-window scatter correction is enabled, scatter was estimated by spatially smoothing the data in the downscatter window $\left(97-125 \mathrm{keV}\right.$ for $\left.{ }^{99 \mathrm{~m}} \mathrm{Tc}\right)$ with a Gaussian filter (FWHM $=3.0 \mathrm{~mm}$ ), 
scaling it by the specified scaling factor, $\mathrm{k}$, and adding the smoothed and scaled downscatter projections to the forward projections during each forward projection step of the OS-EM iterations.

The true projections are then divided by the forward projection of the current estimate. The trueto-estimated ratio is then backprojected using the same system matrix look-up table and scaled by the cumulative attenuation map. This backprojection is normalized, and the previous estimate is scaled by the normalized backprojection.

At each step of the iterative reconstruction, the projections and reconstruction must be shifted slightly to account for any mechanical misalignments in the SPECT camera, acquired when the camera is calibrated and stored in the header of the projection data file.

\subsubsection{OS-EM Subsets}

Although OS-EM dramatically speeds up convergence compared to ML-EM, we want to avoid any possible dependence of the final image on the number or order of subsets. Therefore, in our OSEM implementation, we reduce the number of subsets as the reconstruction progresses as proposed by the original paper by (Hudson \& Larkin, 1994). For the final set of iterations, we use only a single subset, at which point the reconstruction becomes ML-EM. The default subset scheme in our code is 8 iterations with 6 subsets, followed by 8 iterations with 4 subsets, 8 with 3, and finally 8 iterations with all the projections.

\subsection{The system matrix}

A system matrix which accurately models the SPECT camera is essential for accurate reconstruction. At a minimum, the system matrix must model the photon transport of unscattered photons from the source to the detector. For more accurate reconstruction, the system matrix should also model photon attenuation, scatter, and the intrinsic resolution of the detector. 
Although the full system matrix is enormous, it is also relatively sparse, allowing the system matrix to be stored as a table of pre-calculated point spread functions.

The point spread function (PSF) is the signal observed on the detector when a point source is placed within the field of view of the SPECT camera. Because of the finite size of the pinhole, photon penetration at the edge of the pinhole, and the limited intrinsic resolution of the detector, even an ideal point source results in a projection on the detector which resembles a blurred ellipse. The shape and size of the PSF varies with source position. An accurate model of the PSF is essential for optical contrast, resolution, and quantitative accuracy.

The pinholes in most pinhole collimators consist of back-to-back cones drilled through a solid slab of attenuating material. Near the edge of the pinholes, the material is thin enough to allow some photons to penetrate, resulting in a widening of the PSF. The degree to which the PSF spreads out due to edge penetration depends on the photon energy - higher energy photons are more penetrating and will have larger PSFs - the material used to construct the attenuator, and the opening angle of the pinhole. Larger opening angles allow for a larger field of view with a single pinhole, but at the cost of poorer resolution due to edge penetration.

The PSF can be acquired in several ways. Analytic modelling of the PSF is one option. An analytic calculation of the point spread function for an ideal pinhole (diameter $=0 \mathrm{~mm}$ ), and with modelling of aperture penetration, is available in (Smith \& Jaszczak, 1998). More detailed analytic models of the PSF for more realistic right-circular double cone and oblique-circular double cone pinholes have been derived by (Bal \& Acton, 2006). Although accurate, the high number of parameters and complexity of the analytic PSF model are prohibitive for on-the-fly calculations during reconstruction. 
The second possibility is direct measurement, where a point source is mounted on a movable stage and stepped through each position within in the field of view. However, this method is timeconsuming and requires a setup capable of scanning a point source through the field of view with a high degree of mechanical precision.

The PSF can also be calculated using ray tracing to determine the thickness of collimator between the source and the detector location. (Schramm, Ebel, Engeland, Schurrat, Béhé, \& Behr, 2003) (Funk, Després, Barber, Shah, \& Hasegawa, 2006) (Feng, et al., 2010)

Finally, the point spread function can also be derived by Monte Carlo simulation. In Monte Carlo simulation a model of the source, pinhole, and detector are modeled in photon transport modelling software such as EGS or GEANT. Monte Carlo has the advantage of being able to model scatter, however modelling a sufficient number of events to obtain effectively noise free PSF data can be extremely time-consuming. Variance reduction such as forced detection can be used to speed up Monte Carlo generation of the PSF (Gieles, de Jong, \& Beekman, 2002).

This system matrix in our reconstruction software was generated by ray-tracing a digital model of the multi-pinhole collimator used in the nanoSPECT/CT system. To improve accuracy, we subdivided each voxel into sub-voxels and each pixel into sub-pixels as in (Feng, et al., 2010). Our system matrix was created by subdividing the voxels into $8(2 \times 2 \times 2)$ sub-voxels, and dividing the pixels into $100(10 \times 10)$ sub-pixels. The software then iterated through each sub-pixel and sub-voxel to determine if the ray joining the two passed through the pinhole. For each ray, the intersections between the ray and the collimator were calculated analytically. The intersections of the back-toback cones comprising the pinhole with each other and with and the front and back surfaces of the aperture plate define three ellipses. To determine if a ray reaches the detector, the algorithm tests if it falls within all three. The weighting factor for each voxel-pixel pair is then calculated as: 


$$
\frac{\text { total number of rays which pass through the collimator }}{\text { number of sub-pixels } \times \text { number of sub-voxels }} \times\left(\frac{d_{0}}{d}\right)^{2} \times \cos \theta
$$

where $d$ is the distance from the voxel to the pixel, $d_{0}$ is the distance from the central voxel to the central pixel, and $\theta$ is the angle between the ray and the normal of the detector.

Although the system matrix generation software was written to be capable of modelling pin-hole edge penetration, the two cones defining the pinholes of the APT2 apertures meet at a relatively shallow angle and edge penetration was found to be negligible for ${ }^{99 \mathrm{~m}} \mathrm{Tc}$ photons.

To speed up reconstruction, the system matrix in the software used in this work was pre-calculated and stored as a table. Each row contains a reconstruction voxel-projection pixel pair with the weighting factor which corresponds to the (normalized) probability that a photon emitted in the voxel will be detected in the detector pixel. Calculating the system matrix on a PC with an Intel Core2 Quad CPU @ 2.66GHz took approximately three days, though optimization of the pinhole modelling code could likely reduce this significantly. The storage space required for the full system matrix required to reconstruct an image with $(0.6 \mathrm{~mm})^{3}$ voxels was $1.72 \mathrm{~GB}$.

The system matrix was only calculated for each pinhole and for a single projection angle (nominally $0^{\circ}$ ). All other projection angles were handled by appropriate rotations and shifts of the data prior to or following the projection or backprojection step.

\subsection{Maximum object size with multiplexed projections}

Being able to accurately reconstruct an image from a set of projections depends on certain conditions which specify the minimal projection information required for reconstruction. In the non-multiplexed cone-beam imaging case, the conditions are those specified by Tuy for transmission imaging (\$2.2.1.1). The situation is less clear in the case of multiplexed projections, since some of the information is now ambiguous where the projections overlap, and we are 
unaware of any analytical work which describes the exact conditions under which multiplexed cone beam projections, as is the case with the nanoSPECT, can be unambiguously reconstructed.

The literature regarding multiplexing in pinhole SPECT is somewhat sparse and deals mostly with specific example cases. Because of the large number of possible multiplexing configurations, scanner geometries, and different imaging tasks, it is difficult to provide a general answer to the question of how much multiplexing is possible before the degradation in image quality begins to outweigh the gains in efficiency. It seems likely that the optimal number of pinholes and degree of multiplexing is task-dependant, and strongly influenced by the size of the object being imaged, the specificity of the tracer, and the expected distribution and intensity of background activity.

(Cao, Bal, Accorsi, \& Acton, 2005), using digital cold rod and mouse brain phantoms and Monte Carlo simulation, investigated the optimal number of pinholes for mouse brain imaging. They demonstrated that convergence was slowed and bias increased as the number of pinholes increased. For the geometry they investigated, they concluded that 9 is the optimum number of pinholes, beyond which no improvement was observed in the reconstructed image. Similar conclusions were reached by (Mok, Wang, \& Tsui, 2009) with simulations of full-body mouse imaging and a digitized hot-sphere phantom. They also found that up to a certain point, bias increased while noise statistics improved slightly with higher degrees of multiplexing. At a degree of multiplexing of about $40 \%$, the bias-noise trade-off reached an optimum and degraded with further multiplexing.

As well as the collimator geometry, the degree of multiplexing also depends on the activity distribution. Sparse activity distributions may benefit from the additional efficiency afforded by multiplexed projections, while non-sparse distributions produce artefacts in the reconstructions as a result of projection overlap (Vunckx, Suetens, \& Nuyts, 2008) (Mok, Tsui, \& Beekman, 2011). 
The specific conclusions of all of the studies just mentioned are difficult to apply directly to the nanoSPECT imaging system, since none of them simulate the particular geometry of that scanner. Therefore, we chose to investigate empirically the maximum source distribution volume that could be reconstructed without noticeable artefacts. We can observe, simply by attempting to scan a cylindrical phantom (50 $\mathrm{mm}$ diameter), that a large, uniform source cannot be correctly reconstructed (Figure 3-6).
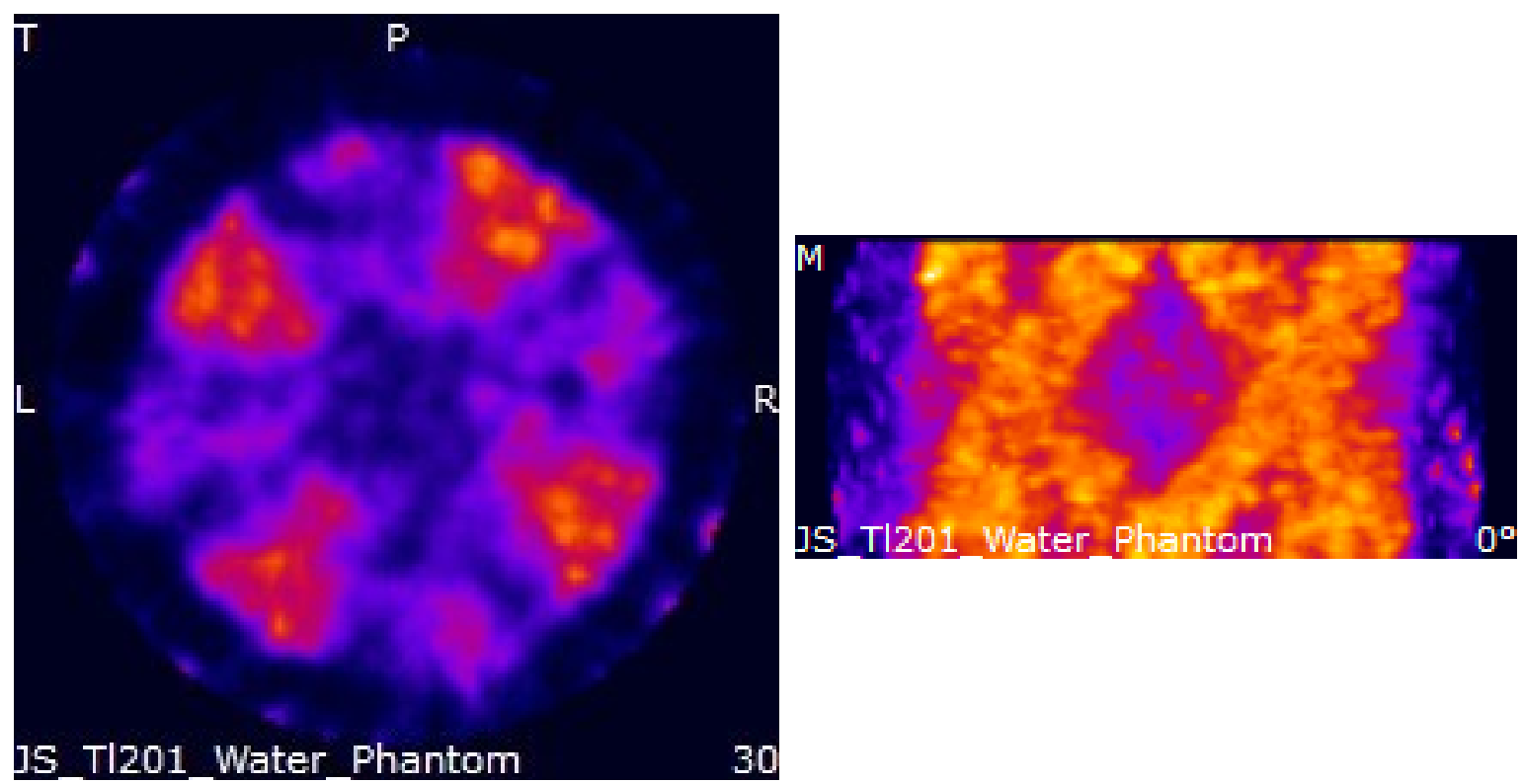

Figure 3-6. Reconstruction of a $50 \mathrm{~mm}$ diameter cylindrical phantom showing the artifacts when the source distribution is too large. (left) Transverse view, (right) maximum intensity projection. (Reconstructed using vendor-supplied software.)

To determine the maximum object size which could be reconstructed, we created a series of cylindrical digital phantoms with diameters from $10-50 \mathrm{~mm}$, increasing in $5 \mathrm{~mm}$ steps. Simulated projections were created by forward projecting the phantoms, and Poisson noise was simulated and added to the projections. The activity level in the projections was equivalent to that obtained experimentally in a 30 minute scan of a phantom with an activity concentration of approximately $1 \mathrm{MBq} / \mathrm{mL}$. The projections were then reconstructed with our reconstruction code. This was done without attenuation modelling. 
The experiment was also carried out with the nanoSPECT scanner and physical cylindrical phantoms with diameters of $9 \mathrm{~mm}, 27 \mathrm{~mm}$, and $37 \mathrm{~mm}$. The phantoms were filled with solutions of ${ }^{99 \mathrm{~m}} \mathrm{Tc}$, and scanned for $30 \mathrm{~min}: 48$ projections, 150 s per projection. The scan followed a helical trajectory with an axial length of $25 \mathrm{~mm}$.

\subsection{Digital phantom results}

Reconstructions of some of the digital phantoms are shown in Figure 3-7. For the phantoms smaller than $35 \mathrm{~mm}$, reconstruction successfully recovers the original phantom. The only noticeable artefact, other than noise, is some 'ringing' at the edges of the phantom. When the diameter of the digital phantom exceeds $35 \mathrm{~mm}$, subtle helical artefacts begin to appear in the reconstruction towards the outside edge of the phantom. (These are difficult to see on the cross sections through the reconstruction, but are slightly visible in the maximum intensity projection and show up clearly when scanning through the stack of cross-sectional views.) For the phantoms of $40 \mathrm{~mm}$ and larger, circular artefacts are visible in the transverse slices of the reconstruction. The reconstructions of the 40,45 , and $50 \mathrm{~mm}$ diameter phantoms also contain activity in the regions outside of the phantom.

Individual voxel values exhibited considerable noise, although the average voxel value in the reconstruction is close to that of the original phantom. The activity distribution of pixel values within the phantom was Gaussian, with the largest standard deviation in the smallest phantom, where a larger proportion of the pixels were near the edge of the phantom than in larger phantoms.

If a region of interest $(\mathrm{ROI})$ larger than the phantom is used for quantification, the total activity in the reconstructed phantom differed from the original phantom data by less than $0.1 \%$ for the phantoms smaller than $35 \mathrm{~mm}$. For the $40-50 \mathrm{~mm}$ phantoms the total activity in the reconstruction was higher than in the phantom. Restricting the ROI to include only the voxels of 
the original phantom, the total activity was between $96-98 \%$ of truth for the $10-35 \mathrm{~mm}$ phantoms. For the larger phantoms, the activity in the reconstructed phantoms was 95\%, 93\% and $90 \%$ of the true value.
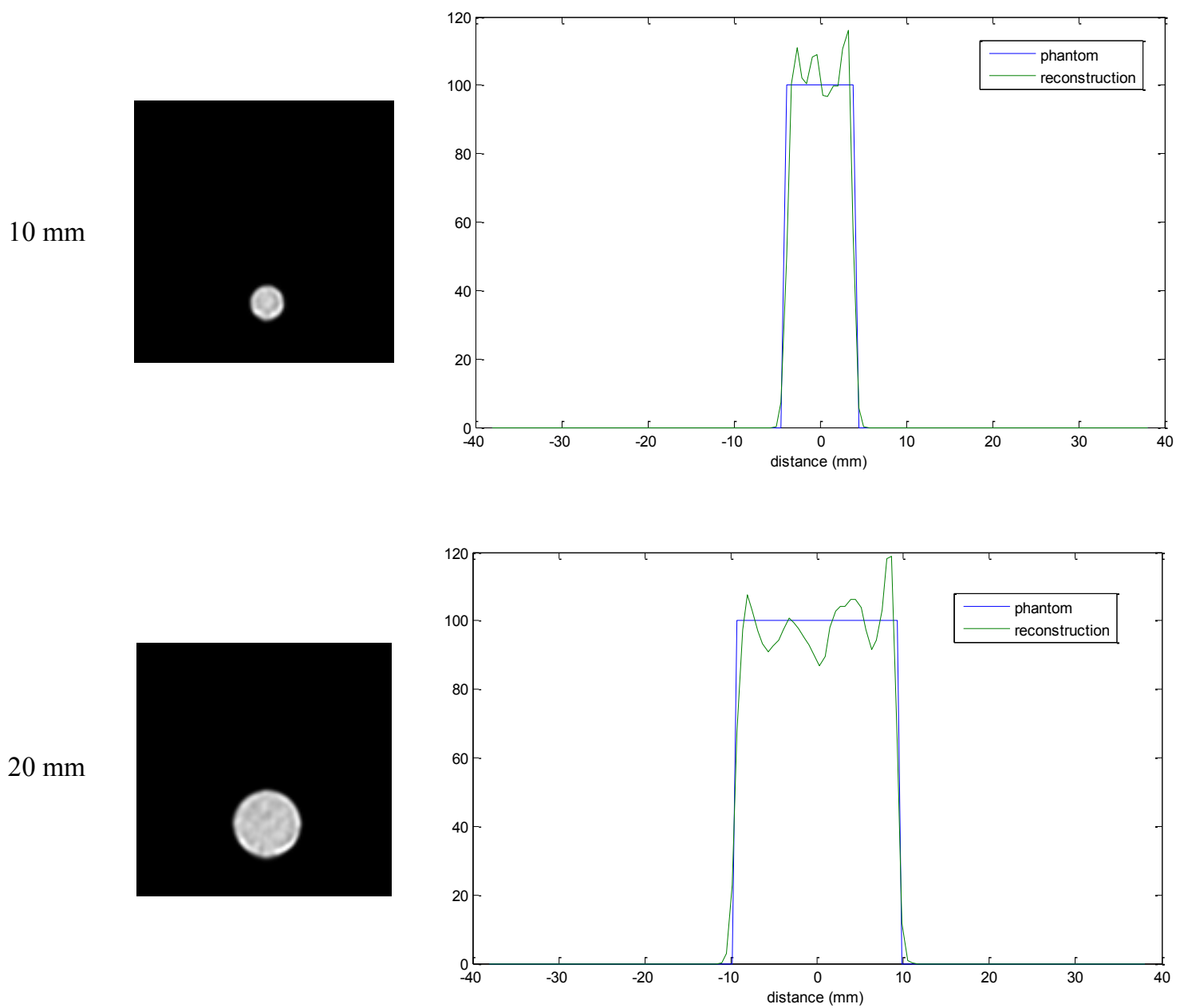

Figure 3-7a. Reconstructions of cylindrical phantoms (left) and profiles through the middle of the central slice (right). The vertical scale shows nominal activity, set to 100 for the phantoms. 

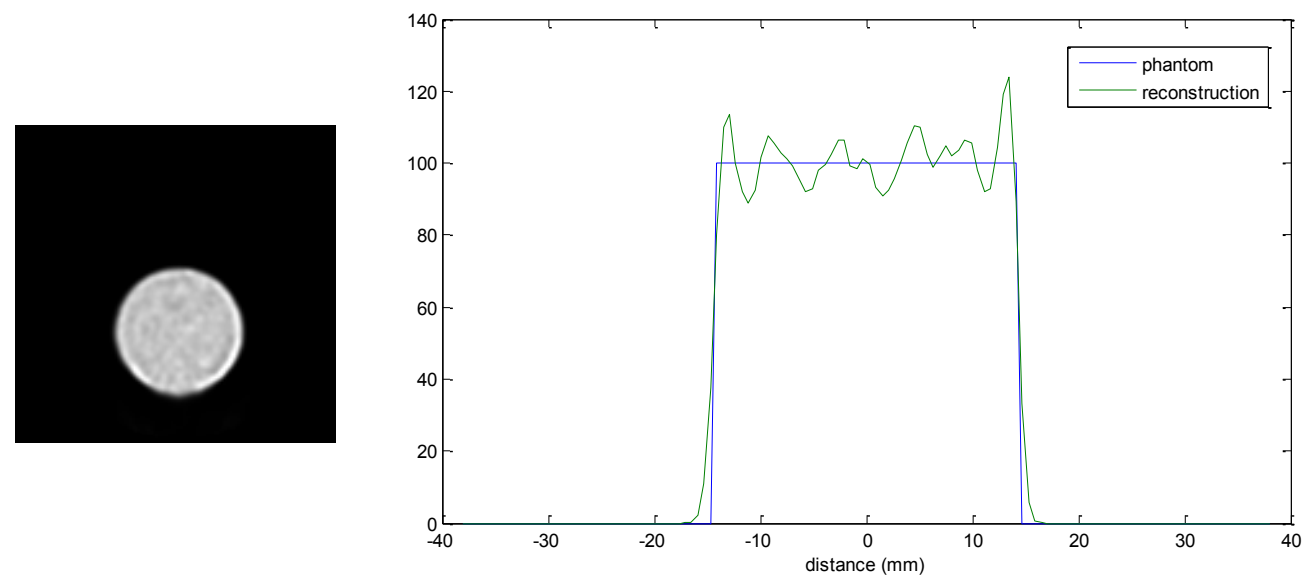

$40 \mathrm{~mm}$
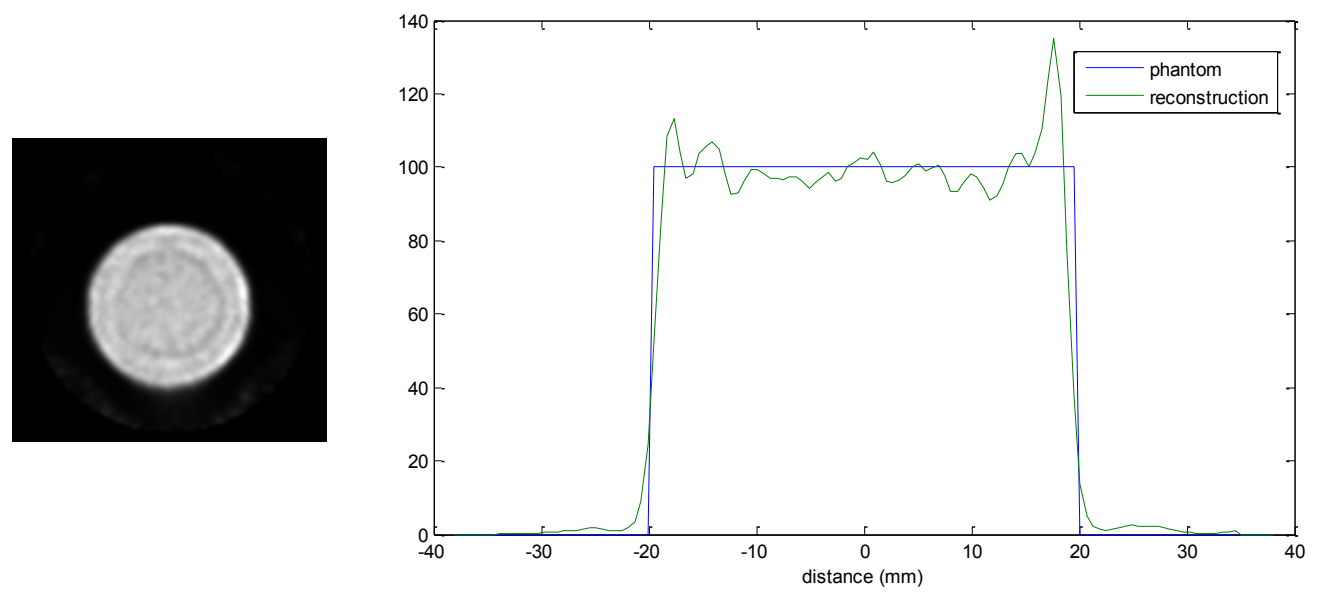

$50 \mathrm{~mm}$
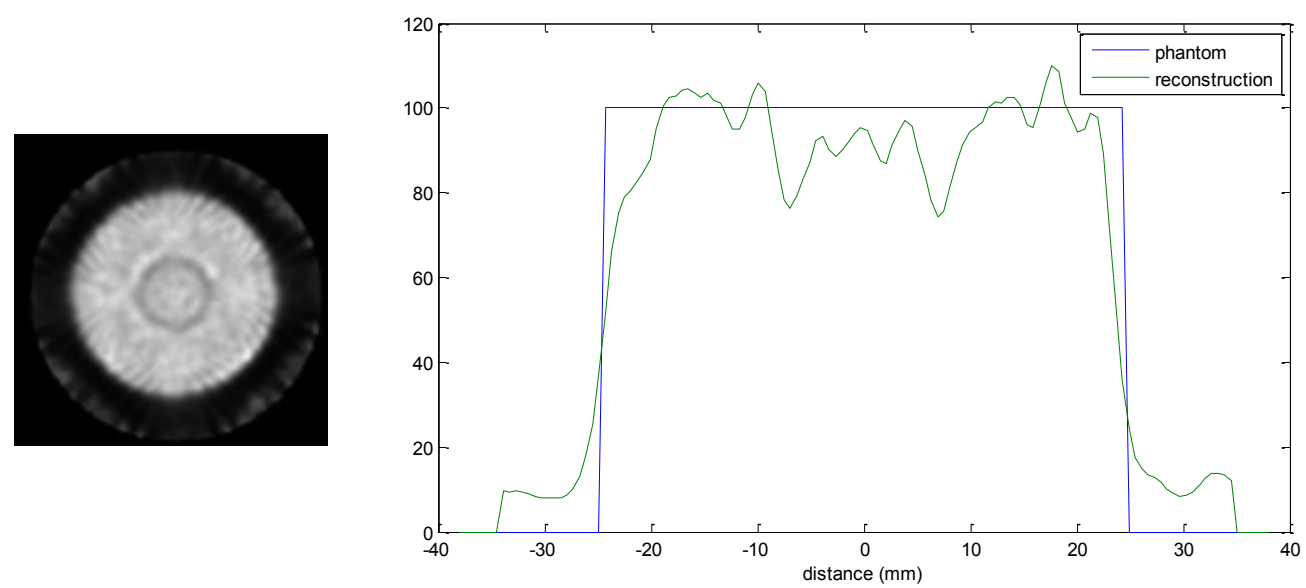

Figure 3-7b. Reconstructions of cylindrical phantoms (left) and profiles through the middle of the central slice (right). The vertical scale shows nominal activity, set to 100 for the phantoms. 
Since the projections in this case were simulated, the system matrix to create the projections exactly matched that used to reconstruct the images. Therefore, any artefacts arise from the imaging geometry itself and not from a mismatch between the system matrix used for reconstruction and the physical imaging geometry.

\subsection{2 ${ }^{99 \mathrm{~m}} \mathrm{Tc}$ phantom results}

Figure 3-8 shows the reconstruction of the ${ }^{99 \mathrm{~m}} \mathrm{Tc}$ phantoms. The 9 and $23 \mathrm{~mm}$ phantoms reconstructed are essentially uniform apart from the noise, but subtle helical artefacts are visible in the $37 \mathrm{~mm}$ phantom.

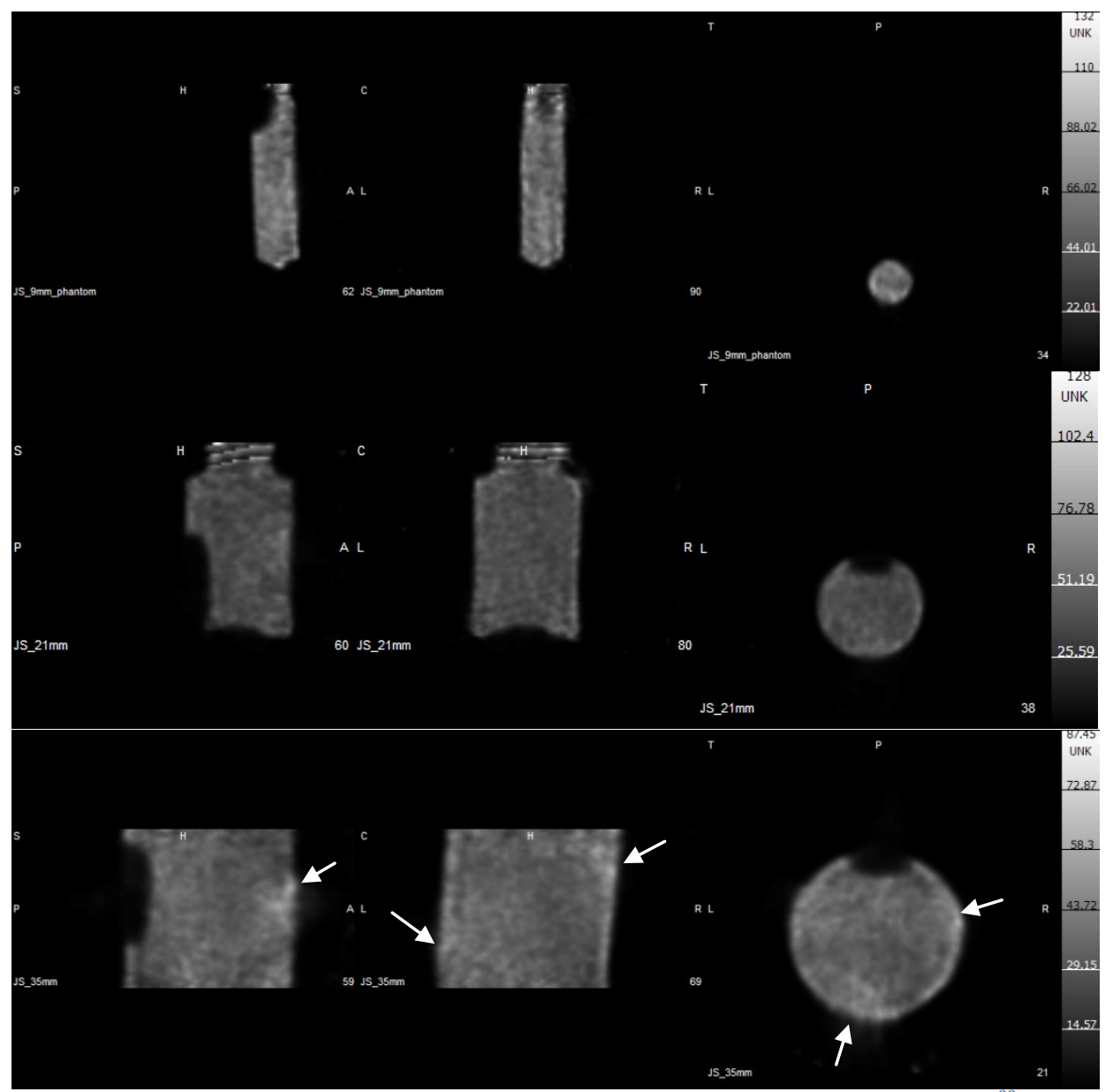

Figure 3-8. Reconstructions of $9 \mathrm{~mm}, 27 \mathrm{~mm}$, and $37 \mathrm{~mm}$ phantoms containing ${ }^{99 \mathrm{~m}}$ Tc are shown in the top, middle, and bottom rows respectively. Artefacts in the $37 \mathrm{~mm}$ phantom are visible but subtle. Note bright spots near the edge of the $37 \mathrm{~mm}$ phantom. In the centre image (Sagittal slice) of the $37 \mathrm{~mm}$ phantom, the intensity in upper right and lower left corners is higher than elsewhere. 


\subsubsection{Validation of $\mathrm{VOI}$ for measuring activity in small volumes}

Because of the limited resolution of the SPECT imaging system, edges in the reconstructed images are blurred. In addition, resolution recovery, which improves the resolution of the reconstructed image by accurately modelling the pinhole, also results in Gibbs 'ringing' wherever there are sharp discontinuities in activity concentration. This is evident particularly in simulations using digital phantoms where all imperfections (i.e. misalignments of the camera heads, subtle changing in the centre of rotation, etc.) are eliminated and discontinuities in the phantom are sharp. As shown in Figure 3-7 the simulated digital phantom reconstructions all exhibit a ring around the edge of the phantom where the activity is overestimated. The phenomenon is visible, but less apparent in real scans where partial volume effects and mechanical misalignments introduce some inherent smoothing. The worst case scenario occurs in the smallest phantoms where the voxels near the edge of the phantom make up a larger fraction of the total volume of interest. However, even with the ringing at the edge of the reconstruction, a the average activity measured in a VOI with a diameter of between $7-8 \mathrm{~mm}$ placed on a reconstruction of a $10 \mathrm{~mm}$ diameter phantom was within $1 \%$ of the original activity concentration. Shrinking the VOI to $6 \mathrm{~mm}$ or enlarging it to $9 \mathrm{~mm}$ resulted in quantification within $2 \%$ of truth. For larger phantoms, keeping $2-3 \mathrm{~mm}$ between the edge of the phantom and the VOI recovers the original activity with less than $0.5 \%$ error. When there is no background activity and the volume of the activity distribution can be accurately determined from the dimensions of the phantom, the activity concentration can be also be measured by extending the VOI beyond the edges of the phantom, though this is less accurate in vivo where there is nearly always some background activity in adjacent organs and tissues and measuring the volume of a specific organ is more difficult. 


\subsection{Other equipment}

\subsection{Dose calibrator}

All activity measurements were done in a Capintec CRC-25R dose calibrator. The CRC-25R is a deep-well ionization chamber, with a manufacturer quoted accuracy and linearity of $\pm 2 \%$ and repeatability of $1 \%$. The dose calibrator has a maximum resolution of $0.01 \mu \mathrm{Ci}$. The depth dependence of the dose measurement was characterized by measuring the activity of a vial containing a $1.9 \mathrm{mCi}$ drop of ${ }^{99 \mathrm{~m}} \mathrm{Tc}$-pertechnetate at varying depth from the bottom of the well chamber; the results are shown in Figure 3-9.

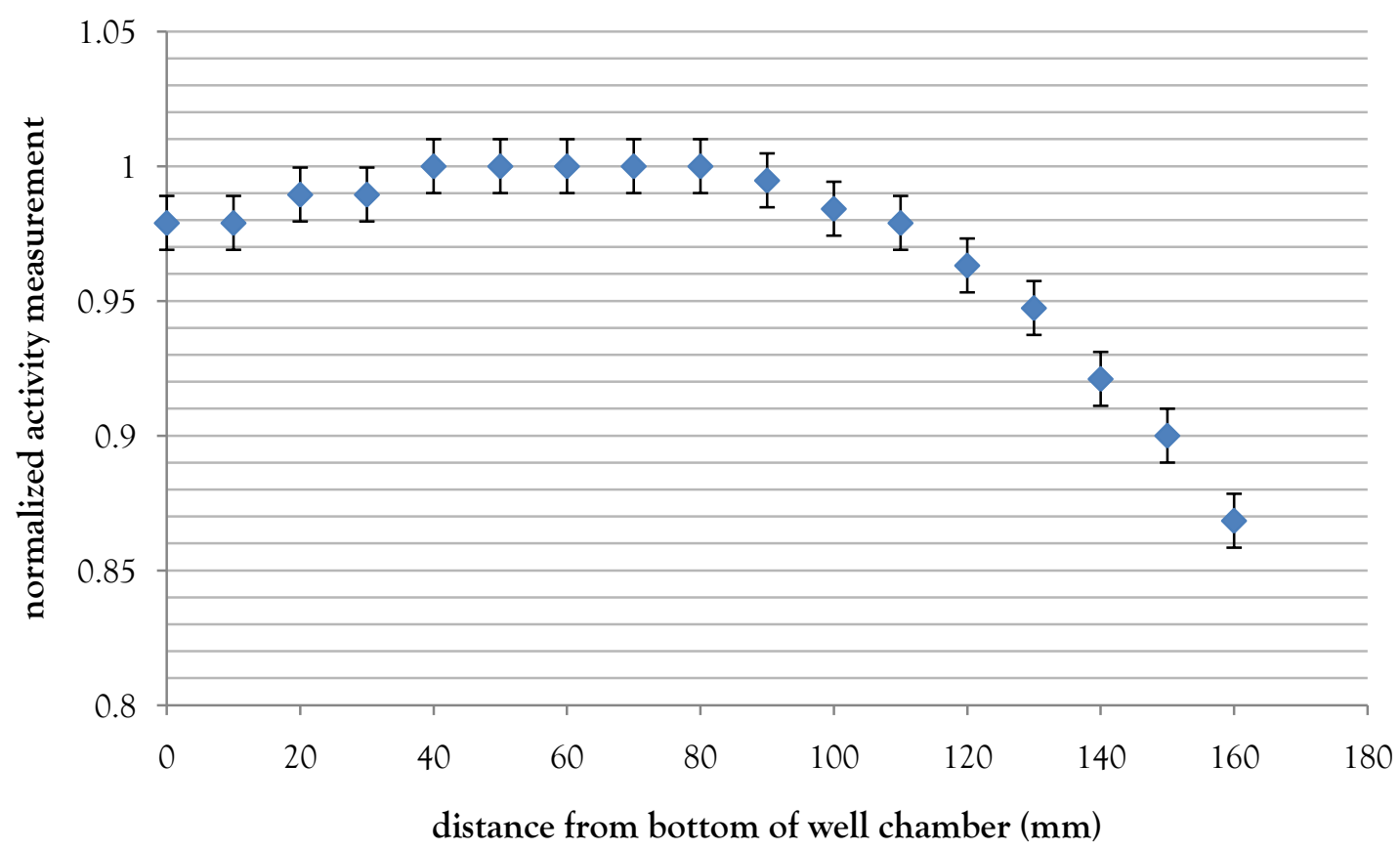

Figure 3-9. Depth dependence of Capintec CRC-25R dose calibrator measured with ${ }^{99 m}$ Tc. Activity measurements are normalized to the peak activity measurement.

From the bottom of well to a depth of $10 \mathrm{~cm}$ from the bottom, the maximum deviation of the well chamber sensitivity is about $2 \%$, with no change in sensitivity between about 4 and $8 \mathrm{~cm}$. For most of the measurements done for this work, the activity was measured in syringes with the activity 
between $80-90 \mathrm{~mm}$ from the bottom of the well, where the response is within $1 \%$ of the normalized maximum.

The sensitivity of the dose calibrator is slightly higher near the walls than in the centre. The reported activity for a source measured near the edge was 3.3\% higher than the same source measured at the centre.

Self-attenuation of the sources measured in the dose calibrator adds an additional source of error, with Compton scatter accounting for most of the attenuation of the primary photons. Moreover, the sensitivity of the dose calibrator increases with decreasing photon energy. Thus, significant scattering can result in lower energy scattered photons, increasing the reading of the dose calibrator. The activity measured for a small vial of tracer can be shown to actually increase when placed inside a water phantom in the dose calibrator. The differing amounts of self-attenuation and scatter due to variations in the geometry and dilution of the sources measured in the dose calibrator contribute to an additional approximately $1 \%$ uncertainty in activity measurements.

Obtaining reproducible measurements in the $\mu \mathrm{Ci}$ range is challenging, since the background measurement can drift by more than $1 \mu \mathrm{Ci}$ over a few minutes.

Total uncertainty of activity measurements made in the dose calibrator is estimated to be approximately $3 \%$. 


\section{Reproducibility of SPECT cardiac measurements in rats}

This chapter, in a slightly modified form has been published as Strydhorst J, Leenen F, Ruddy TD, and Wells $R G$, "Reproducibility of serial left ventricle perfusion, volume, and ejection fraction measurements using multiplexed multipinhole SPECT in healthy rats and rats after myocardial infarction." J Nucl Med 52(2011):1285-1292.

\section{I Introduction}

Before using microSPECT data for the investigation of rat models of cardiac disease, a baseline of normal data along with an assessment of measurement reproducibility is a vital prerequisite. A normal database of LV perfusion, volume, and motion has been reported for mice, (Constantinesco, Choquet, Monassier, Israel-Jost, \& Mertz, 2005) but we are not aware of similar data being available for rats.

The test-retest reproducibility of single-pinhole clinical camera SPECT measurements of LV function in rats has been reported by (Vanhove, Lahoutte, Defrise, Bossuyt, \& Franken, 2005). Measurements on healthy rats performed one week apart showed no significant differences in ejection fraction and LV volumes. Modern microSPECT systems now use multiple heads and 
multiple pinholes to obtain cardiac SPECT studies in reasonable scan times with considerably reduced injected activity.

The objective of this study is to determine the intra-subject and inter-subject reproducibility of serial measurements of perfusion, myocardial defect severity, ventricle volume and ejection fraction, using ${ }^{99 \mathrm{~m}}$ Tc-tetrofosmin and a multiplexed multi-pinhole small-animal SPECT scanner in normal rats and following myocardial infarction

\subsection{Methods}

\subsection{Scan protocol}

A total of twenty male Sprague-Dawley rats were included in this study. Eight rats were given a myocardial infarction (MI) by surgical occlusion of the left anterior descending artery four weeks before the initial scan. Healthy rats $(n=8)$ were 12 weeks of age at the start of the study with a mean initial weight of $245 \pm 15 \mathrm{~g}$. The MI rats $(\mathrm{n}=8)$ were 16 weeks of age at the start of the study with mean initial weight of $432 \pm 59 \mathrm{~g}$. The normal perfusion database was created using the data from the eight normal rats, plus an additional four normal rats, also of the same strain, age, and sex. The twelve normal rats had a mean initial weight of $254 \pm 18 \mathrm{~g}$. The study was approved by the Animal Care Ethics Committee of the University of Ottawa.

Rats were anaesthetized with $2-3 \%$ isoflorane and injected via tail vein with $2.1 \pm 0.4 \mathrm{mCi}$ of ${ }^{99 \mathrm{~m}} \mathrm{Tc}$ tetrofosmin. All scans were performed with the rat in a supine position, similar to the position used clinically (Figure 4-1).

All images were acquired with the NanoSPECT small-animal camera (Bioscan, Washington, DC). This camera employs a multiplexed, multi-pinhole configuration with four detector heads, which allows a full $360^{\circ}$ of projection data to be acquired by a $90^{\circ}$ rotation of the gantry. Translation of the rat through the bore of the scanner as the projections were acquired resulted in a helical 
scanning pattern. A total of 48 projections were acquired, equally spaced around the rat. The pinhole collimator in front of each detector head had a grid of nine $2 \mathrm{~mm}$ focusing pinholes. Although this configuration resulted in some ambiguity at the detector due to overlapping projections from different pinholes, this was dealt with in the vendor-supplied software by appropriate design of the reconstruction algorithm (Schramm, Ebel, Engeland, Schurrat, Béhé, \& Behr, 2003).

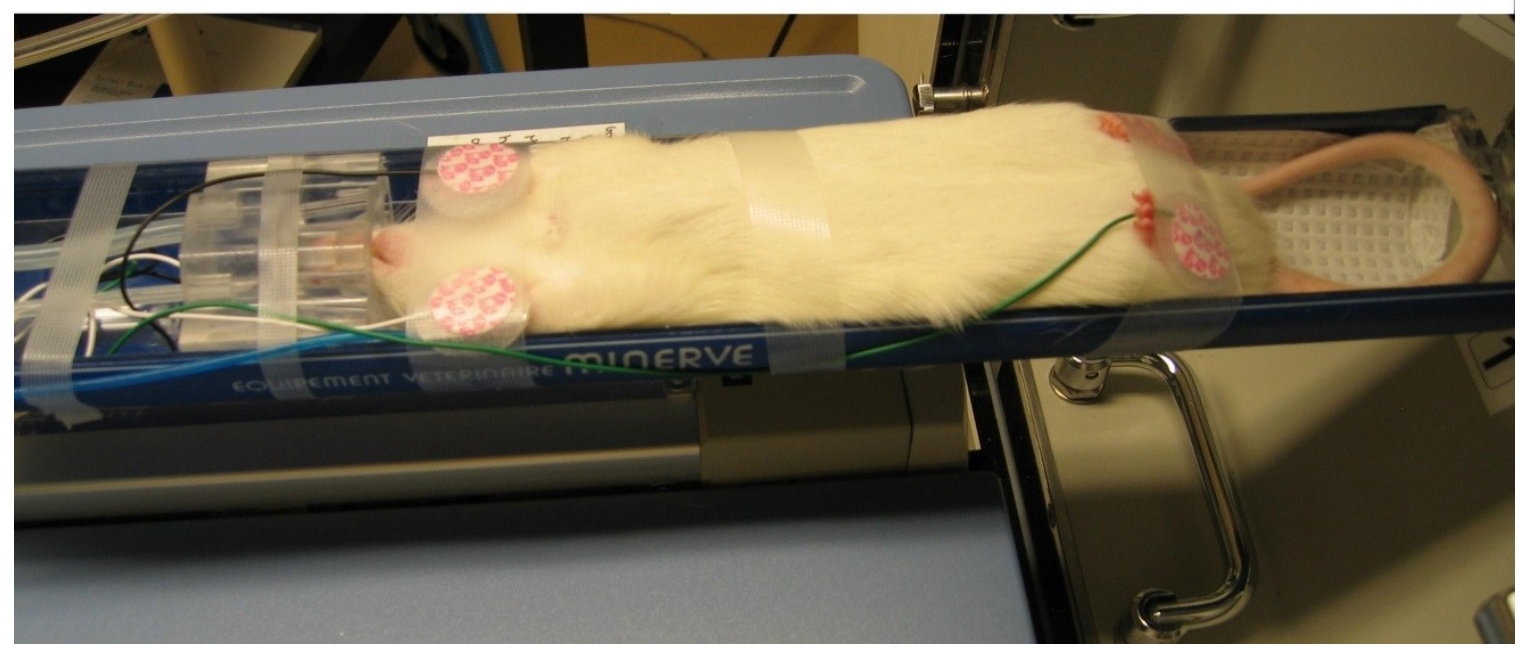

Figure 4-1. Rat positioned on animal bed with ECG leads attached to its paws.

The temperature of the rat was maintained by a heated Minerve animal bed (Bioscan, Washington, DC) maintained between $34^{\circ} \mathrm{C}$ and $35^{\circ} \mathrm{C}$. To avoid interference from the probe, the temperature of the rat was not directly monitored during the scans. We have previously measured the relationship between the bed temperature and the rat body temperature. The rat body temperature was found to be stable at $35.4 \pm 0.9^{\circ} \mathrm{C}$ for bed temperatures over the range of $33^{\circ} \mathrm{C}$ to $36^{\circ} \mathrm{C}$.

A small animal monitoring and gating system (Model 1025T, SA Instruments, Stony Brook, NY), was used to monitor the breathing and heart rate of the rat. Breathing was detected during the scan by a pressure sensitive pad placed under the rat. The heart rates of the rats were monitored during 
the scan by three ECG leads. Initial scans were done with adhesive neonatal ECG electrodes attached to the paws; subcutaneous ECG leads were found to provide a more reliable ECG trace and used for later scans. The rats were anaesthetized during placement of the leads and no discomfort arising from the use of subcutaneous leads was evident. The data were gated by dividing the R-R interval of the ECG into 8 segments or equal duration and assigning each count detected to a frame corresponding to its position in the cardiac cycle. The nominal R-R interval was set to an initial value, typically in the range 150 - $200 \mathrm{~ms}$ (equivalent to 300 - $400 \mathrm{bpm}$ ). Data collected during beats with an R-R interval that differed from the nominal interval by more than $9 \%$ were discarded. In the case that the heart rate drifted during the acquisition, the nominal R-R interval was automatically adjusted up or down by the software.

Based on previous work done in our lab that measured the dependence of reproducibility on acquisition duration, a total scan duration of 30 minutes was chosen for all studies. Scans of the healthy rats were performed in 10 minute segments beginning 30, 40, 50, 60, 70, and 80 minutes post tracer injection and the projection data from the three 10 minute time segments beginning at 50,60 , and 70 minutes post-injection were summed prior to reconstruction, resulting in a total of 7-12 million counts used for each reconstruction. Scans of the infarcted rats were performed as a single 30 minute data acquisition commencing 30 minutes post-injection, though the protocol was otherwise identical. Some of the early scans of the normal rats were missing the data from the 30 and 40 minute post-injection time frame. As biodistribution of tetrofosmin is very stable after 30 min, we do not expect there to be an appreciable difference between those scans started 30 minutes post-injection and those starting 50 minutes post injection.

The healthy rats were scanned once per week for five weeks. The MI rats were scanned twice per week, also for a total of five scans over 15 days starting 4 weeks post infarction. The timing of the 
MI scans post-surgery was chosen to minimize changes due to further remodelling or healing during the course of the 5 imaging sessions. Previous work has shown that the infarct size in the rat stabilizes by approximately 3 weeks post surgery (Fishbein, Maclean, \& Maroko, 1978).

Projection data were reconstructed using the vendor software supplied with the NanoSPECT system. Reconstruction parameters were: 3D Gaussian filter between iterations with a full-width half-max of $0.63 \mathrm{~mm}$ ('high' smoothing), a voxel size of $0.6 \mathrm{~mm} \times 0.6 \mathrm{~mm} \times 0.6 \mathrm{~mm}$, and 24 iterations ( 8 iterations of 6 subsets, 8 iterations of 4 subsets, and 8 iterations of 3 subsets; 'high' setting).

The reconstructed SPECT scans were analyzed with 4DM-SPECT (Invia Medical Solutions, Ann Arbor, MI), to measure perfusion, ungated LV volume, LV ejection fraction (LVEF) and the volumes of the LV at systole and diastole. To analyze a rat-sized heart using 4D-MSPECT software, the voxel dimensions were relabelled to be $6 \mathrm{~mm} \times 6 \mathrm{~mm} \times 6 \mathrm{~mm}$ to obtain an approximately human-sized heart. Within 4DM-SPECT, the heart was oriented and the valve plane specified by the user. For consistency, the location of the valve plane was determined by the location of the edge of the inferior wall of the myocardium in the horizontal short axis From the ungated data, 4DMSPECT calculated the ungated LV volumes and created a perfusion map for each scan. The perfusion map was normalized with respect to the point of maximum perfusion. Using a 17 segment cardiac model (Figure 4-2), the average perfusion (as a percentage of the maximum perfusion) was calculated for each segment. The LVEF and the volume of the LV at systole and diastole were calculated from the reconstruction of the cardiac gated SPECT data. To minimize inter-operator variability in the calculated volumes and ejection fraction, the data was all processed by a single user. 


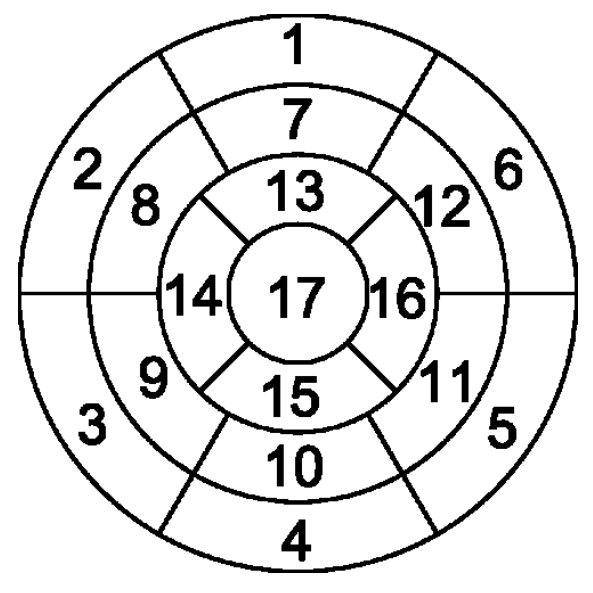

1. Basal anterior

2. Basal anteroseptal

3. Basal inferoseptal

4. Basal inferior

5. Basal inferolateral

6. Basal anterolateral

7. Mid anterior

8. Mid anteroseptal

9. Mid inferoseptal

10. Mid inferior

11. Mid inferolateral

12. Mid anterolateral

13. Apical anterior

14. Apical septal

15. Apical inferior

16. Apical lateral

17. Apex

Figure 4-2. Seventeen segment cardiac model. For analysis, myocardial perfusion is shown on a polar plot with the outermost ring corresponding to the basal section of the heart, the centre to the apex, and the angular segments divided up as shown.

To correct for changes in rat weight as the rats grew over the course of the study, the measured volumes were normalized based on the weight of the rat. Based on (Holt, Rhode, \& Kines, 1968) and (Dawson, 2001), which report a linear relationship between body weight and ventricular volume in mammals, we have scaled the LV volumes to an equivalent volume corresponding to the average weight of the rats in the group of interest. For the inter-scan repeatability we scale each volume by the ratio of the average weight of the rat over the five scans to the weight of the rat at the time of the scan. Likewise for the inter-rat repeatability, we correct the volumes to the average weight of all eight rats at that time point. Figure 4-3 shows the LV volumes measured for one of the rats before and after rescaling. 

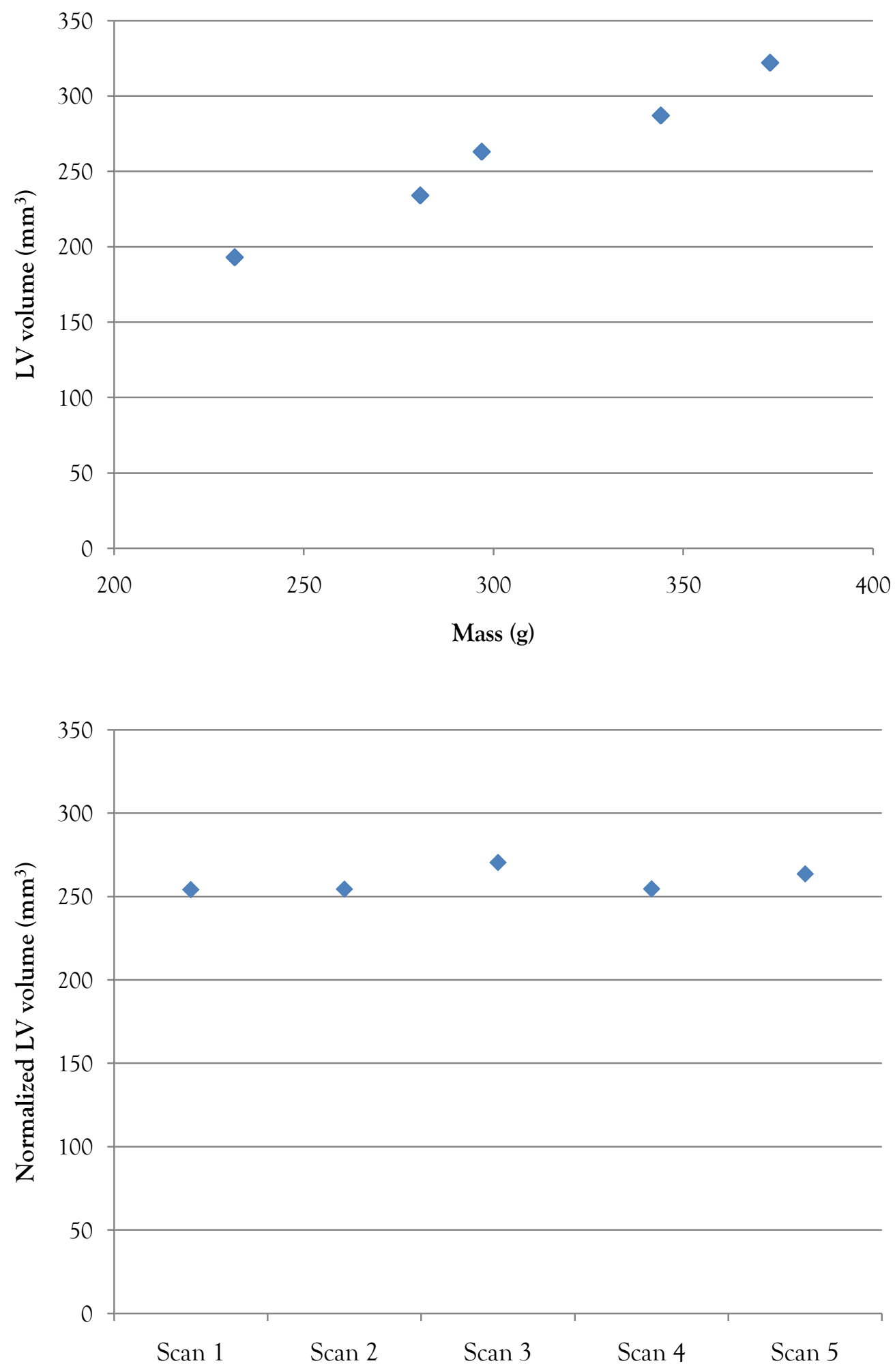

Figure 4-3. (top) Volume of the left ventricle of rat 1 plotted against the mass of the rat. (bottom) Volume of the left ventricle of rat 1 after normalizing the volumes based on the mass of the rat at the time of each scan. 


\subsubsection{Data analysis}

Both the normal and the MI studies comprised a total of eight rats, each scanned five times, for a total of forty sets of scan data per study. For the parameters calculated, intra-subject reproducibility was specified by the variation in an observed parameter when the same rat was scanned and analyzed repeatedly. Inter-rat reproducibility is specified by the variation in a parameter measured in a large group of rats. To calculate intra-subject reproducibility of a particular parameter, data were grouped by rat, the standard deviation of the parameter was calculated for each animal, and the standard deviations were averaged over all eight rats. Inter-rat reproducibility was calculated similarly, but with the data grouped by scan number, instead of by rat. The standard deviation of each parameter was calculated for each scan and then the standard deviations averaged over all five scans. The significance of the differences between reproducibility was assessed using Welch's t-test to compare the means of sample populations with unequal variances.

\subsubsection{Perfusion}

A normal database of myocardial perfusion was created by calculating the average and standard deviation of the perfusion of each segment of the myocardium across all scans. The data set used to create the normal database also contained the eight rats for which the functional data was analyzed and an additional four male Sprague-Dawley rats for which we had perfusion data, but no functional data, for a total of twelve rats (60 total scans).

The inter-scan and inter-rat reproducibilities were calculated for all seventeen segments. For the MI rats, we also calculated a z-score for each segment. The z-score was the difference between the measured perfusion in the segment and the corresponding segment in the normal database, divided by the standard deviation for that segment. We also calculated, as an overall measure of myocardial damage, a combined $z$-score for each scan, defined as the sum of the $z$-scores for all segments with a z-score less than or equal to -2.0 . We also measured the reproducibility of the perfusion 
measurements for the unaffected segments of the infarcted rat heart to evaluate the applicability of the normal rat database to infarcted rat models. We chose only those segments which had a normalized perfusion greater than $75 \%$ in all the rats scanned and compared the measured perfusion in those segments to the normal database.

\subsubsection{Functional measurements}

To factor out the normal growth of the rat from the inter-scan and inter-rat reproducibility of the volume measurements, the end diastolic (EDV) and end systolic (ESV) LV volumes, all volumes were normalized based on the weight of the rat as described above. For the inter-scan reproducibility calculations, the volumes were normalized to the average weight of each rat over the course of the study. For the inter-rat reproducibility, the volumes were normalized to the average weight of the rats in each scan. The reproducibility of the ejection fraction was also calculated, but in this case no normalization was required. The reproducibilities of the ESV, EDV, and ejection fraction were determined for the normal and the infarcted rats.

\subsection{Results}

\subsection{Scan}

At the time of the first scan, the average weight of the eight healthy rats was $245 \pm 15 \mathrm{~g}$. At the time of the final scan, the average weight of the rats was $383 \pm 41 \mathrm{~g}$. The eight infarcted rats began with weights of $432 \pm 59 \mathrm{~g}$, and had weights of $475 \pm 51 \mathrm{~g}$ by the final scan.

During acquisition, the healthy rats had an average heart rate of $320 \pm 30 \mathrm{bpm}$, and the respiration rate of $60 \pm 9$ breaths/minute. The infarcted rats had heart and respiration rates of $337 \pm 47 \mathrm{bpm}$ and $58 \pm 11$ breaths/minute, respectively. 
The total number of counts used to reconstruct the images was 7-12 million counts for the ungated images and about 1 million counts per phase for the gated images. On average the bad-beat rejection caused a loss of $15 \%$ of the counts in the gated data compared to the ungated data.

\subsubsection{Perfusion}

A normal database created from the scans of the normal rats is shown in Figure 4-4 and provides the average perfusion and standard deviation (over all scans) for each segment of the myocardium. Average segment perfusion ranges from $72.8 \%$ to $93.4 \%$ with standard deviations between $3.2 \%$ and $5.5 \%$. A region of comparatively low perfusion in the lateral wall near the apex was observed in all rats, and is reflected in the normal database. The reason for this is unclear. An apparent reduction in uptake could be caused by partial volume effects, but the myocardium does not appear thinner near the apex when dissected. The low perfusion region is however very reproducible across all of the rats we have scanned to date. 


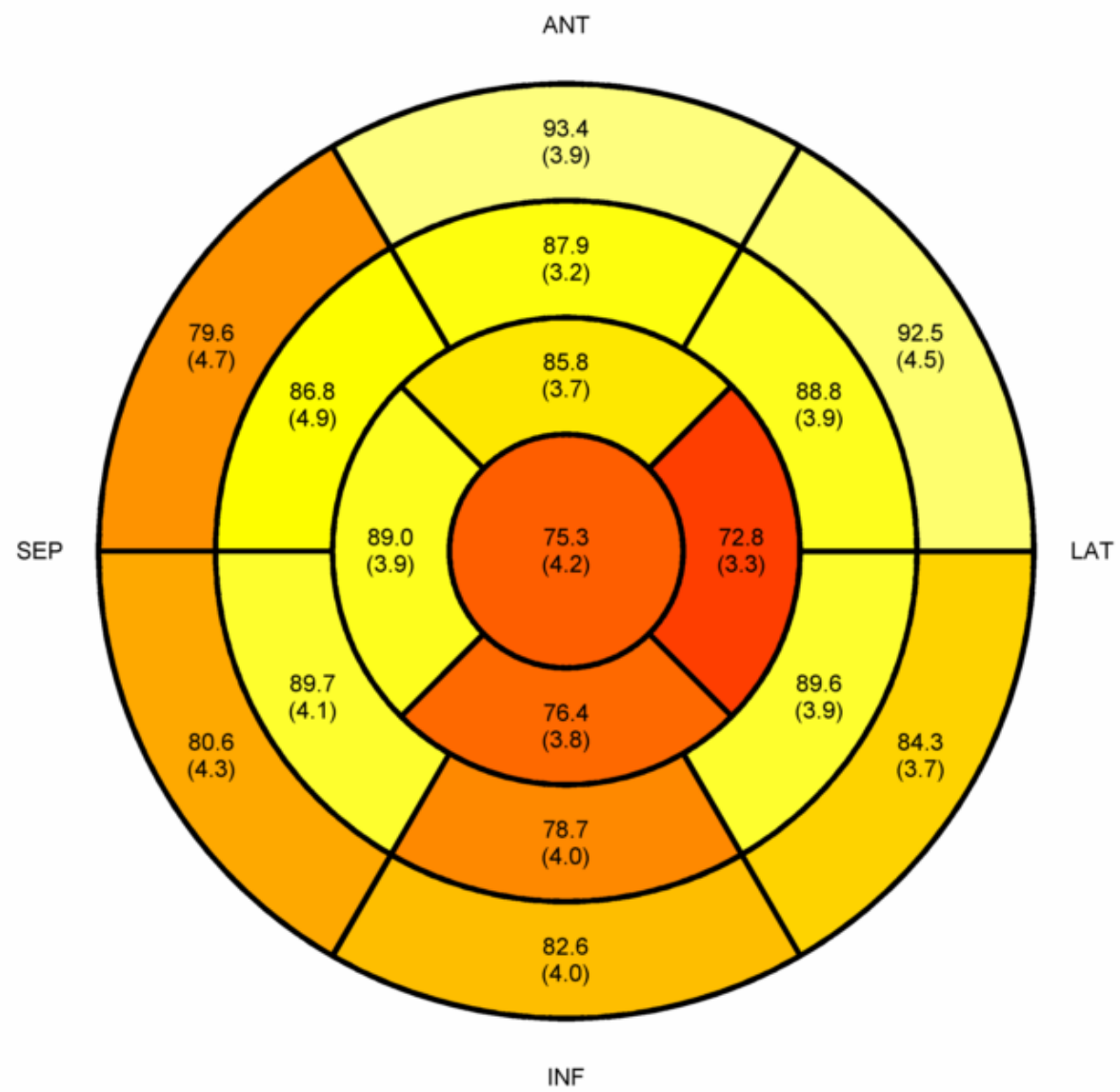

Figure 4-4. Normal database showing average relative perfusion and standard deviation of average perfusion for each segment of the myocardium.

The minimum, maximum, and average of the segment perfusion reproducibilities are listed in Table 4-1 for the normal rats and in Table 4-2 for the MI rats. The average inter-scan reproducibility of the segmental perfusion is $2.8 \pm 1.1 \%$, and the average inter-rat reproducibility is $4.0 \pm 0.8 \%$. The reproducibility of the segmental perfusion measurements is worse for the MI rats: $5.3 \pm 3.5 \%$ and $14.4 \pm 8.1 \%$ for the inter-scan and inter-rat reproducibility, respectively. 
Table 4-1. Reproducibility of cardiac perfusion measurements in normal rats

\begin{tabular}{lll}
\hline Inter-scan & Mean Perfusion & Reproducibility (segment) \\
\hline Least variable segment & $86.8 \%$ & $2.4 \pm 0.6 \%(8)$ \\
Most variable segment & $80.6 \%$ & $3.6 \pm 1.4 \%(3)$ \\
Average & $84.3 \%$ & $2.8 \pm 1.1 \%$ \\
& & \\
Inter-rat & Mean Perfusion & Reproducibility (segment) \\
\hline Least variable segment & $87.9 \%$ & $3.2 \pm 0.5 \%(7)$ \\
Most variable segment & $86.8 \%$ & $5.0 \pm 0.2 \%(8)$ \\
Average & $84.3 \%$ & $4.0 \pm 0.8 \%$ \\
\hline
\end{tabular}

Table 4-2. Reproducibility of cardiac perfusion measurements in myocardial infarct rats

\begin{tabular}{lll}
\hline \hline Inter-scan & Mean perfusion & Reproducibility (segment) \\
\hline Least variable segment & $21.4 \%$ & $3.1 \pm 2.2 \%(16)$ \\
Most variable segment & $76.5 \%$ & $7.1 \pm 5.0 \%(6)$ \\
Average & $63.1 \%$ & $5.3 \pm 3.5 \%$ \\
& & \\
Inter-rat & Mean perfusion & Reproducibility (segment) \\
\hline Least variable segment & $82.7 \%$ & $5.0 \pm 1.4 \%(8)$ \\
Most variable segment & $58.5 \%$ & $26.9 \pm 1.8 \%(11)$ \\
Average & $63.1 \%$ & $14.4 \pm 8.1 \%$ \\
\hline
\end{tabular}

If the segments are divided into uninfarcted segments (z-score $>-2.0)$, moderately infarcted (z-score between -2.0 and -4.0$)$, and severely infarcted $(z$-score $<-4.0)$, the average inter-scan segment perfusion reproducibilities were $3.6 \%, 5.2 \%$, and $7.2 \%$ respectively.

The validity of using the normal database for evaluation of MI rats was assessed by comparing only those segments in the MI scans with an average normalized perfusion exceeding $75 \%$ in all eight rats to the normal database. In the rats studied, three segments, all in the septal wall of the heart, met this criterion. These segments had average perfusions of $86.0 \pm 6.6 \%, 82.7 \pm 5.3 \%$, and $86.5 \pm$ $5.4 \%$. The corresponding segments in the normal database were $89.7 \pm 4.1 \%, 86.8 \pm 4.9 \%$, and $89.0 \pm 3.9 \%$. 


\subsubsection{Reproducibility of infarct severity measurements}

Figure 4-5 shows the z-scores for one of the rats. A combined z-score indicating the overall size and severity of the infarct was calculated for each of the rats, listed in Table 4-3. The average score for each rat ranged from -54 for the least severely infarcted animal to -143 for the most severely infarcted. For individual rats, the average inter-scan standard deviation of the combined $z$-score was 8. The average standard deviation of the inter-rat combined z-scores was 28 .
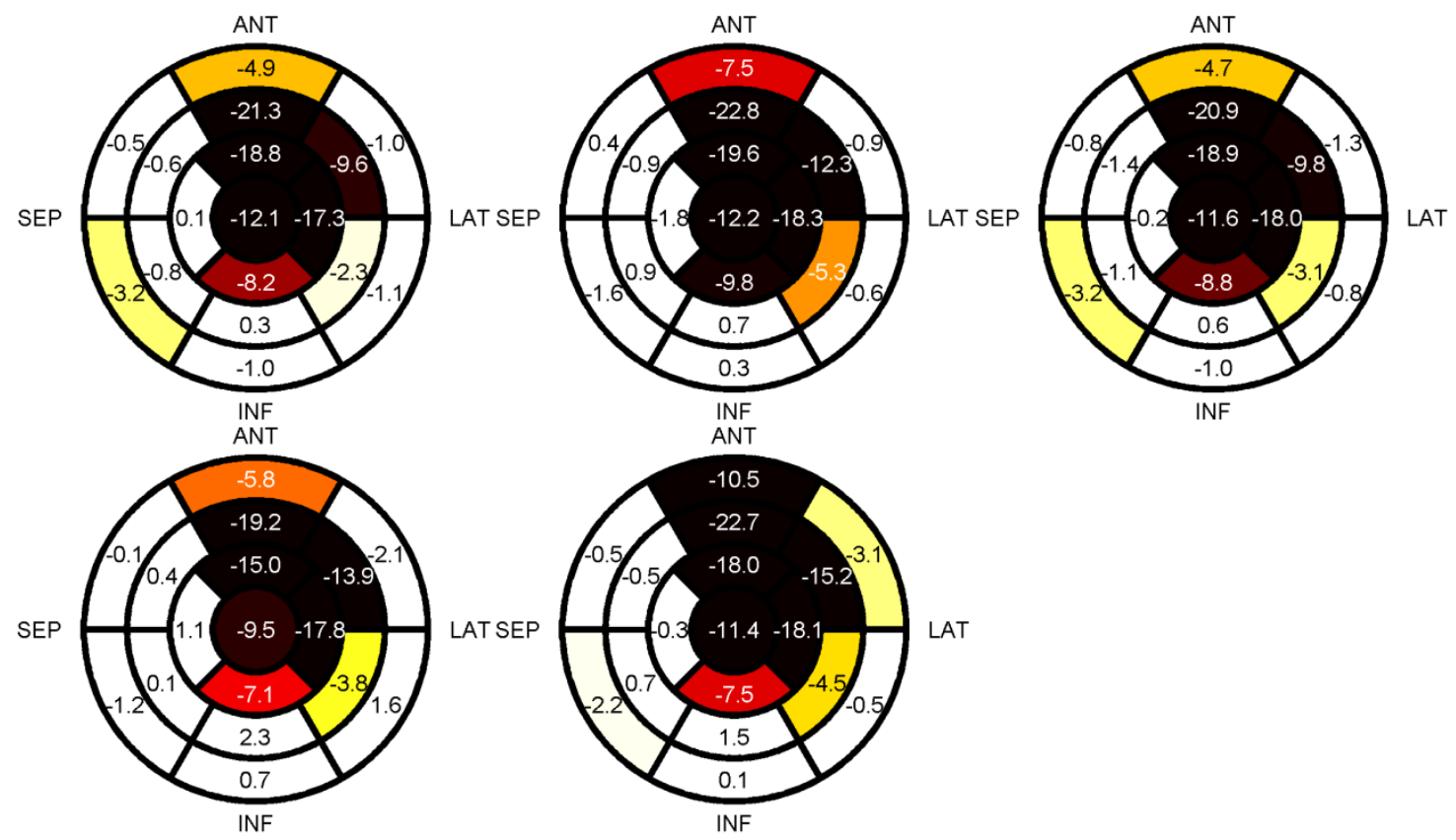

Figure 4-5. Z-scores for all five scans of the one of the infarcted rats.

Table 4-3. Average combined z-scores for five scans of eight infarcted rats.

\begin{tabular}{ll}
\hline Rat & Combined $z$-score \\
\hline 1 & $-88 \pm 22$ \\
\hline 2 & $-115 \pm 7$ \\
\hline 3 & $-102 \pm 8$ \\
\hline 4 & $-84 \pm 8$ \\
\hline 5 & $-54 \pm 5$ \\
\hline 6 & $-143 \pm 5$ \\
\hline 7 & $-84 \pm 8$ \\
\hline 8 & $-82 \pm 4$ \\
\hline
\end{tabular}




\subsubsection{Functional measurements}

\subsubsection{Reproducibility of systolic and diastolic volumes}

The mean values and the reproducibilities of the systolic and diastolic LV volumes for the normal and MI rats are shown in Table 4-4 and Table 4-5, respectively. For both, the inter-rat variation is significantly larger than the inter-scan variation (ESV: $p=0.003$, EDV: $p=0.006$ ). Inter-scan reproducibility is $4-7 \%$ of the total volume and inter-rat reproducibility is $9-12 \%$ of total ventricle volume for the normal rats. In the myocardial infarct rats, the inter-rat variations of the systolic and diastolic volumes are much larger than inter-scan variances ( $\mathrm{p}<0.001$ in both cases).

For the myocardial infarct rats, the inter-scan variances are larger in absolute terms than that for normal rats, but the average volume is also larger because the MI rats were older and because of myocardial remodelling following the infarction. Expressed as a percentage of average ventricular volume, the inter-scan reproducibility is $6-8 \%$, marginally worse than the relative inter-scan reproducibility for the normal rats.

Inter-rat reproducibility is much worse for the myocardial infarct rats: $15-26 \%$ of the average volume of the LV.

\subsubsection{Reproducibility of ejection fraction}

Ejection fraction reproducibility for the normal rats is also included in Table 4-4 and Table 4-5. Unlike for the LV volumes, no significant difference is seen between the reproducibilities of $3.1 \%$ and $3.3 \%$ for the inter-rat and inter-scan ejection fraction measurements respectively $(p=0.86)$. The inter-scan ejection fraction reproducibility for the myocardial infarction rats is not significantly different than the reproducibility of the normal rats at 3.6\% $(\mathrm{p}=0.64)$. Inter-rat reproducibility of $8.1 \%$ is significantly worse than inter-scan reproducibility for the myocardial infarction rats $(p=0.01)$. 
Table 4-4. Reproducibility of functional measurements in normal rats.

\begin{tabular}{lll}
\hline \hline Inter-scan $(\mathbf{n}=8)$ & Mean & Reproducibility \\
\hline Ejection Fraction & $57 \%$ & $3.1 \pm 1.6 \%$ \\
Diastolic Volume* & $470 \mathrm{~mm}^{3}$ & $20 \pm 7 \mathrm{~mm}^{3}$ \\
Systolic Volume* & $203 \mathrm{~mm}^{3}$ & $13 \pm 6 \mathrm{~mm}^{3}$ \\
& & \\
Inter-rat (n=5) & Mean & Reproducibility \\
\hline Ejection Fraction & $57 \%$ & $3.3 \pm 1.5 \%$ \\
Diastolic Volume* & $470 \mathrm{~mm}^{3}$ & $44 \pm 16 \mathrm{~mm}^{3}$ \\
Systolic Volume* & $204 \mathrm{~mm}^{3}$ & $24 \pm 5 \mathrm{~mm}^{3}$ \\
\hline
\end{tabular}

*Volumes are post-normalization. Since the inter-scan data is normalized to the average weight of each rat, and the inter-rat data is normalized to the average weight of all rats for each scan, the means differ slightly.

Table 4-5. Reproducibility of functional measurements in myocardial infarct rats.

\begin{tabular}{lll}
\hline \hline Inter-scan $(\mathbf{n}=8)$ & Mean & Reproducibility \\
\hline Ejection Fraction & $45 \%$ & $3.6 \pm 2.4 \%$ \\
Diastolic Volume* & $834 \mathrm{~mm}^{3}$ & $49 \pm 12 \mathrm{~mm}^{3}$ \\
Systolic Volume* & $462 \mathrm{~mm}^{3}$ & $37 \pm 17 \mathrm{~mm}^{3}$ \\
& & \\
Inter-rat (n=5) & Mean & Reproducibility \\
\hline Ejection Fraction & $45 \%$ & $8.1 \pm 2.4 \%$ \\
Diastolic Volume* & $832 \mathrm{~mm}^{3}$ & $126 \pm 20 \mathrm{~mm}^{3}$ \\
Systolic Volume* & $462 \mathrm{~mm}^{3}$ & $121 \pm 14 \mathrm{~mm}^{3}$ \\
\hline
\end{tabular}

*Volumes are post-normalization. Since the inter-scan data is normalized to the average weight of each rat, and the inter-rat data is normalized to the average weight of all rats for each scan, the means differ slightly.

\subsection{Discussion}

In every case, reproducibility, as measured by the standard deviation of repeated measurements, was worse for inter-rat measurements than for inter-scan measurements, underscoring the benefit of using non-invasive methods like SPECT for longitudinal studies. The benefits of inter-scan over inter-rat comparison are even more evident in rats with an induced myocardial infarction, where the underlying physiological variance between subjects is much greater than in normal rats. This reflects the difficulty of creating a consistent infarction across multiple animals using surgical methods. It is most evident in the large inter-rat variation in z-scores and ejection fraction in the 
myocardial infarct group compared to the inter-scan variation of the same parameters. From the scans it was visibly evident that the region of infarcted tissue was much larger in some rats than in others. Severely infarcted myocardia are also more difficult to align consistently during analysis, where the viable tissue remaining may not provide a clear image of the rotation and extent of the heart. The greater uncertainty in the position of the severely infarcted segments may contribute to the poorer reproducibility of the perfusions measurements in the more severely infarcted segments. For the MI rats, the inter-scan reproducibility of the segment perfusion decreases as the severity of the perfusion defect increases. Inter-scan reproducibility of the uninfarcted segments is no worse than in normal rats. Furthermore, measurements of perfusion in uninfarcted segments of the MI rats were consistent with the same measurements made in normal rats, summarized in the normal database. In severely infarcted segments, the inter-scan standard deviation of the relative perfusion was about twice as large as in the uninfarcted segments.

At this time, data on the reproducibility of cardiac SPECT studies in small animals are very limited. Moreover, direct comparison between our work and that of Vanhove et al., who reported reproducibility in terms of the average pairwise differences between LV volumes measured one week apart, is difficult. We note that the magnitudes of the pairwise differences observed by Vanhove et al. are consistent with variability observed in our study. Comparison with similar studies in human subjects is also challenging, as few studies have investigated the repeatability of measurements over repeated studies in humans. (Xu, et al., 2010) evaluated the reproducibility of functional measurements in humans and report a $95 \%$ confidence interval for the test-retest reproducibility of ejection fraction measurement in humans of $(-10.1 \%, 10.2 \%)$. The inter-scan reproducibility of ejection fraction observed in rats of $3.3 \%$ equates to an expected $95 \%$ CI for repeated measurements of $\pm 9 \%$, consistent with the results of the human study. 
Some of the variation in the measured values of segment perfusion, ejection fraction, and LV volume reflect actual differences in the physiology; others arise from inherent uncertainties in the procedure. Over time, gradual changes in the physiology are expected. Despite the effort made to maintain a consistent physiological state for each scan, some confounding factors, such as the level of anaesthetic, are unavoidable. Minor differences in physiological state are reflected in the ranges of heart and breathing rates between different scans and different animals. Inter-rat variations were minimized by using animals of the same age, sex, and strain, but differences between individual animals still exist and contribute to the variations observed in the measured parameters. In clinical imaging, attenuation causes sex-specific artefacts and requires the use of gender-specific databases. With rats, the impact of attenuation is greatly reduced due to the small size of the subject and we expect the reproducibility to be very similar for female rats of the same strain. Nevertheless, these results are specific to male rats and the effect of gender was not evaluated.

Variations in the scanning and analysis process unrelated to variations between rats also influence the repeatability. Variations would include exact placement of the animal, and noise in the imaging process. Image analysis introduces additional operator-dependant factors in the manual rotation and alignment of the reconstructed image and the placement of the valve plane. In this study, all image processing was performed by a single operator to minimize inter operator variation. The use of multiple operators for data analysis would increase the measured uncertainties. However, the inter-observer variability in small-animal myocardial perfusion SPECT reported by Vanhove is comparatively small and not significantly different from the intra-observer variability.

Increasing the number of frames used for ECG gating may improve the absolute accuracy of the LV volume measurements, particularly at systole, and the accuracy of the ejection fraction measurement. However, without increasing the scan duration, the additional subdivision of the 
data into a greater number of time divisions would increase the noise in each reconstructed frame, potentially degrading the accuracy and reproducibility, though to what degree is uncertain.

One of the major advantages of using SPECT to evaluate changes in the living subject is the ability to use an animal as its own control. We have demonstrated that the inter-scan reproducibility of LV volume measurements is significantly better than the inter-rat reproducibility. Relative to studies which rely entirely on inter-rat comparison, as when subjects must be sacrificed for analysis, SPECT permits a significant increase in statistical power with the same sample size, or equivalent statistical power with smaller sample size. For example, consider a study of ejection fraction in infarcted rats wherein we wanted to detect a change of $5 \%$ with an $\alpha$ of 0.05 and a power of 0.95 . Using two independent groups of rats, each with a standard deviation of ejection fraction of $8.1 \%$ when measured using microSPECT, a total sample size of 140 rats would be required. If the study were done as a serial study with each animal compared against itself (before and after treatment), the standard deviation of the difference is reduced to $5.1 \%$ and a paired t-test requires only 16 animals, a nine-fold reduction.

The linear model we used to correct for normal growth exhibited reasonable conformity to the growth trends observed in the healthy rats we studied which had weights ranging from 200-450 g. The disease state of the animal model may limit the applicability of this linear correction. Although we also used the same assumed weight-LV volume relationship to normalize the data in the MI rats included in this study, the physiological consequences of an induced myocardial infarction may change the relationship between the normal heart volume and the weight of the rat, undermining the validity of this assumption. Any error in this assumption was mitigated, however, by the shorter duration of the MI study. Because the MI rats were scanned over a shorter period of time, they 
grew comparatively less during the study and consequently required much less normalization than did the normal rats.

To measure the perfusion, LV volumes, and ejection fraction we used 4D-MSPECT (Invia, Ann Arbor, MI). Though 4D-MSPECT has been validated for human cardiac measurements (Nakajima, Higuchi, Taki, Kawano, \& Tonami, 2001), certain assumptions about the shape and thickness of a human myocardium may not be entirely appropriate for a rat heart. In particular, 4D-MSPECT will not process a rat-sized heart. As noted in the methods section, we scaled the apparent voxel size in the reconstructed heart data by a factor of 10 . The LV volume calculated by the software changed substantially with changes to the apparent voxel size. Within the range of 8-11 for the scaling factor, the volumes were constant and consistent with measurements of normal rat EDV by others (Vanhove, Lahoutte, Defrise, Bossuyt, \& Franken, 2005), (Wise, Huang, Gresham, Al-Shafei, Carpenter, \& Hall, 1998), (Nahrendorf, et al., 2001), (Croteau, et al., 2003). This method was further validated using a rat myocardium phantom, scanned with the same protocol as the rats in the study. The volume calculated by 4DM-SPECT was approximately $20 \%$ larger than the true volume, which we believe to be a consequence of the software 'rounding off' the flat bottom of the phantom to make it more heart-like, increasing the apparent volume. These uncertainties in the absolute accuracy of the volume measurement are not expected to have a significant impact on the conclusions of this paper.

\subsection{Conclusion}

We have created a normal database for myocardial perfusion of male Sprague-Dawley rats imaged with ${ }^{99 \mathrm{~m}} \mathrm{Tc}$-tetofosmin. We have also measured the repeatability of perfusion measurements made in normal rats and in rats with a coronary artery ligation induced myocardial infarction. Finally, we 
have measured the reproducibility of NanoSPECT functional measurements of LV diastolic volume, systolic volume and ejection fraction. 


\section{Attenuation and Scatter Correction of pre-clinical SPECT Data}

The various methods applied for attenuation and scatter correction have already been covered in Chapter 2 (\$2.3-2.4), and the details of implementation covered in Chapter 3 of this work. In this chapter we describe the calibration of the nanoSPECT scanner to obtain quantitatively measurements of tracer concentration in phantoms, then apply this quantitative method, with the attenuation and scatter correction to phantoms containing known concentrations of activity, and finally attempt to measure, quantitatively, the activity uptake, in vivo, in rat myocardia.

\section{I Materials and method}

\section{I.I CT scans}

CT scans were performed with the cone-beam CT scanner of the nanoSPECT SPECT/CT scanner (Bioscan, Washington, DC). A beam energy of $45 \mathrm{kV}$ was used for optimal soft tissue contrast and all scans in this study followed a circular trajectory with 360 equally spaced projections. Because beam hardening artefacts are evident even in small phantoms, all projections were corrected for beam hardening prior to reconstruction using a polynomial curve fitted to the attenuation of various thicknesses of water. The corrected projections were reconstructed with the FDK algorithm 
for cone-beam CT at a resolution of $0.2 \mathrm{~mm}$ on a $384 \times 384 \times 384$ grid. The reconstructed CT image was then downsampled to $128 \times 128 \times 128$ at a resolution of $0.6 \mathrm{~mm}$ and converted to a linear attenuation coefficient map for each voxel following a bilinear rescaling of the CT number.

\subsubsection{SPECT scans}

All SPECT scans were performed on the nanoSPECT/CT system with multiplexing multi-pinhole apertures. Each aperture plate consisted of 9 pinholes each with an opening diameter of $2.5 \mathrm{~mm}$ (APT2 apertures). A total of 48 projections over $360^{\circ}$ were acquired following a helical scan with a total bed travel of $25 \mathrm{~mm}$ and a scan duration of $150 \mathrm{~s}$ per frame for a total scan duration of 30 min. At each angle, projections were acquired for two energy windows: a primary energy window from 126-154 keV, and a downscatter energy window from 97-125 keV. SPECT data were reconstructed with the software described in $\$ 3.3$.

Two methods were tested for scatter correction. The first method was to use a reduced attenuation coefficient for reconstruction by scaling the narrow beam linear attenuation map by a factor of 0.83 $\left(\mu_{\mathrm{H} 2 \mathrm{O}}=0.12 \mathrm{~cm}^{-1}\right)$ to approximate attenuation in a broad-beam geometry $(\$ 2.4 .1 .1)$. The second method was dual energy window scatter correction (\$2.4.1.3) and was implemented by Gaussian smoothing the downscatter window projections $(\mathrm{FHWM}=3.0 \mathrm{~mm})$, scaling them by a constant scatter coefficient, $\mathrm{k}$, and adding the estimated scatter distribution to the forward projected estimate for each iteration of the reconstruction.

The dual-energy window correction method approximates the scatter contribution to the projection data by assuming a fixed ratio $(\mathrm{k})$ between the number of scattered photons in the photopeak widow and the number of photons detected in the down-scatter window. While this is a reasonable approximation, in practice the ratio depends on multiple factors including the scanner and source geometry and is not genuinely spatially constant. Determining the appropriate scatter correction 
coefficient to use for quantitative reconstruction is a heuristic process. In this study, the optimal kvalue was experimentally determined following the approach of (Koral, Swailem, Buchbinder, Clinthorne, Rogers, \& Tsui, 1990).

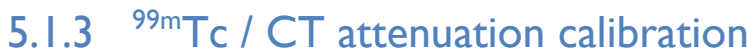

A set of tissue-equivalent cylindrical phantoms with relative electron densities from $\rho_{e}^{w}=0.28$ (lung) to $\rho_{e}^{w}=1.69$ (cortical bone) was scanned to measure the CT number corresponding to each material. A CT scan of each phantom was acquired at $45 \mathrm{kV}, 1.5 \mathrm{~s} /$ projection, and 360 projections, and the average CT number calculated for each phantom.

The conversion from CT number to $140 \mathrm{keV}$ photon linear attenuation coefficients assumes a bilinear relationship between the two (see (Blankespoor, et al., 1996) for example). The photon attenuation of water and cortical bone were based on NIST tables of mass attenuation coefficients (Hubbell \& Seltzer, 2004). For CT densities below that of water, the $140 \mathrm{keV}$ photon attenuation was estimated as follows:

$$
\mu\left(\mathrm{cm}^{-1}\right)=0.15 \frac{\mathrm{HU}}{\mathrm{HU}_{\text {water }}}
$$

For CT densities above that of water, the $140 \mathrm{keV}$ photon attenuation was estimated by:

$$
\mu\left(\mathrm{cm}^{-1}\right)=0.15+0.11 \frac{\mathrm{HU}-\mathrm{HU}_{\text {water }}}{\mathrm{HU}_{\text {cortical bone }}-\mathrm{HU}_{\text {water }}}
$$

To measure the broad-beam attenuation of $140 \mathrm{keV}$ photons, a point source was placed in the scanner at a distance of approximately $35 \mathrm{~cm}$ from the scintillator surface. The count rate was measured both with and without the phantom placed over the point source. From the relative count rates and the length of the phantom $(70 \mathrm{~mm})$, a broad-beam linear attenuation coefficient for $140.5 \mathrm{keV}$ photons was calculated for each phantom. The average ratio of the broad-beam to narrow-beam attenuation coefficients was measured to be 0.83 . 


\section{I.4 Preparation of activity dilutions}

${ }^{99 \mathrm{~m}} \mathrm{Tc}$ for all phantom experiments was supplied in the form of sodium pertechnetate $\left(\mathrm{NaTcO}_{4}\right)$. For most of the calibration and phantom experiments, the activity was diluted to obtain a source with a precisely known activity concentration.

The activity concentration was prepared by diluting the source activity with water in a $25.0 \mathrm{~mL}$ $( \pm 0.1 \%)$ volumetric flask. The activity concentration $c_{i}$ was calculated as:

$$
c_{i}=\frac{A_{\text {initial }}-A_{\text {residual }}}{25.0 \mathrm{~mL}}
$$

Here, $A_{\text {initial }}$ and $A_{\text {residual }}$ are the decay corrected initial and residual activities of the syringe in which the sodium pertechnetate is supplied. This approach to calculating the activity concentration was validated by measuring the activity of a small volume of the dilute activity added in $10 \mu \mathrm{L}$ increments to a small vial in the dose calibrator and measuring the slope of the resulting plot.

\section{I.5 Quantitative calibration}

Calibration to allow the raw 'counts' of a reconstructed SPECT image to be converted to a quantitative activity concentration (e.g. $\mathrm{kBq} / \mathrm{mL}$ ) was done using small sources containing carefully measured activity.

Eight point sources were created by placing drops of ${ }^{99 \mathrm{~m}} \mathrm{Tc}$ in one end of a glass capillary tube, sealed at the ends with putty. The activities of the point sources measured by the dose calibrator ranged between 1.2-8.8 MBq $(33-240 \mu \mathrm{Ci})$. The point sources were scanned and reconstructed following the same protocol used for the phantoms in §5.1.1 and §5.1.2.

To obtain a calibration factor from a particular point source, the activity, $A$, was measured in the dose calibrator (Capintec CRC-25R), and corrected to obtain the activity at the start of the SPECT 
scan. A region of interest was drawn around the reconstructed point source and the total counts, $N$, within the ROI was measured.

The calibration factor was then determined as follows:

$$
k_{a}=\frac{A}{N / t}
$$

where $t$ is the acquisition duration for a single projection.

Unfortunately, the ratio of reconstructed counts to measured activity exhibited significant variation from one point source to another; the calibration resulting from different point sources differed by up to $13 \%$, and the standard deviation was $4 \%$ of the mean value.

Similar results were obtained when creating an additional eight point sources using a micropipette to prepare $10 \mu \mathrm{L}$ samples from diluted activity with accurately measured activity concentrations on the order of $5-7 \mathrm{kBq} / \mu \mathrm{L}$. The diluted activity was prepared by measuring the activity of a small volume of $\mathrm{NaTcO}_{4}$ (approximately $5 \mathrm{mCi}$ ) in a dose calibrator and diluting it with water to a final volume of $25.0 \mathrm{~mL}$. These calibration sources had activities of $52-60 \mathrm{kBq}(1.4-1.6 \mu \mathrm{Ci})$. The calibration factor was calculated as:

$$
k_{a}=\frac{c_{i} V}{N / t}
$$

where $c_{i}$ is the activity concentration, $V$ is the volume of the point source $(10 \mu \mathrm{L})$, and $t$ is the acquisition duration for a single projection.

Again, the standard deviation of the activity to reconstructed counts ratio was $4 \%$ of the mean ratio, and the difference between the max and min ratio was $12 \%$ of the mean value. 
The same variation between point sources was observed when reconstructing the point sources using the vendor reconstruction software instead of our own.

The source of the large degree of uncertainty in the calibration factor remains unclear. The variability may point to a fine grained variation in the sensitivity of the scanner at the sub-voxel level of resolution, or unidentified sources of measurement error as the uncertainty in the obtained calibration factor exceeds that of the activity and volume measurements of the calibration source.

With slightly larger calibration sources, the results of the calibration were much more reproducible. When a $150 \mu \mathrm{L}$ volume of the diluted activity was imaged in the SPECT scanner, reconstructed, and used to derive the calibration coefficient using Equation (5-5), the results varied by less than $1 \%$.

\subsubsection{Phantom experiment}

Five $2.5 \mathrm{~mL}$ vials (Figure 5-1) were filled with the diluted activity solution prepared as in the previous section. Four of the vials were then mounted at the centre of water-filled polyethylene bottles with inside diameters of $23,37,49$, and $52 \mathrm{~mm}$. The fifth was scanned with no additional attenuating and scattering media. All phantoms were scanned in the CT scanner at a beam energy of $45 \mathrm{kVp}$ and then in the micro SPECT scanner with the APT2 apertures. The data acquisition was repeated a total of 4 times on different days with new activity sources. Uncertainty was estimated as the standard deviation in the results over the 4 scans.

The reconstructed images were analyzed using InVivoScope software (Bioscan, Washington, DC). The volume of interest (VOI) had a length of $15 \mathrm{~mm}(25$ slices $\times 0.6 \mathrm{~mm} / \mathrm{slice})$ and enclosed the entire active volume in the trans-axial plane. The axial position of VOI was fixed to be well within the active volume of the cylinder to avoid partial volume effects. The vials containing activity had an internal diameter of $8.7 \pm 0.1 \mathrm{~mm}$, for a total VOI volume of $896 \pm 23 \mu \mathrm{L}$. 


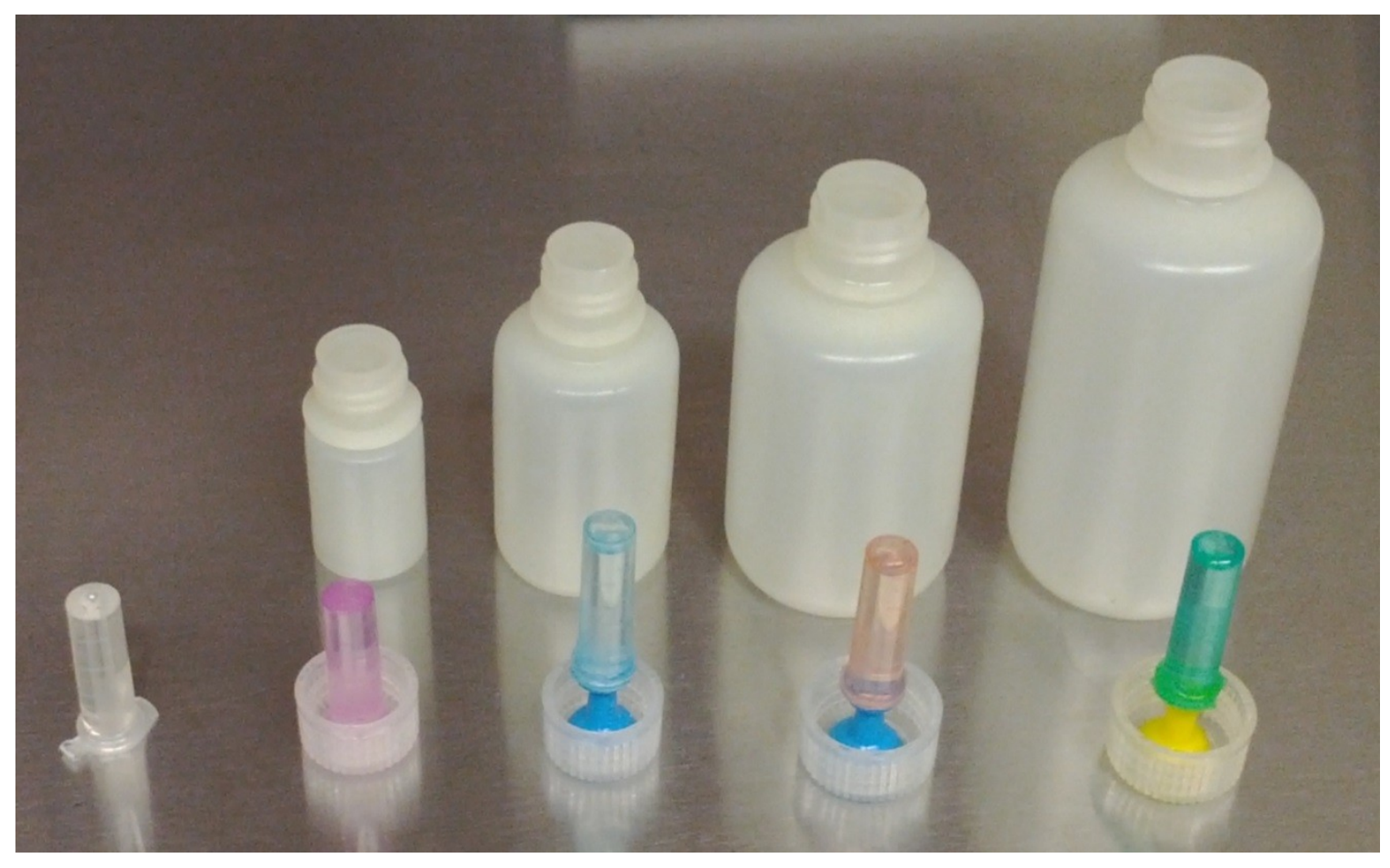

Figure 5-1. Phantoms used for evaluation of absolute activity quantification. Small vials (front row) were filled with activity and placed inside the larger bottles.

\section{I.7 Heart-liver-lung phantom}

For the phantom study, activity was confined to the region of interest, i.e. background activity was zero. In the case of cardiac imaging, the lungs, which wrap around the posterior and lateral aspect of the heart, typically contain a low level of activity. The liver, directly inferior to the apex of the heart, can also take up a significant amount of the injected tracer. To investigate the influence of activity from organs adjacent to the heart on the activity measured in the heart, and on how activity from adjacent sources may influence the scatter correction algorithms, more complex phantom studies were also done with simulated lung and liver activity. To investigate the liver contribution, a vial of activity ('heart') was placed within a wet cylindrical sponge ('lung') and a larger bottle containing activity was placed adjacent ('liver') (Figure 5-2). Scans were performed with and without activity in the 'liver'. 


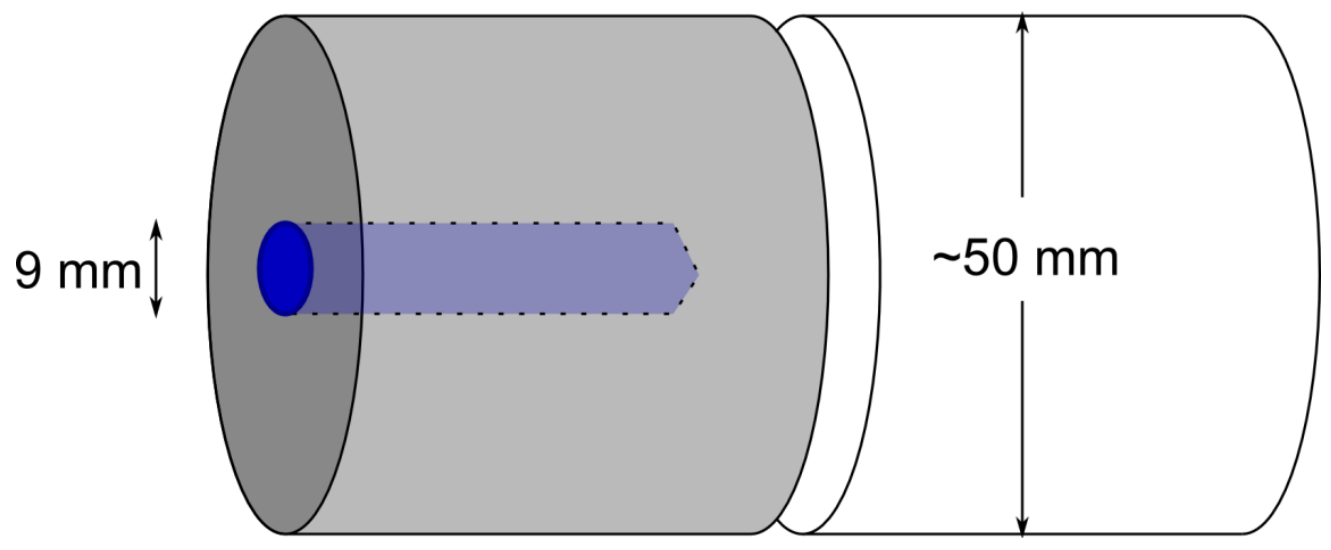

Figure 5-2. Heart/Lung/Liver phantom. The "heart" (blue) was a vial filled with a known concentration of activity. The "lungs" (grey) were made of sponge moistened with water, with or without activity. The "liver" (white) was filled with activity for some experiments.

We also simulated the effect of background activity in the lungs on the quantitative accuracy of the cardiac activity measurement using the same phantom but with the sponge moistened with radioactive water, simulating the low level of activity in the thorax surrounding the heart in rat scans. The activity concentration of the water used to moisten the sponge for the hot phantom was approximately half of the activity concentration within the vial. In the sponge, the background activity was approximately $17 \%$ of the activity in the vial. (This is slightly higher than the ratio of background to myocardium measured for one of the rats, where the average activity concentration of the lungs and thorax was approximately $11 \%$ of that in the myocardium.)

CT scans before and after the SPECT scans were used to confirm that there was no appreciable settling of the water within the moist sponge during the SPECT scan of the 'lung' phantom.

\section{I.8 In vivo}

Five Sprague-Dawley rats (age: 15 wks, weight: $413 \pm 12 \mathrm{~g}$ ) were injected with Tc-99m tetrofosmin, anaesthetized with $2-3 \%$ isoflorane, and scanned 30 minutes post-injection. Both a $45 \mathrm{kVp}$ conebeam CT scan and a 30 minute SPECT scan were acquired. The rats were sacrificed and the heart 
activity was measured in a well counter. The SPECT scans were reconstructed with and without attenuation and scatter correction. The number of counts contained within a VOI encompassing the heart was converted to absolute activity for comparison with the activity measured in the well chamber.

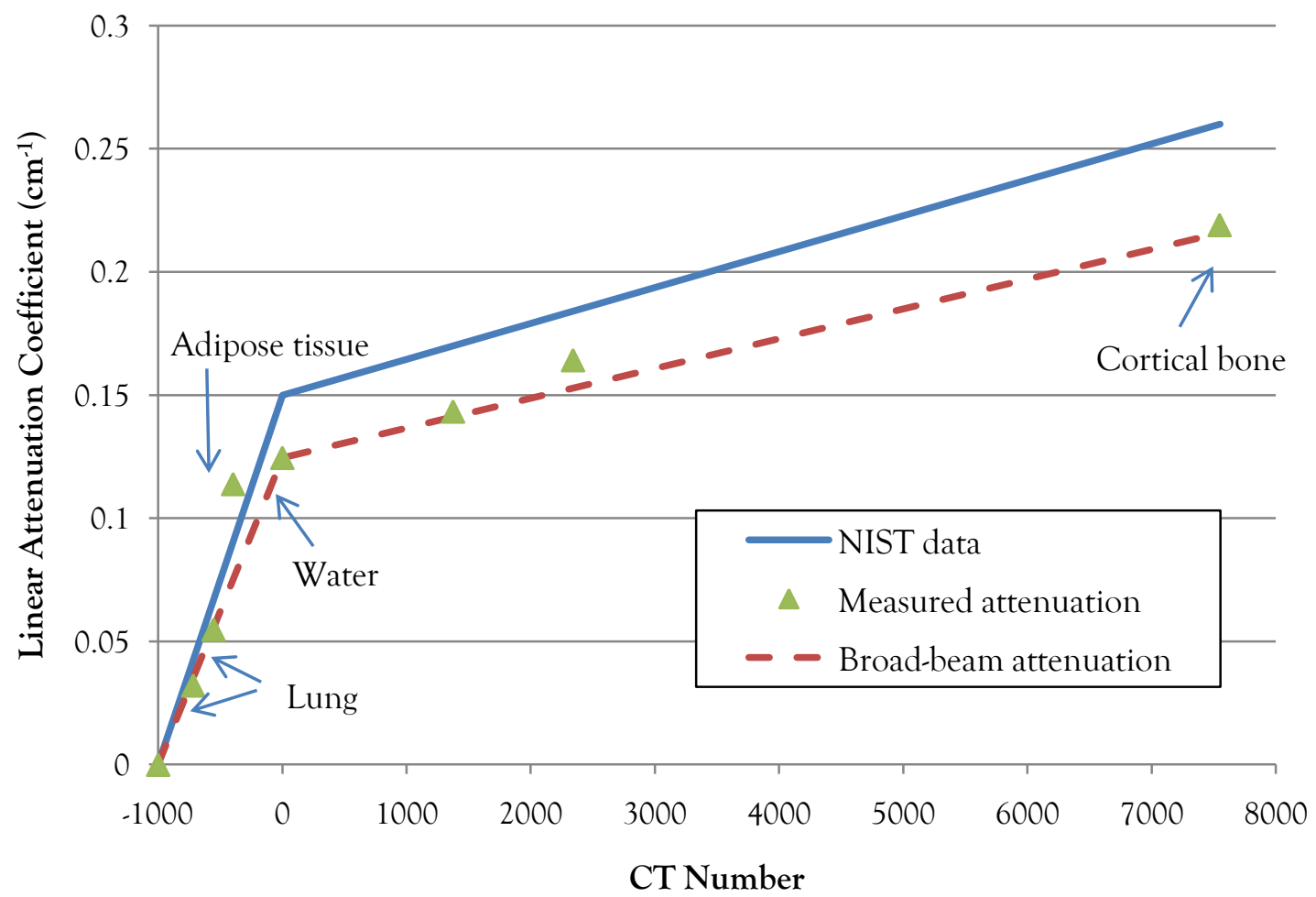

Figure 5-3. Conversion from CT number (for $45 \mathrm{kVp} \mathrm{X}$-ray) to linear attenuation coefficients for $140 \mathrm{keV}$ photons. Linear attenuation coefficients (LAC) for attenuation correction (solid line) are based off of NIST values. The dashed lines shows the conversion used for broad-beam scatter correction, obtained by scaling the LAC by a factor of 0.83 . The measured CT number and broad-beam LAC are also shown for several tissue equivalent phantom materials.

\section{I Results}

\section{I.I CT/SPECT attenuation calibration}

Figure 5-3 shows the measured CT density for various tissue-equivalent phantoms, and shows the attenuation coefficient of $140 \mathrm{keV}$ photons based on NIST data and the measured board-beam attenuation for the same material. 
Measured broad-beam attenuation of the $140 \mathrm{keV}$ photons by the tissue equivalent phantoms was between $75 \%$ and $89 \%$ of the NIST-derived attenuation coefficients, and averaged $83 \%$ of the latter. Based on these measurements, a scaling factor of 0.83 was applied to the linear attenuation coefficient to calculate the attenuation maps for broad-beam scatter correction.

\section{I.2 Convergence of OS-EM reconstruction}

Figure 5-4 shows the total number of counts within a volume of interest enclosing an $18 \mathrm{~mm}$ length of the vial in the largest phantom over the full set of iterations. The total number of counts quickly settles within two iterations and as the number of subsets decreases, the variation in the number of counts in the ROI from one subset to the next decreases as well, from a standard deviation of $0.6 \%$ with 6 subsets, to less than $0.01 \%$ with only a single subset (equivalent to ML-EM reconstruction). A 3\% increase in the calculated activity in the $\mathrm{VOI}$ is observed between iterations using a subset of the full projection data (OS-EM) and those using all of the projections (ML-EM). Since the ML-EM algorithm converges to the maximum-likelihood solution for the full dataset, and the OS-EM algorithm is likely to cycle between the maximum likelihood solutions of each subset, the most reproducible quantitative calibration results from full ML-EM reconstruction, at least for the final few iterations. The cycling is evident in the periodicity of the total counts within an ROI, shown in Figure 5-4; during the OS-EM portion of the reconstruction the total counts cycles with a period equivalent to the number of subsets. For the final eight updates, where all projections are included, the number of counts is stable. Inclusion of attenuation and scatter correction does not affect the rate of convergence of the reconstruction. 

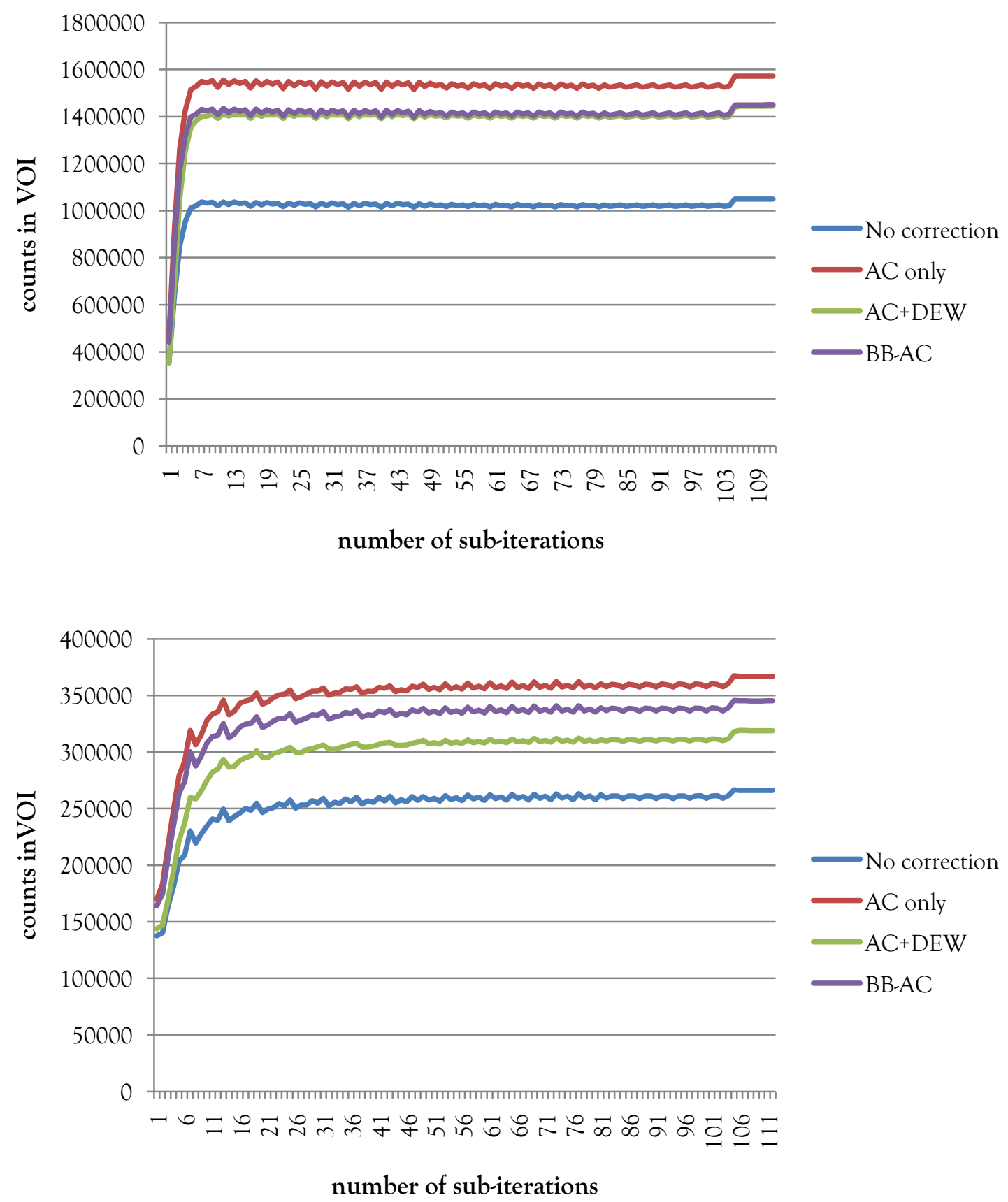

Figure 5-4. Total number of counts within a volume of interest for each sub-iteration of the OSEM reconstruction of a phantom (top) and a rat injected with ${ }^{99 \mathrm{~m}}$ Tc-tetrofosmin (bottom). Sub-iterations $1-48,6$ subsets; sub-iterations 49-80, 4 subsets; sub-iterations 81-104, 3 subsets. Final 8 iterations (105-112), use all projections.

Figure 5-4 also shows the same convergence for one of the rat scans with a VOI enclosing the heart of the rat. Relative to the phantom reconstruction, the activity in the heart of the rat reconstruction 
converges slower, requiring over 20 sub-iterations before the total activity in the VIO stabilizes, compared to only 5 for the phantom reconstructions. The change in total counts in the VOI between subsequent subsets is comparable to that seen in the phantom reconstruction; the total counts within the VOI increases by $2 \%$ when all the projections are included in each iteration.

\section{I.3 Absolute activity calibration}

Based on the activity measurements of the $150 \mu \mathrm{L}$ used for calibration, with no corrections an absolute activity calibration coefficient of $0.841 \pm 0.003 \mathrm{kBq} /($ count $/ \mathrm{s})$ was calculated. With correction for attenuation only the calibration coefficient was $0.788 \pm 0.003 \mathrm{kBq} /($ count $/ \mathrm{s})$, and with broad-beam scatter correction $0.797 \pm 0.003 \mathrm{kBq} /($ count $/ \mathrm{s})$. The last of these was used for the analysis which follows.

\section{I.4 Amount of scatter and choice of DEW scatter coefficient, $k$}

For the cylindrical phantom experiment, the relative number of total counts observed in the downscatter window (97-125 keV) as a percentage of the counts in the photopeak window (126$154 \mathrm{keV}$ ), is shown in Figure 5-5, and follows the trend predicted for scatter to primary ratio modeled by (Hwang, Franc, Gullberg, \& Hasegawa, 2008). The downscatter to photopeak ratio increases with the size of the phantom from $12 \%$ ( $9 \mathrm{~mm}$ diameter) to $22 \%$ ( $52 \mathrm{~mm}$ diameter). The ratio levels off, approaching a value of approximately $25 \%$ for a volume of activity at the center of a $50 \mathrm{~mm}$ diameter cylinder of water (the largest that will fit on the scanner bed). For a point source, the number of counts observed in the downscatter window is still $9 \%$ of the number in the photopeak window, suggesting that camera scatter also contributes to the total scatter. In the in vivo scans, the ratio of counts in the downscatter window to counts in the photopeak window was $32 \%$; considerably higher than observed in phantom experiments. 


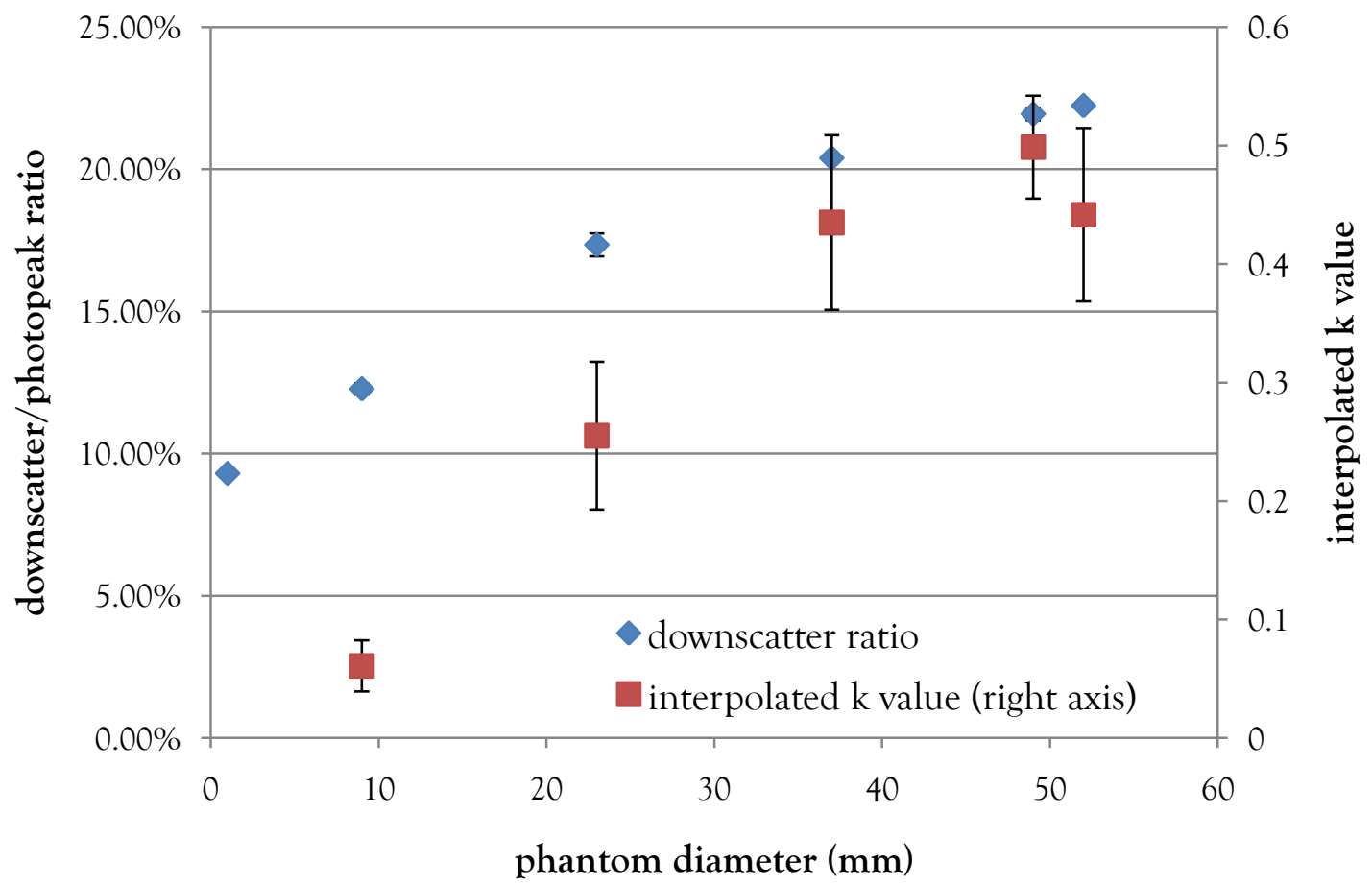

Figure 5-5. Left axis: ratio of total number of photons detected in the downscatter window (97-125 keV) to the total photons detected in the photopeak window (126-154 keV). Right axis: scatter fraction required for accurate dual-energy-window scatter correction, based on interpolation between attenuation correction alone $(k=0)$ and attenuation correction + DEW scatter correction with $\mathrm{k}=0.5$.

To estimate the optimal scatter multiplier $(\mathrm{k})$ for quantification with DEW scatter correction, phantoms were reconstructed with $\mathrm{k}=0.50$ and the estimated $\mathrm{k}$ value for correct quantification was determined by interpolation between the $\mathrm{k}=0.5$ reconstruction and the attenuation-correctiononly reconstruction. Figure 5-5 shows the k value calculated for each size of phantom. The value of $\mathrm{k}$ required for accurate quantification of a hot source in a cold background increases with the size of the phantom, but for phantoms larger than $35 \mathrm{~mm}$, the $\mathrm{k}$ value is constant within the uncertainty of the measurement. Based on these results, we chose $\mathrm{k}=0.45$ for the in vivo quantification of myocardial activity in rats, which measure about $35 \mathrm{~mm}$ from spine to sternum, and 45-50 mm left to right. 


\section{I.5 Phantom measurements}

The normalized absolute activity concentration for each of the phantoms is shown in Figure 5-6. With no correction for either scatter or attenuation, the measured activity concentration in the $9 \mathrm{~mm}$ phantom is $8 \%$ below the true value and decreases as the size of the phantom increases to $30 \%$ below the true value for the $52 \mathrm{~mm}$ phantom. With attenuation correction, the measured activity is overestimated by $<1 \%$ for the smallest phantom and by $5 \%$ for the three largest phantoms. The trend toward greater overestimation of the activity with increasing phantom size is consistent with increasing scatter contribution in larger phantoms. With attenuation and DEW scatter correction $(\mathrm{k}=0.45)$ the measured activity is slightly underestimated for the smaller phantoms $(95.5 \pm 0.4 \%$ and $97.2 \pm 0.6 \%)$, but accurate for the $37-52 \mathrm{~mm}$ phantoms: $99.5 \pm 0.7 \%$ of the true activity concentration. Broad-beam scatter correction results in a measured activity $99 \pm 1 \%$ of the true activity across all phantom sizes.

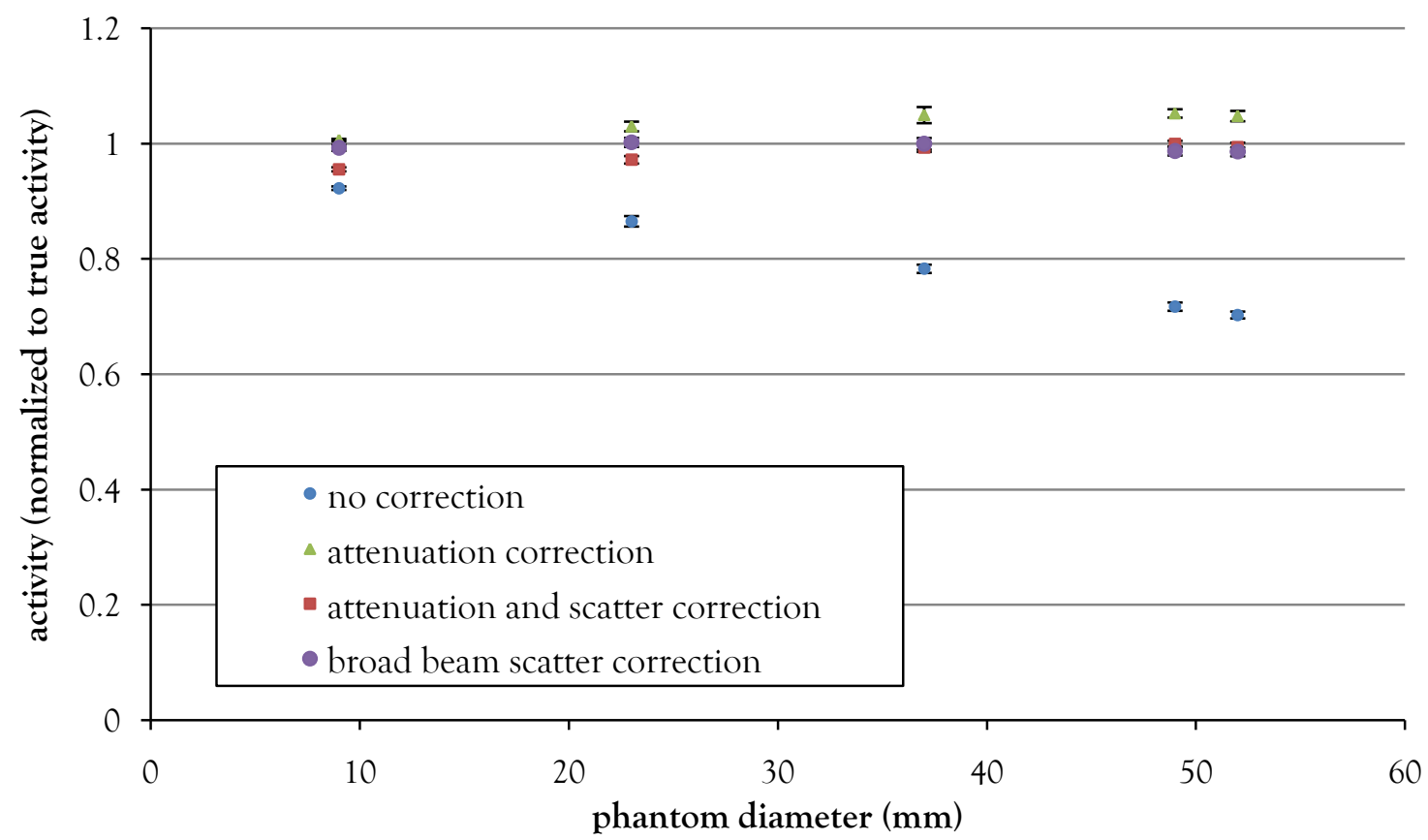

Figure 5-6. Activity concentrations normalized to true value, with no correction for attenuation, attenuation correction only, both attenuation and dual energy window scatter correction, and attenuation correction with reduced attenuation coefficient. 


\section{I.6 Heart-liver and heart-lung phantoms}

Although the phantom experiments showed that the increased ratio of scatter to primary counts was directly correlated with an increased need for scatter correction, the relationship between the total amount of scatter in the projection data and the influence of scatter on activity measured in a particular ROI was not direct. With cold water used to moisten a sponge (simulating the lungs) around the activity, we scanned the phantom with and without a large bottle of activity placed adjacent to the heart to simulate the liver. With this configuration, the total amount of activity in the downscatter window increased dramatically with the presence of activity in the 'liver'. The amount depended on how much of the liver was in the field of view, but using a phantom placement, liver activity concentration, and field of view that approximated the rat imaging setup, we observed a down-scatter fraction of $31 \%$, similar to that observed for the in vivo imaging. Despite the much higher scatter fraction, the total activity measured in a ROI enclosing the vial changed by less than $1 \%$ when the 'liver' activity was present. This was true for scans reconstructed with no correction as well as for those done with attenuation or scatter correction.

With activity in the 'lungs', the downscatter-to-photopeak count ratio decreased by $1 \%$ compared to 'lungs' that were moistened but contained no activity - from $17 \%$ to $16 \%$. With cold water in the lungs, DEW scatter corrected activity was $0.5 \%$ above the broad beam scatter corrected image. With activity in the lungs, the DEW corrected activity was $1 \%$ below the broad-beam scatter corrected reconstruction. Compared to the attenuation-corrected activity, the measured activity with broad-beam scatter correction was unaffected by the presence of background, the DEW corrected activity decreased by $1.5 \%$ with background activity. This suggests some difference in sensitivity to background activity between the two approaches to scatter correction, but the changes are not significant relative to the experimental uncertainty. 


\section{I.7 In vivo experiment}

The results of the in vivo measurements of activity uptake in the myocardium of five rats are shown in Table 5-1. Uncorrected, the activity measured using SPECT is $24 \pm 2 \%$ below the true value, by interpolation of the data observed in the water phantoms, this is equivalent to the amount of attenuation expected for a $35 \mathrm{~mm}$ diameter water phantom. Attenuation correction alone tends to overestimate activity by $5 \pm 3 \%$, consistent with the phantom experiments. Both approaches to scatter correction give results consistent with the activity measured by the well counter, $-3 \pm 3 \%$ and $-1 \pm 3 \%$ for DEW and broad-beam scatter correction respectively. The difference between the DEW and broad-beam correction was consistent with that seen in the phantom experiment with background activity, though both measurements agreed with the true value within the statistical uncertainty. (For the in vivo quantification, the uncertainties stated are the standard deviation across all five rats.)

Table 5-1. Tracer activity measured in rat myocardia, directly in a well counter and with SPECT.

\begin{tabular}{|c|c|c|c|c|c|}
\hline \multirow[t]{2}{*}{ Rat } & \multirow{2}{*}{$\begin{array}{c}\text { Ex vivo } \\
\text { measurement } \\
(\mathrm{kBq})\end{array}$} & \multicolumn{4}{|c|}{ "SPECT measurement $(\mathrm{kBq})$} \\
\hline & & $\begin{array}{l}\text { No } \\
\text { correction }\end{array}$ & AC only & $\begin{array}{c}\mathrm{AC}+\mathrm{SC} \\
(\mathrm{DEW} \mathrm{k}=0.45)\end{array}$ & $\begin{array}{c}\mathrm{AC}+\mathrm{SC} \\
\text { (broad beam) }\end{array}$ \\
\hline 1 & 1570 & $1192(-24 \%)$ & $1645(+5 \%)$ & $1518(-3 \%)$ & $1548(-1 \%)$ \\
\hline 2 & 1725 & $1274(-26 \%)$ & $1776(+3 \%)$ & $1643(-5 \%)$ & $1667(-3 \%)$ \\
\hline 3 & 1653 & $1270(-23 \%)$ & $1766(+7 \%)$ & $1625(-2 \%)$ & $1659(+0.3 \%)$ \\
\hline 4 & 1671 & $1242(-26 \%)$ & $1694(+1 \%)$ & $1572(-6 \%)$ & $1597(-4 \%)$ \\
\hline 5 & 1708 & $1347(-20 \%)$ & $1861(+9 \%)$ & $1720(+1 \%)$ & $1750(+2 \%)$ \\
\hline Aver & & $-24 \pm 2 \%$ & $+5 \pm 3 \%$ & $-3 \pm 3 \%$ & $-1 \pm 3 \%$ \\
\hline
\end{tabular}

As a fraction of the counts in the photopeak window, the activity in the downscatter window was much higher in the in vivo scans than in the phantom scans. For the $37 \mathrm{~mm}$ phantom, the ratio of downscatter counts to photopeak counts was just about 20\%; for the rats, it was more than $30 \%$. As with the phantom experiment with a hot liver, the increase in the apparent amount of scatter did not noticeably affect quantification. 


\subsection{Discussion}

The calibration factor was calculated with and without attenuation correction. Although the scatter is minimal $(<1 \%)$, attenuation, from the animal bed and from the source itself combine to a difference of about $5 \%$ and, therefore, must be included in the calibration for accurate quantification. The impact of attenuation would have been reduced if the bed was not in the field of view during the calibration scan. This was not done because the nanoSPECT scanner does not readily accommodate scanning without the bed and this would have required creation of a custom support for the calibration source. In addition, the self-attenuation of the source could be reduced by using a smaller source volume but, in practice, the calibration results for a more point-like source proved to be unrepeatable. The absolute activity calibration is specific to our reconstruction code. Our system matrix is scaled differently than the manufacturer supplied reconstruction software, and the value we measured cannot be directly applied to data reconstructed with other software.

With no correction for either attenuation or scatter, the activity was significantly underestimated by an amount that increased with the diameter of the phantom; for the largest phantom that would fit on the animal bed, the total underestimate of activity due to attenuation was $30 \%$. The total amount of attenuation observed when quantifying the activity in rat myocardia, was about $23 \%$, similar to that seen in a $35 \mathrm{~mm}$ (diameter) water phantom. Although the thorax of the rat is close to $50 \mathrm{~mm}$ across, the lungs which make up much of the tissue surrounding the heart and are less attenuating than water. The observed magnitudes of the scatter and attenuation were consistent with the Monte Carle calculations of (Hwang, Franc, Gullberg, \& Hasegawa, 2008) for single pinhole small animal SPECT. 
Attenuation correction alone was successful in restoring the lost activity for small phantoms, but also resulted in an overestimation of the activity of approximately 5\% in the larger phantoms and in in vivo experiments. Since attenuation correction alone resulted in consistent quantification across the three largest phantoms, attenuation correction alone is probably sufficient for most semiquantitative work where the subject are all $35 \mathrm{~mm}$ in diameter (water equivalent) or larger. The overestimate of the activity concentration in the larger phantoms is a result of the larger scatter contribution, which increases with the size of the phantom. To remove the residual phantom size dependence or for the most accurate recovery of absolute tracer concentration, both DEW and broad-beam scatter correction were successful, reducing the error to less than $3 \%$.

The scatter coefficient, $k$, required for DEW scatter correction is size dependent up to phantom diameters of $35 \mathrm{~mm}$, so for small phantoms, it is necessary to adjust the k-value for accurate quantification. Reconstruction using a fixed k-value of 0.45 accurately recovered the activity concentration in the larger phantoms, but underestimated activity concentrations by up to $5 \%$ in the smallest phantom $(10 \mathrm{~mm})$.

Broad-beam scatter correction using a scaling factor of 0.83 relative to narrow beam attenuation was the most successful method for accurately recovering tracer concentration across the full size range of phantoms we tested and in the in vivo measurements. The optimal scaling factor may have some material dependence (suggested by the outlying point for adipose tissue in our attenuation measurements), and may be slightly different in vivo than in uniform cylinders of water. However, since the effect of scatter is already small, the effect on quantification would likely be minimal.

The differences between DEW and broad-beam scatter correction we observed, were statistically insignificant for $37-52 \mathrm{~mm}$ phantoms ( $\mathrm{p}>0.05$, paired t-test), but for the smaller phantoms the DEW scatter correction underestimates the activity concentration by $4.5 \%$ ( $9 \mathrm{~mm}$ phantom) and 
$2.8 \%$ (23 $\mathrm{mm}$ phantom). The presence of background had a small but demonstrable effect on the DEW scatter correction. For quantification of hot regions against a cold background, this would lead us to prefer broad-beam scatter correction, being simpler to implement - not requiring an additional energy window to be acquired - and unaffected by background activity. In practice, for in vivo measurements, other experimental uncertainties, such as definition of volumes of interest or physiological variations or uncertainties due to tracer kinetics are likely to be much larger than any uncertainties introduced by scatter and, for ${ }^{99 \mathrm{~m}} \mathrm{Tc}$, a scatter correction method requiring an additional energy window is probably not justified. DEW scatter correction may still be useful for a non-quantitative study where the goal is to observe a cold lesion against a hot background, although we did not investigate this application.

The total amount of scatter observed in the projection data depends on the source activity distribution. In particular, hot regions at or just beyond the edge of the (axial) field for view may contribute substantially to the amount of scatter in the projection data. However, this scatter does not appear to significantly influence quantification with any of the corrections applied to the reconstruction.

In the only published nanoSPECT study of which we are aware where an attempt has been to perform absolute activity quantification in vivo, the researchers used a phantom of similar size to the subject to calibrate the absolute quantification of the scanner (Forrer, et al., 2006). While this method was successful in recovering an accurate measurement of the activity from the SPECT scans, we hope to demonstrate that with attenuation and scatter correction, quantitative activity can be recovered with a single sensitivity calibration, that is, without a calibration factor that depends on subject size and geometry. For example, when using rats in longitudinal studies lasting several weeks or more, the animals will grow significantly with their bodyweight potentially 
doubling over the duration of a given study, and it would be advantageous to be able to acquire quantitative measurements of tracer concentration without a size dependant calibration. As the animal grows the amount of attenuation and scatter increase proportionally, introducing further impetus for attenuation compensation even for semi-quantitative analysis of measurements made in the same animal over an extended period of time.

\subsection{Uncertainties and limitations}

The uncertainties in the in vivo measurements are greater than those observed in the phantom experiments. A number of factors contribute to this, including some uncertainty in the exact measurement of activity ex vivo and from the SPECT images. The VOI used for analysis was a cylindrical volume placed over the heart region. However it is possible that this region contains a small amount of activity external to the myocardium, either from the lungs, spill-over from the liver, or from the blood pool within the heart. Moreover, although the well counter used to measure the myocardium activity ex vivo was calibrated, it may exhibit some sensitivity to the distribution of the activity and the self-attenuation of the myocardium when counting activity.

The presence of bone in the rats may slightly increase the scatter contribution beyond that observed in the phantoms, though we did not evaluate this contribution and cannot say whether it is likely to be significant.

In this work we've looked only at hot spot quantification; quantification of cold regions within a hot background would require further investigation as to the success of the various scatter correction algorithms.

\subsection{Conclusions}

With full CT-based attenuation correction and dual-energy-window scatter or broad-beam scatter compensation, multiplexed multi-pinhole microSPECT can be used to obtain in-vivo measurement 
of absolute activity concentration in small animals with less than $5 \%$ error over a full range of sizes from a point source up to the capacity of the nanoSPECT rodent bed. Scatter correction provides some additional improvement in quantitative accuracy. Dual-energy-window and broad-beam attenuation scatter correction both give similar results, though the latter is slightly more straightforward, since it does not require an additional energy window, and does not depend on a heuristically determined and subject-size-dependent scatter fraction. 


\section{Reproducibility of cardiac rat SPECT data with attenuation correction}

\section{I Introduction}

In Chapter 4 we evaluated the reproducibility of NanoSPECT measurements of left ventricle blood perfusion, volumes, and ejection fractions in rats. This was done using the vendor reconstruction software without any attempt to correct for attenuation or scatter.

In clinical imaging, attenuation can introduce significant changes in this relative distribution causing attenuation artefacts, but the influence of attenuation on relative perfusion in rat imaging is unclear. In cardiac imaging we are often interested in the relative perfusion in the heart wherein uptake is evaluated as a percentage of the hottest portion of the heart. In addition, one of the strengths of small-animal imaging is the ability to serially image an animal. Over the course of several weeks, rats can increase substantially in weight; the rats used in this study grew from approximately $250 \mathrm{~g}$ to over $400 \mathrm{~g}$ in a period of less than five weeks. This growth increases the magnitude of attenuation and may influence the repeatability of cardiac measurements. 
Attenuation correction might offset these effects, but also has the potential to increase the noise in the reconstructed image and, therefore, potentially change the uncertainty and reproducibility of measurements.

The objective of this chapter, therefore, was to assess the impact of attenuation correction on the measured normal perfusion in the rat myocardia and on the intra- and inter-rat reproducibility of the perfusion measurements. In addition, we also consider the influence of applying a broad-beam attenuation coefficient for scatter compensation and hence more accurate quantification. In this chapter we reconstruct part of the same data sets with our own reconstruction software, both with and without attenuation correction.

\subsection{Methods}

For the ${ }^{99 \mathrm{~m}} \mathrm{Tc}$ scans, eight male Sprague-Dawley rats (age: $12 \mathrm{wks}$, initial weight: $255 \pm 22 \mathrm{~g}$ ), were injected with $2.0 \pm 0.3 \mathrm{mCi}^{99 \mathrm{~m}}$ Tc-tetrofosmin and anaesthetized with 2-3\% isoflorane. A circular cone-beam $45 \mathrm{kVp}$ CT scan was acquired for each rat, followed by SPECT acquisition with the following parameters: 48 projections, (2.5 min per projection), $92.5 \mathrm{~mm}$ multiplexed pinholes per head (APT2 apertures), energy window $140 \mathrm{keV} \pm 10 \%$, helical scan trajectory. The SPECT data used in this study was acquired beginning 50 min post injection, for a total scan duration of 30 min, or $150 \mathrm{sec} /$ projection. Each rat was scanned weekly over a period of five weeks, for a total of 40 scans included in this study. At the time of the final scan, the rats weighed on average $387 \pm 59 \mathrm{~g}$.

To compare the effects of attenuation correction in ${ }^{99 \mathrm{~m}} \mathrm{Tc}$ scans with that in scans where attenuation is likely to be more significant, we also retrospectively reprocessed a set of scans done using ${ }^{201} \mathrm{Tl}$. This study also contained eight male Sprague-Dawley rats with average initial weights of $283 \pm 30 \mathrm{~g}$, and average final weights of $444 \pm 34 \mathrm{~g}$. The rats were scanned over a period of five 
weeks. (Due to problems with some of the scan data, a full set of five scans was only available for three of the eight rats. Only three scans were available for the remaining five rats.) The rats were injected with $0.50 \pm 0.05 \mathrm{mCi}$ of ${ }^{201} \mathrm{Tl}$ and the scans began immediately following injection. The apertures, scan trajectory, and projection number and duration were the same as for the ${ }^{99 \mathrm{~m}} \mathrm{Tc}$ injected rats. Two energy windows of $64.8-79.2 \mathrm{keV}$ and $154.5-179.5 \mathrm{keV}$ were used with the counts from both windows combined in a single set of projections. (The decay of ${ }^{201} \mathrm{Tl}$ to ${ }^{201} \mathrm{Hg}$ by electron capture is followed by the emission of $K_{\alpha}$ or $K_{\beta}$ X-rays in the $69-83 \mathrm{keV}$ range. $10 \%$ of ${ }^{201} \mathrm{Tl}$ decays are followed by the emission of a $167.4 \mathrm{keV}$ gamma.)

In Chapter 4 the vendor software was used to reconstruct the scan data. In this chapter we use the software we developed that is capable of attenuation and scatter correction.

Attenuation correction was implemented as described in $\$ 3.3$. Scatter correction for the ${ }^{99 \mathrm{~m}} \mathrm{Tc}$ scans was also applied using the broad-beam attenuation approach discussed in the previous chapter. Since we have not evaluated the broad-beam attenuation coefficient for ${ }^{201} \mathrm{Tl}$, we did not apply scatter correction in the thallium reconstructions.

Reconstructed SPECT images were rotated in the transverse plane to align horizontal and vertical long axes (HLA and VLA, respectively) of the heart with the row and column vectors of the image and then imported into 4D-MSPECT (Invia, Ann Arbor, MI) for analysis. All images were analyzed using the default parameters supplied by Invia for analysis of rat cardiac scans. The hearts were manually aligned in the planes of the VLA and HLA and a 17 segment polar map of the myocardial perfusion was generated for each reconstruction. Relative perfusion for each segment is quoted as a percentage of that in the region of the myocardium where the measured perfusion is greatest. 
Because processing the scans requires a user to align the heart in the horizontal and vertical long axes and to set the valve plane, the intra- and inter-operator variability was assessed. Inter- and intrauser reproducibility of the 4D-MSPECT processing was assessed using three ${ }^{99 \mathrm{~m}} \mathrm{Tc}$ scans from different rats (with no attenuation or scatter correction). These scans were each processed 10 times by the same user who processed the data for the rest of the study. Each of the three scans was also processed by nine additional users to assess inter-user variability. These users were trained in medical physics and familiar with cardiac nuclear images but had no previous experience with the 4D-MSPECT software. Variability is quoted as the average standard deviation of relative segment perfusion.

Reproducibility was defined as described in \$4.2.2. To calculate inter-scan reproducibility, the scans were grouped by rat ( 5 scans per group), and the standard deviation of mean segment perfusion was calculated for each group and each segment, and then averaged over all eight rats. Inter-rat reproducibility was calculated by grouping the rats by scan number ( 8 rats per group), finding the standard deviations of mean segment perfusion, and then taking the average of the standard deviations. In the case of the ${ }^{201} \mathrm{Tl}$ studies where some scans were not available, the inter-rat variability was assessed over the 3 scans for which all 8 rats were available, and the inter-scan reproducibility was assessed with the 3 rats for which all 5 scans were available.

Finally, as part of this study we also evaluated the absolute tracer uptake as a percentage of the injected activity with no correction, attenuation alone, and attenuation and scatter correction. The total activity in the myocardium was determined using the total number of counts in a volume of interest enclosing the heart and converting to absolute activity using a pre-determined calibration constant as described in Chapter 5. 


\subsection{Results}

\subsection{Comparison to vendor software}

Perfusion maps for the reconstructions which used our reconstruction software were compared with perfusion maps for the reconstructions done using the vendor software (HiSPECT, Scivis, Germany). The relative perfusion for individual segments differed by between $-3.0 \%$ and $+2.7 \%$, and the average magnitude of the difference between the same segment for the vendor reconstruction and our reconstruction was $1.1 \pm 0.8 \%$.

\subsubsection{Comparison to normal database}

Comparison of perfusion data derived from our reconstruction (with no attenuation correction), shows agreement with the normal database calculated in Chapter 4. The average relative perfusion for fifteen of the segments was within $1 \mathrm{SD}$ of the normal database, and for the remaining 2 segments differed by $1.3 \mathrm{SD}$.

\subsubsection{Intra-and inter-operator variability}

Intra-user variability, assessed as the standard deviation of the reported relative perfusion for each segment over 10 repeated evaluations of the same scans, ranged from $0.5-2.1 \%$, with an average segment variability of $1.2 \%$. Inter-user variability was higher, from $1.0-5.3 \%$ with an average variability of $2.2 \%$.

\subsubsection{Effect of attenuation correction on relative perfusion}

The average perfusions of each segment and the standard deviations of the measured perfusions are shown with and without attenuation correction in Figure 6-1 for ${ }^{99 \mathrm{~m}} \mathrm{Tc}$ and Figure $6-2$ for ${ }^{201} \mathrm{Tl}$. As expected, regions of the myocardium on the septal and infero-septal wall tended to show increased perfusion following attenuation correction. Segments on the anterior wall tended to exhibit slightly decreased perfusion. The change in the measured relative perfusion of each segment is shown in Figure 6-3. The greatest increases in relative perfusion were observed in the basal segment of the 
infero-septal wall, where the relative perfusion increased by approximately $2 \%$ for the ${ }^{99 \mathrm{~m}} \mathrm{Tc}$ tetrofosmin scans and by $3 \%$ for the ${ }^{201} \mathrm{Tl}$ scans. The largest decrease in relative perfusion was observed in the middle of the anterior wall, where the relative perfusion decreased by $1.6-1.8 \%$ for the ${ }^{99 \mathrm{~m}} \mathrm{Tc}$ scans and $1.3-1.8 \%$ for the ${ }^{201} \mathrm{Tl}$ scans. Paired $\mathrm{t}$-tests on the tetrofosmin relative perfusion in each segment with and without attenuation correction showed significant changes $(\mathrm{p}<0.05)$ in 8 of 17 segments ( $\mathrm{p}<0.01$ in 6 segments), as noted on Figure 6-3. For the ${ }^{201} \mathrm{Tl}$ scans, all but four of the segments exhibited statistically significant changes in relative perfusion $(p<0.01)$ as a result of attenuation correction.

The uniformity of the ${ }^{99 \mathrm{~m}} \mathrm{Tc}$ perfusion map, measured as the standard deviation of the relative segment perfusion over all 17 segments for each data set analyzed, did not change significantly with attenuation correction or with attenuation and scatter compensation. Without correction, the uniformity was $7.3 \%$, with attenuation correction $7.2 \%(\mathrm{p}=0.2$, pared $\mathrm{t}$-test), and with attenuation and scatter uniformity was $7.5 \%$. For the ${ }^{201} \mathrm{Tl}$ scans, the uniformity was $7.1 \%$ with no correction and $6.8 \%$ after attenuation correction, which was statistically significant ( $\mathrm{p}=0.003$, paired t-test). 

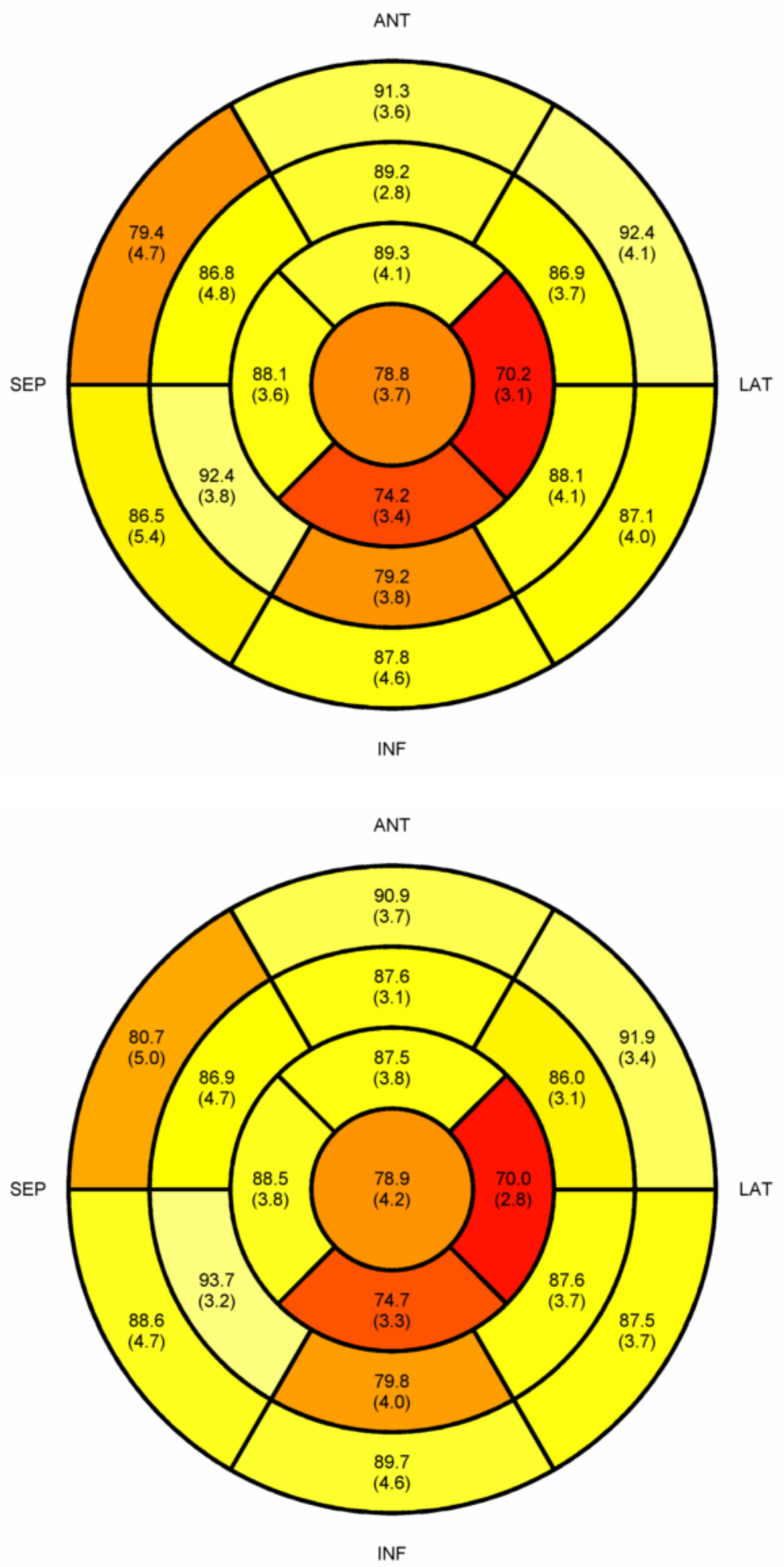

Figure 6-1. Average of normalized segment perfusion, without attenuation correction (top) and with attenuation correction (bottom) for ${ }^{99 \mathrm{~m}} \mathrm{Tc}$ scans. Values are given as percentages relative the region of maximum perfusion, with the standard deviation over all scans in brackets. 

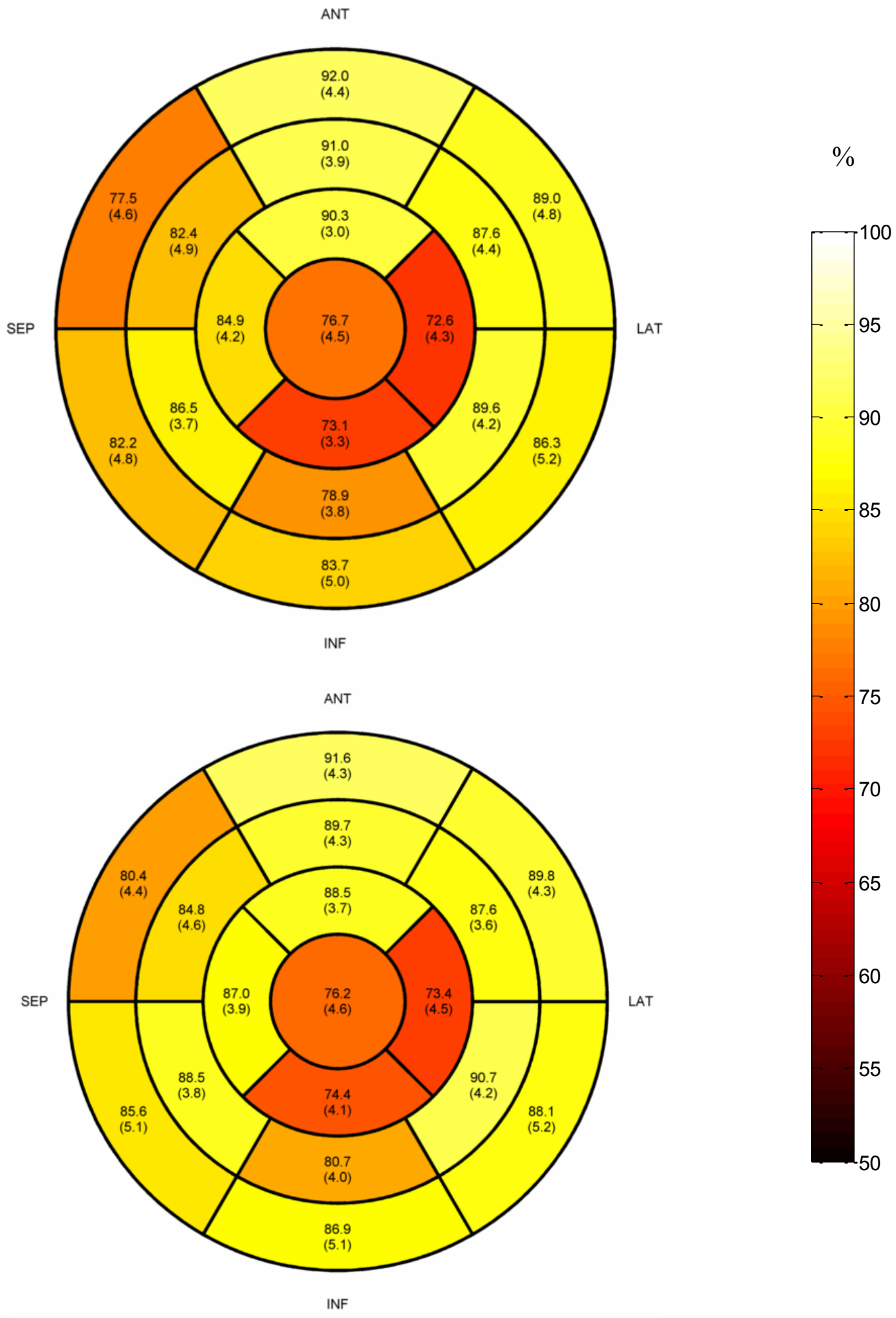

Figure 6-2. Average of normalized segment perfusion, without attenuation correction (top) and with attenuation correction (bottom) for ${ }^{201} \mathrm{Tl}$ scans. Values are given as percentages relative the region of maximum perfusion, with the standard deviation over all scans in brackets. 

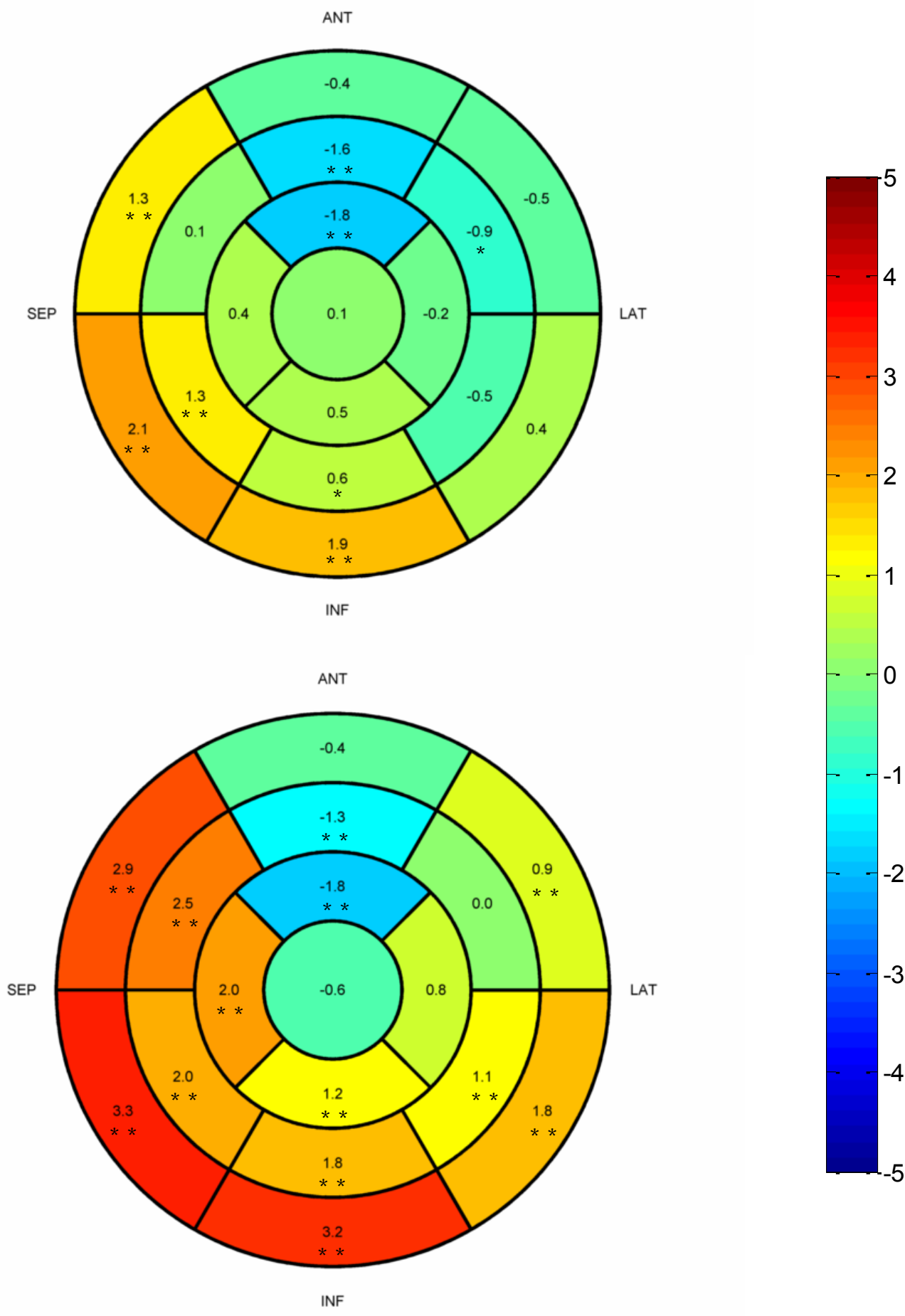

Figure 6-3. Change in relative perfusion of each segment as a result of attenuation correction for ${ }^{99 \mathrm{~m}} \mathrm{Tc}$ (top) and ${ }^{201}$ Tl (bottom). Segments where the change was significant $(\mathbf{p}<0.01$, paired $t$-test) are indicated $(* \mathbf{p}<0.05, * *$ $\mathbf{p}<\mathbf{0 . 0 1 )}$. 


\subsubsection{Effect of attenuation correction on reproducibility}

The segment perfusion reproducibility for the uncorrected, attenuation corrected, and attenuation and scatter corrected reconstructions of the ${ }^{99 \mathrm{~m}} \mathrm{Tc}$ scans are listed in Table 6-1. Evaluation of the inter-rat and inter-scan reproducibility, performed as per our previous work, shows that for both the inter-scan and inter-rat analysis, inclusion of attenuation correction results in no change to the reproducibility: $<0.05 \%$ change to average inter-scan reproducibility $(\mathrm{p}=0.49$, paired $\mathrm{t}$-test), $-0.2 \%$ change to average inter-rat reproducibility $(\mathrm{p}=0.06)$. Although CT-based attenuation correction does not improve reproducibility of cardiac perfusion measurements, neither does it degrade the reproducibility. Scatter correction likewise has only a small influence on reproducibility: $+0.2 \%$ change to average inter-scan reproducibility $(\mathrm{p}=0.02),+0.1 \%$ change to average inter-rat reproducibility $(\mathrm{p}=0.30)$.

The reproducibilities of the ${ }^{201} \mathrm{Tl}$ (Table 6-2) perfusion measurements exhibit similar behaviour. Neither the inter-scan nor inter-rat reproducibility is affected by attenuation correction: the change in reproducibility is $<0.05 \%$ for both ( $\mathrm{p}=0.41$ inter-scan; $\mathrm{p}=0.44$ inter-rat). The inter-scan reproducibility is about $0.9 \%$ poorer for ${ }^{201} \mathrm{Tl}$ than for ${ }^{99 \mathrm{~m}} \mathrm{Tc}$, but the inter-rat reproducibility is only about $0.1-0.3 \%$ worse.

Table 6-1. Range and average of segment perfusion reproducibility for ${ }^{99 \mathrm{~m}}$ Tc-tetrofosmin over 17 segments for uncorrected, attenuation corrected, and attenuation and scatter corrected reconstructions.

\begin{tabular}{lcl}
\hline \hline & Inter-scan reproducibility range (mean) & Inter-rat reproducibility range (mean) \\
\hline No correction & $2.1 \%-4.1 \%(2.7 \%)$ & $2.7 \%-5.4 \%(3.9 \%)$ \\
AC & $1.9 \%-3.8 \%(2.7 \%)$ & $2.8 \%-4.9 \%(3.7 \%)$ \\
AC+SC & $2.2 \%-3.6 \%(2.9 \%)$ & $2.8 \%-4.9 \%(3.8 \%)$ \\
\hline
\end{tabular}


Table 6-2. Reproducibilities for ${ }^{201} \mathrm{Tl}$ imaging.

\begin{tabular}{lcl}
\hline \hline & Inter-scan reproducibility range (mean) & Inter-rat reproducibility range (mean) \\
\hline No correction & $2.2 \%-5.9 \%(3.6 \%)$ & $2.6 \%-4.9 \%(4.0 \%)$ \\
AC & $2.4 \%-5.8 \%(3.6 \%)$ & $3.0 \%-4.8 \%(4.0 \%)$ \\
\hline
\end{tabular}

\subsubsection{Tracer uptake}

The average tetrofosmin uptake, calculated as a percentage of the activity measured in the heart divided by the total injected activity was $1.3 \pm 0.2 \%$ without attenuation correction, $1.8 \pm 0.3 \%$ after attenuation correction and $1.7 \pm 0.3 \%$ with both attenuation and scatter correction. The difference between the uncorrected and attenuation corrected average uptake was significant $(\mathrm{p}<0.01, \mathrm{t}$-test), but the difference between the attenuation corrected and the attenuation and scatter corrected uptake was not $(\mathrm{p}=0.12$, $\mathrm{t}$-test). Furthermore, the tracer uptake in the myocardium as a percentage of injected activity was correlated with the size of the rats (Pearson correlation coefficient: $\mathrm{r}=0.48$ for the uncorrected data, $\mathrm{r}=0.58$ for both the attenuation and attenuation and scatter corrected data), as shown in Figure 6-4. Other work using small animal PET has also demonstrated that myocardial blood flow in rats increases with age and weight (Lamoureux, et al., 2012). With no attenuation correction, the percentage uptake in the myocardium increased with the weight of the rats by $2.0 \pm 0.6 \% / \mathrm{kg}$ with no corrections, $3.4 \pm 0.8 \% / \mathrm{kg}$ with attenuation correction alone, and by $3.1 \pm 0.7 \% / \mathrm{kg}$ with attenuation and scatter correction. The slope of the difference between the attenuation corrected and uncorrected uptake versus weight was significantly different from zero $(\mathrm{p}<0.01)$, as was the slope of the difference between the $\mathrm{AC}+\mathrm{SC}$ and the uncorrected values $(\mathrm{p}<0.01)$. The slope of the difference between the attenuation and the $\mathrm{AC}+\mathrm{SC}$ values was also significant $(\mathrm{p}=0.02)$, but very small $(0.3 \% / \mathrm{kg})$. 


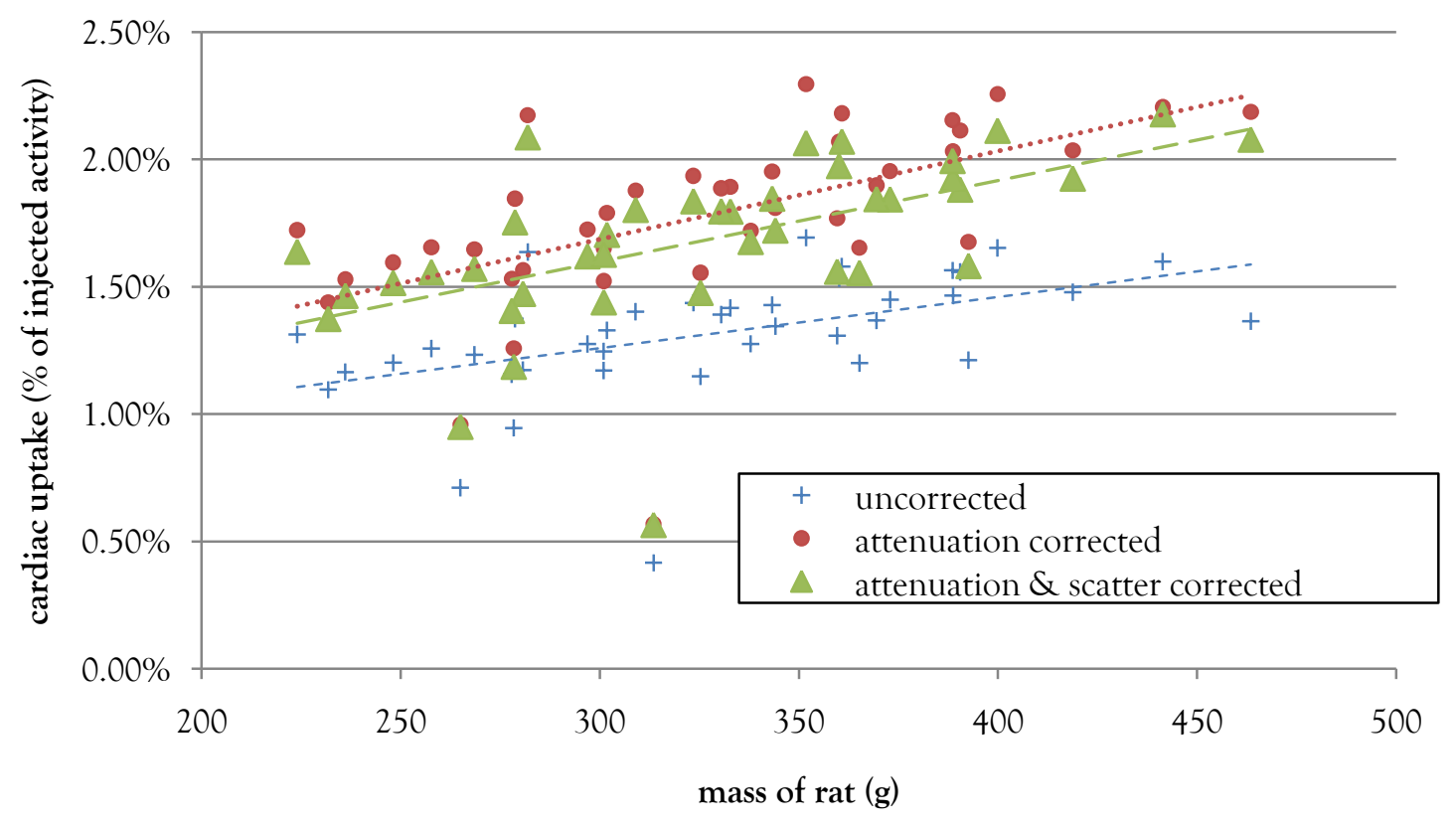

Figure 6-4. Total myocardial uptake as a percentage of injected dose for rats injected with ${ }^{99 m}$ Tc-tetrofosmin.

\subsection{Discussion}

The inter-scan and inter-rat reproducibilities measured with tetrofosmin in this study were within $0.1 \%$ of those observed in the previous reproducibility study, despite differences in the reconstruction and analysis software.

For the previous work we used the vendor software supplied with the nanoSPECT/CT system (HiSPECT, Mediso, Budapest, Hungary). For this study, all reconstruction, both with and without attenuation correction, was done using our own software. Our reconstruction includes an additional set of iterations of the reconstruction algorithm with all projections, so the OSEM subset scheme is not exactly identical.

The analysis software has also changed since the previous work. Previously, since only human cardiac dimensions were supported, we scaled the apparent size of the voxels of the rat images by a factor of 10 in all three dimensions. Because the 4D-MSPECT defaults for human hearts expects a wall thickness of $10 \mathrm{~mm}$, this is equivalent to assuming a $1 \mathrm{~mm}$ wall thickness for the rat hearts 
being analyzed. However, the default parameters for rat heart analysis supported by the current version of 4D-MSPECT assume an average wall thickness of $2 \mathrm{~mm}$. Additional changes to the 4DMSPECT software also required some rotation (in the axial plane) to be pre-applied before importing the images, since 4D-MSPECT does not permit rotation in the plane of the short axis.

The inter-operator reproducibility was assessed with a small number of scans and with operators who had not previously worked with the 4D-MSPECT analysis software. Therefore, the observed inter-user reproducibility - as high as $5.3 \%$ for one segment - was likely an upper bound that we would expect to be lower if measured with experienced users. The inter-rat and inter-scan reproducibilities should be interpreted in light of the average intra-user segment reproducibility of $1.2 \%$. Since the measurement of intra-user repeatability was done by repeatedly processing the same scan, memory may play a role and result in a lower variability than if scans were evaluated on different days.

Generally, the changes introduced to the perfusion map by attenuation correction are, qualitatively, exactly those which would be expected: the relative perfusion of the segments deeper in the rat the inferior and septal wall of the heart - increased slightly, while that of the more superficial anterior wall slightly decreased. Quantitatively, while the changes were statistically significant for a few segments of the ${ }^{99 m} \mathrm{Tc}$ studies (based on paired t-tests) and for most segments of the ${ }^{201} \mathrm{Tl}$ studies, they were small enough that we would not expect attenuation correction to make a meaningful difference to the evaluation of relative myocardial perfusion in rats. At the very least, the additional work and processing time are not justified by any improvement to the measurement of myocardial perfusion. However, neither does attenuation correction appear to degrade perfusion measurements by introducing additional noise. 
Attenuation correction makes a significant difference compared to no correction when quantitative tracer uptake is being measured. Particularly when quantifying relative changes over a period of time during which the animals grow significantly, attenuation correction is needed to prevent the increase in attenuation from reducing the apparent uptake. The difference between attenuation alone and attenuation plus scatter correction was measured to be statistically significant, although the absolute magnitude of the difference is unlikely to significantly influence outcomes in practice. Nevertheless, as we have shown in the previous chapter, using a reduced attenuation coefficient (i.e. for ${ }^{99 \mathrm{~m}} \mathrm{Tc}$ and water, $0.12 \mathrm{~cm}^{-1}$, rather than $0.15 \mathrm{~cm}^{-1}$ ), slightly improves the absolute quantification.

Although data from ${ }^{201} \mathrm{Tl}$ studies was not available to evaluate the quantitative accuracy of tracer uptake measurements, attenuation correction was seen to make a larger difference in the relative perfusion scans compared to tetrofosmin and so also is expected to be more important for quantification of ${ }^{201} \mathrm{Tl}$ than it is for ${ }^{99 \mathrm{~m}} \mathrm{Tc}$.

\subsection{Conclusion}

We have measured the impact of implementing CT-based attenuation correction in the reconstruction of rat myocardial perfusion scans, and demonstrated that the changes due to attenuation correction, though observable, are comparatively small. For large sample sizes, even small differences will be statistically significant, and this may need to be considered if results are compared between reconstructions that have been corrected for attenuation and those that haven't. However, attenuation correction is necessary for quantitative comparison between different time points in a longitudinal study where the animal grows significantly over the course of the study. 


\section{Conclusions}

\section{I Reproducibility of rat cardiac measurements using microSPECT}

\section{I.I Functional cardiac measurements}

With multiplexed, multi-pinhole microSPECT functional cardiac measurements in rats are very repeatable. In normal rats, we have shown that measurements of ejection fraction are repeatable with a standard deviation of $3 \%$ over multiple scans of the same rat or over different rats. Reproducibility is slightly degraded by myocardial infarction. Repeated scans of the same rat are only slightly less repeatable than those on normal rats.

\section{I.2 Semi-quantitative cardiac perfusion measurements}

We have created a normal database of the mean perfusion by segment for ${ }^{99 \mathrm{~m}} \mathrm{Tc}$-tetrofosmin and ${ }^{201} \mathrm{Tl}$ scans of rat myocardia. The repeatability of relative perfusion measurements for a single segment, expressed as for the functional measurements was approximately 3\% (inter-scan) and approximately $4 \%$ (inter-rat) for both ${ }^{99 \mathrm{~m}} \mathrm{Tc}$-tetrofosmin and ${ }^{201} \mathrm{Tl}$.

The reproducibility of the relative perfusion measurements is unaffected by attenuation correction. Attenuation correction did lead to small changes to the relative perfusion measurements, but for

${ }^{99 \mathrm{~m}}$ Tc-tetrofosmin, the changes were smaller than the measured reproducibility of the segment 
perfusion. Nevertheless, for large enough sample sizes, the changes may be significant. With a ${ }^{201} \mathrm{Tl}$ tracer, the changes to relative perfusion were larger than for ${ }^{99 \mathrm{~m}} \mathrm{Tc}$, and similar in magnitude to the reproducibility of the segment perfusion, suggesting that $\mathrm{AC}$ is of increased importance for ${ }^{201} \mathrm{Tl}$ where the primary emission energies are lower.

\subsection{Quantitative small animal microSPECT}

With careful calibration, absolute tracer concentration can be recovered from microSPECT images of phantoms with an error of less than $2 \%$. With attenuation correction alone, there is a residual dependence of the accuracy of the activity recovery on the phantom size. Dual-energy-window scatter correction is able to compensate for the size dependence, but requires a scatter coefficient to be specified for reconstruction which also depends on the phantom size. Using broad-beam attenuation correction, we were able to recover absolute activity concentration without having to specify any parameters which depend on the size of the phantom.

The accuracy of the same measurements made in vivo will be reduced somewhat by the tracer kinetics, but accurate quantification should was still achievable in vivo. We were able to measure total cardiac tracer uptake in rats with an error of less than $3 \%$ relative to well counter measurements of the rat hearts.

\subsection{When is attenuation (and scatter) correction necessary?}

The need for attenuation correction depends on the information one hopes to acquire from the SPECT scan. For cardiac imaging, attenuation correction does make a difference. However, for relative perfusion measurements using a ${ }^{99 \mathrm{~m}} \mathrm{Tc}$ based tracer, the changes are detectable with sensitive statistical tests, but small relative to other uncertainties in the relative perfusion measurements. The same holds true for ${ }^{201} \mathrm{Tl}$ images, although in the latter case, the magnitude of the changes to relative perfusion introduced by attenuation correction is slightly larger. If imaging 
were done using a tracer such as ${ }^{125}$ I-iodorotenone (27-35 keV X-rays) (VanBrocklin, Hanrahan, Enas, Nandanan, \& O'Neil, 2007) we would expect attenuation correction to be essential even for relative perfusion imaging.

Attenuation correction is necessary when the goal is to measure absolute tracer uptake, or in longitudinal studies on subjects which grow substantially over the course of the study. Scatter correction can slightly improve the accuracy of phantom measurements, and our measurements support using a reduced attenuation coefficient for scatter compensation. The accuracy achieved with this simple approach reduces uncertainties below the reproducibility of the measurements, suggesting that more complex methods which attempt to actually estimate the scatter contribution such as Monte Carlo methods or even energy-based methods like DEW correction are not necessary.

\subsection{Possible areas of future work}

\subsection{Other isotopes}

Quantitative accuracy has only been validated using ${ }^{99 \mathrm{~m}} \mathrm{Tc}$. Differences in the photon energies and the changes to the energy windows used would require the calibration to be re-measured for

isotopes other than ${ }^{99 \mathrm{~m}} \mathrm{Tc}$. Attenuation coefficients and scatter estimation and compensation would also need to be reassessed. Moreover, isotopes which emit photons with different energies introduce the possibility of scattered high energy photons being detected in lower energy windows which would modify the scatter correction needed for quantitative accuracy. In these situations, it is possible that methods other than the broad-beam and DEW correction may be required for accurate quantification. Isotopes with higher energy photons may also require modelling of photon penetration at the edges of the aperture which would modify the system matrix. 


\subsubsection{Other small animals}

The work in this thesis has focussed entire on rats - scans were done using the rat apertures and approximately rat-sized phantoms and the in-vivo work was done using rats. Since mouse models are also commonly used for cardiac studies and other research, extension to mice would be of interest. Based on the phantom measurements done in this work, it is unlikely that scatter correction would yield any improvement in quantification in mice. Also, as the effects are smaller in mice, there would be no expected impact of attenuation or scatter on relative perfusion measurements. However, although small in magnitude, attenuation would still affect the quantitative accuracy of mouse scans.

\subsubsection{D SPECT reconstruction}

In this research we have attempted to improve the accuracy of microSPECT imaging by modeling, as accurately as possible, the processes which degrade image quality in SPECT. One thing we have not modeled, however, is motion of the source during acquisition. Here we mean the physiological motion - breathing and heart beat - and not some gross displacement of the animal during the scan. Gating is used in cardiac SPECT acquisitions to reduce image blurring and to obtain functional cardiac data such as the volume of the left ventricle over the entire cardiac cycle.

The downside of gated acquisition is the reduction in the number of counts used to reconstruct each image for a given acquisition time. Alternatively, the length of acquisition could be increased by a factor corresponding to the number of gates used, but this substantially reduces the throughput of the SPECT scanner. Without increasing the acquisition time, the reduced number of counts in each gate results in significantly more noise in the reconstructed image which degrades the accuracy of the functional data obtained. 
It may be possible to reconstruct a clearer image of the heart wall by modelling the heart motion as part of the reconstruction, using the gated projection data to reconstruct a 'motion-frozen' image of the myocardium (Le Meunier, et al., 2011).

\subsubsection{Use of CT priors in reconstruction}

It may also be possible to improve SPECT images by using CT images to guide reconstruction (Gindi, Lee, Rangarajan, \& Zubal, 1991). Boundaries between organs visible on a CT scan are also likely to correspond to discontinuities in activity concentration in a SPECT scan. This may make it possible to improve the resolution of the reconstruction by using the CT information as a guide. This may also help to compensate for the partial volume effect (see following section) when attempting to measure activity in very small structures.

\subsubsection{Partial volume correction}

The partial volume effect arises when the blurring of small structures by the limited camera resolution decreases the apparent tracer concentration because a localized region of activity is 'smeared out' over a larger area in the reconstructed image. In the work presented in this thesis, the issue has been avoided by doing all quantification with structures significantly larger than the resolution of the camera. Partial volume has been well studied by other groups and incorporation of some method for partial volume correction into the analysis of the reconstructed SPECT images could improve quantitative measurement of small activity distributions. It is likely that it would be of more importance in smaller animals, such as mice, where the thickness of the myocardial wall is similar to the optimal camera resolution.

\subsubsection{Analytical assessment of multiplexed multi-pinhole field of view and degree of multiplexing}

In $\$ 3.5$ we conducted some simple simulations intended to establish how large of an activity distribution it was possible to reconstruct with the nanoSPECT/CT scanner and the APT2 
apertures. It can clearly be shown that once the distribution to be reconstructed exceeds a certain size, or alternatively once the degree of multiplexing on the detectors becomes too great, it becomes impossible to reconstruct accurately the original source distribution. In terms of linear algebraic formalism, the multiplexing may introduce a null space into the system matrix. We are not aware of any analytic analysis of the reconstruction of multiplexed multi-pinhole SPECT. While we do not expect analytic reconstruction to outperform iterative methods in the quality of the reconstructed images, chiefly because iterative methods allow for the pinhole dimensions, attenuation, and scatter to be modelled and incorporated into the reconstruction, an analytic approach would permit a more rigorous analysis of the limits of multiplexing, and the potential pitfalls, especially when imaging spatially extended subjects.

\subsection{Final Thoughts}

As a tool for basic research, multiplexed multi-pinhole SPECT provides a very sensitive, and as we have shown, a very accurate means of measuring tracer concentration in vivo. For cardiac functional quantification and semi-quantitative perfusion measurements in rats, we have assessed the reproducibility of microSPECT. The uncertainties we have measured are small, on the order of $3 \%$, and likely dominated by physiological variances rather than uncertainties introduced by the scanning process. Technical refinement of microSPECT is unlikely to lower these uncertainties. Only with low energy isotopes, possibly ${ }^{201} \mathrm{Tl}$, and almost certainly ${ }^{125} \mathrm{I}$, will rat cardiac measurements benefit from attenuation modelling.

For quantitative measurement of bio-distribution, the accuracy and uncertainties reported, on the order of $1-2 \%$ are also probably at the limit of what is achievable, having been measured with static distributions in phantoms, or very stable distributions (tetrofosmin) in vivo. Once attenuation and scatter are corrected, the residual uncertainties in the modelling of these phenomena are likely to 
be insignificant next to the uncertainties of physiological variability and non-static tracer distributions during a scan. Therefore, any future improvements to the accuracy are likely to come from more accurately modelling these parameters in reconstruction, as suggested above, based on CT-priors and/or 4-D modelling.

Finally, our work also notes, but did not fully address, two potential issues which should be considered in evaluating data acquired with microSPECT. These are the uncertainties in quantifying very small sources - where the uncertainties were on the order of $5-10 \%$ - and the problems with reconstructing non-sparse tracer distributions which lead to a very high degree of multiplexing. In the latter case, the multiplexing scheme used for this work may be unusable. Both of these issues should be addressed before using microSPECT for quantification in situations where they might lead to errors. 


\section{References}

Acton, P. D., Choi, S.-R., Plössl, K., \& Kung, H. F. (2002). Quantification of dopamine transporters in the mouse brain using ultra-high resolution single-photon emmision tomography. Eur J Nucl Med , 5, 691-698.

Anger, H. O. (1958). Scintillation Camera. Rev Sci Instrum, 29, 27-33.

Bal, G., \& Acton, P. D. (2006). Analytical derivation of the point spread function for pinhole collimators. Phys Med Biol , 51, 4923-4950.

Barrett, H. H. (1972). Fresnel zone plate imaging in nuclear medicine. J Nucl Med, 13, 382-385.

Barrett, H. H., Wilson, D. W., \& Tsui, B. M. (1994). Noise properties of the EM algorithm: I. Theory. Phys Med Biol, 39, 833-846.

Beekman, F. J., de Jong, H. W., \& van Geloven, S. (2002). Efficient fully 3-D iterative SPECT reconstruction with Monte Carlo-based scatter compensation. IEEE Trans Med Imaging , 21, 867877.

Beekman, F. J., McElroy, D. P., Berger, F., Gambhir, S. S., Hoffman, E. J., \& Cherry, S. R. (2002). Towards in vivo nuclear microscopy: iodine-125 imaging in mice using micro-pinholes. Eur J Nucl Med , 29, 933-938.

Beekman, F. J., van der Have, F., Vastenhouw, B., van der Linden, A. J., van Rijk, P. P., Burbach, P. H., et al. (2006). U-SPECT-I: A novel system for submillimeter resolution tomography with radiolabled molecules in mice. J Nucl Med , 46, 1194-1200.

Bellini, S., Piacentini, M., Cafforio, C., \& Rocca, F. (1979). Compensation of tissue absorbtion in emission tomography. IEEE Trans Acoust Speech Signal Process , 27, 213-218.

Bioscan. (2007). NanoSPECT/CT Preclinical Imager: Technical Brochure EF Specifications Guide. Washington, DC: Bioscan, Inc.

Blankespoor, S. C., Wu, X., Kalki, K., Brown, J. K., Tang, H. R., Cann, C. E., et al. (1996). Attenuation correction of SPECT using X-ray CT on an emission transmission system: Myocardial perfusion assesment. IEEE Trans Nucl Sci, 43 (4), 2263-2274.

Bloch, P., \& Sanders, T. (1973). Reduction of the effects of scattered raditaion on a sodium iodide imaging system. J Nucl Med , 14 (2), 67-72.

Bocher, M., Blevis, I. M., Tsukerman, L., Shrem, Y., Kovalski, G., \& Volokh, L. (2010). A fast cardiac gamma camera with dynamic SPECT capabilities: design, system validation and future potential. Eur J Nucl Med Mol Imaging, 37, 1887-1902.

Brooks, R. A., \& di Chiro, G. (1976). Beam hardening in x-ray reconstructive tomography. Phys Med Biol, 21, 390-398.

Budinger, T. F., \& Gullberg, G. T. (1974). Three-dimensional reconstruction in nuclear medicine emission imaging. IEEE Trans Nucl Sci , 21, 2-20. 
Byrne, C. L. (1996). Block-iterative methods for image reconstruction from projections. IEEE Trans Image Process , 5, 792-794.

Cao, L., \& Peter, J. (2012). Slit-slat collimator equipped gamma camera for whole-mouse SPECTCT imaging. IEEE Trans Nucl Sci, 59, 530-536.

Cao, Z., Bal, G., Accorsi, R., \& Acton, P. D. (2005). Optimal number of pinholes in multi-pinhole SPECT for mouse brain imaging - a simulation study. Phys Med Biol , 50, 4609-4624.

Chang, L.-T. (1978). A method for attenuation correction in radionuclide computed tomography. IEEE Trans Nucl Sci , 25, 638-643.

Chen, C.-L., Wang, Y., Lee, J. J., \& Tsui, B. M. (2009). Toward quantitative small animal pinhole SPECT: assessment of quantitation accuracy prior to image compensations. Mol Imaging Biol , 11, 195-203.

Constantinesco, A., Choquet, P., Monassier, L., Israel-Jost, V., \& Mertz, L. (2005). Assessment of left ventricular perfusion, volumes, and motion in mice using pinhole gated SPECT. J Nucl Med, 46, 1005-1011.

Croteau, E., Bénard, F., Cadorette, J., Gauthier, M.Ė., Aliaga, A., Bentourkia, M., et al. (2003). Quantitative gated PET for the assessment of left ventricular function in small animals. J Nucl Med, $44,1655-1661$.

Dawson, T. H. (2001). Similitude in the cardiovascular system of mammals. J Exp Biol , 204, 395 407.

de Jong, H. W., \& Beekman, F. J. (2001). Rapid SPECT simulation of downscatter in non-uniform media. Phys Med Biol, 46, 621-635.

Feldkamp, L. A., Davis, L. C., \& Kress, J. W. (1984). Practical cone-beam algorithm. J Opt Soc Am, $1,612-619$.

Feng, B., Chen, M., Bai, B., Smith, A. M., Austin, D. W., Mintzer, R. A., et al. (2010). Modeling of the point spread function by numerical calculations in single-pinhole and multipinhole SPECT reconstruction. IEEE Trans Nucl Sci, 57, 173-180.

Fessler, J. A. (1994). Penalized weighted least-squares image reconstruction for positron emission tomography. IEEE Trans Med Imaging , 13, 290-300.

Fishbein, M. C., Maclean, D., \& Maroko, P. R. (1978). Experimental myocardial infarction in the rat. Am J Pathol, 1978, 57-70.

Forrer, F., Valkema, R., Bernard, B., Schramm, N. U., Hoppin, J. W., Rolleman, E., et al. (2006). In vivo radionuclide uptake quantification using a multi-pinhole SPECT system to predict renal function in small animals. Eur J Nucl Med Mol Imaging , 33, 1214-1217.

Franc, B. L., Acton, P. D., Mari, C., \& Hasegawa, B. H. (2008). Small-animal SPECT and SPECT/CT: Important tools for preclinical investigation. J Nucl Med , 49, 1651-1663. 
Frey, E. C., \& Tsui, B. M. (1993). A practical method for incorporating scatter in a projectorbackprojector for accurate scatter compensation in SPECT. IEEE Trans Nucl Sci , 40, 1107-1116.

Funk, T., Després, P., Barber, W. C., Shah, K. S., \& Hasegawa, B. H. (2006). A multipinhole small animal SPECT system with submillimeter spatial resolution. Med Phys , 33, 1259-1268.

Furenlid, L. R., Wilson, D. W., Chen, Y.-C., Kim, H., Pietraski, P. J., Crawford, M. J., et al. (2004). FastSPECT II: A second-generation high-resolution dynamic SPECT imager. IEEE Trans Nucl Sci, 51, 631-635.

Gieles, M., de Jong, H. W., \& Beekman, F. J. (2002). Monte Carlo simulations of pinhole imaging accelerated by kernel-based forced detection. Phys Med Biol , 47, 1853-1867.

Gilbert, P. (1972). Iterative methods for the three-dimensional reconstruction of an object from projections. J Theor Biol , 36, 105-117.

Gindi, G. R., Arendt, J., Barrtt, H. H., Chiu, M. Y., Ervin, A., Giles, C. L., et al. (1982). Imaging with rotating slit collimators and rotating collimators. Med Phys , 9, 324-339.

Gindi, G., Lee, M., Rangarajan, A., \& Zubal, I. G. (1991). Bayesian reconstruction of functional images using registered anatomical images as priors. Information processing in medical imaging: 12th International Conference (pp. 121-131). Wye, UK: Springer-Verlag.

Gordon, R., Bender, R., \& Herman, G. T. (1970). Algebraic reconstruction techniques (ART) for three-dimensional electron microscopy and x-ray photography. J Theor Biol , 29, 471-480.

Gullberg, G. T., Heusman, R. H., Malko, J. A., Pelc, N. J., \& Budinger, T. F. (1986). An attenuated projector-backprojector for iterative SPECT reconstruction. Phys Med Biol , 30, 799-816.

Hasenfuss, G. (1998). Animal models of human cardiovascular disease, heart failure, and hypertrophy. Cardiovasc Res , 48, 60-76.

Holt, J. P., Rhode, E. A., \& Kines, H. (1968). Ventricular volumes and body weight in mammals. Am J Physiol , 215, 704-715.

Hsieh, J., Molthen, R. C., Dawson, C. A., \& Johnson, R. H. (2000). An iterative approach to the beam hardening correction in cone beam CT. Med Phys , 27, 23-29.

Hubbell, J. H., \& Seltzer, S. M. (2004). Tables of X-Ray Mass Attenuation Coefficients and Mass EnergyAbsorbtion Coefficients from $1 \mathrm{keV}$ to $20 \mathrm{MeV}$ for Elements $Z=1$ to 92 and 48 Additional Substances of Dosemetric Interest. (National Institute of Standards and Technology (NIST)) Retrieved 04 26, 2013, from http://www.nist.gov/pml/data/xraycoef/index.cfm

Hudson, H. M., \& Larkin, R. S. (1994). Accelerated image reconstruction using ordered subsets of projection data. IEEE Trans Med Imaging , 13, 601-609.

Hwang, A. B., \& Hasegawa, B. H. (2005). Attenuation correction for small animal SPECT imaging using x-ray CT data. Med Phys , 32, 2799-2005. 
Hwang, A. B., Franc, B. L., Gullberg, G. T., \& Hasegawa, B. H. (2008). Assessment of the sources of error affecting the quantitative accuracy of SPECT imaging in small animals. Phys Med Biol , 53, $2233-2252$.

Hwang, A. B., Taylor, C. C., VanBrocklin, H. F., Dae, M. W., \& Hasegawa, B. H. (2006). Attenuation correction of small animal SPECT images acquired with 125I-Iodorotenone. IEEE Trans Nucl Sci, 53, 1213-1220.

Ishizu, K., Mukai, T., Yonekura, Y., Pagani, M., Fujita, T., Magata, Y., et al. (1995). Ultra-high resolution SPECT system using four pinhole collimators for small animal studies. J Nucl Med , 36, 2282-2287.

Jaszczak, R. J., Coleman, R. E., \& Whitehead, F. R. (1981). Physical factors affecting quantitative measurements using camera-based single photon emission computed tomography (SPECT). IEEE Trans Nucl Sci , 28, 69-80.

Jaszczak, R. J., Greer, K. L., Floyd, C. E., Harris, C. C., \& Coleman, R. E. (1984). Improved SPECT quantification using compensation for scattered photons. J Nucl Med , 25 (8), 893-900.

Jaszczak, R. J., Li, J., Wang, H., Zalutsky, M. R., \& Coleman, R. E. (1994). Pinhole collimation for ultra-high-resolution, small-field-of-view SPECT. Phys Med Biol, 39, 425-437.

Kak, A., \& Slaney, M. (1988). Principles of Computerized Tomographic Imaging. New York: IEEE Press.

Koral, K. F., Swailem, F. M., Buchbinder, S., Clinthorne, N. H., Rogers, W. L., \& Tsui, B. M. (1990). SPECT Dual-Energy-Window Compton correction: scatter multiplier required for quantification. J Nucl Med , 31, 90-98.

Lamoureux, M., Thorn, S., Dumouchel, T., Renaud, J. M., Klein, R., Mason, S., et al. (2012). Uniformity and repeatability of normal resting myocardial blood flow in rats using [13N]-ammonia and small animal PET. Nucl Med Commun, 33, 917-925.

Larsson, S., \& Israelsson, A. (1982). Considerations on system design, implementation, and computer processing in SPECT. IEEE Trans Nucl Sci , 29 (4), 1331-1342.

Le Meunier, L., Slomka, P. J., Dey, D., Ramesh, A., Thomson, L. E., Hayes, S. W., et al. (2011). Motion frozen 18F-FDG cardiac PET. J Nucl Cardiol , 18, 259-299.

Li, J., Jaszczak, R. J., \& Coleman, R. E. (1995). Quantitative small field-of-view pinhole SPECT imaging: initial evaluation. IEEE Trans Nucl Sci , 42, 1109-1113.

Manglos, S. H., Jaszczak, R. J., \& Floyd, C. E. (1988). Weighted back-projection implemented with a non-uniform attenuation map for improved SPECT quantification. IEEE Trans Nucl Sci , 35, 625 628.

McElroy, D. P., MacDonald, L. R., Beekman, F. J., Wang, Y., Patt, B. E., Iwanczyk, J. S., et al. (2002). Performance evaluation of A-SPECT: A high resolution desktop pinhole SPECT system for imaging small animals. IEEE Trans Nucl Sci , 49, 2139-2147. 
Meikle, S. R., Fulton, R. R., Eberl, S., Dahlbom, M., Wong, K.-P., \& Fulham, M. J. (2001). An investigation of coded aperture imaging for small animal SPECT. IEEE Trans Nucl Sci , 48, 816-821.

Meikle, S. R., Hutton, B. F., \& Bailey, D. L. (1994). A transmission-dependent method for scatter correction in SPECT. J Nucl Med , 35, 360-367.

Meikle, S. R., Kench, P., Weisenberger, A. G., Wojcik, R., Smith, M. F., Majewski, S., et al. (2002). A prototype coded aperture detector for small animal SPECT. IEEE Trans Nucl Sci , 49, 2167-2171.

Miller, B. W., Furenlid, L. R., Moore, S. K., Barber, H. B., Nagarkar, V. V., \& Barrett, H. H. (2009). System integration of FastSPECT III, a dedicated SPECT rodent-brain imager based on BazookaSPECT detector technology. 2009 IEEE Nuclear Science Symposium Conference Record, (pp. 4004-4008).

Mok, G. S., Tsui, B. M., \& Beekman, F. J. (2011). The effects of object activity distribution on multiplexing multi-pinhole SPECT. Phys Med Biol , 56, 2635-2650.

Mok, G. S., Wang, Y., \& Tsui, B. M. (2009). Quantification of the multiplexing effects in multipinhole small animal SPECT - a simulation study. IEEE Trans Nucl Sci , 56, 2636-2643.

Nahrendorf, M., Wiesmann, F., Hiller, K.-H., Hu, K., Waller, C., Ruff, J., et al. (2001). Serial cinemagnetic resonance imaging of left ventricular remodeling after myocardial infarction in rats. $J$ Magn Reson Imaging , 14, 547-555.

Nakajima, K., Higuchi, T., Taki, J., Kawano, M., \& Tonami, N. (2001). Accuracy of ventricular volume and ejection fraction measured by gated myocardial SPECT: comparison of 4 software programs. J Nucl Med , 42, 1571-1578.

Natterer, F. (2001). Inversion of the attenuated Radon transform. Inverse Prob , 17, 113-119.

Nuyts, J., Vunckx, K., Defrise, M., \& Vanhove, C. (2009). Small animal imaging with multi-pinhole SPECT. Methods , 48, 83-91.

Ogawa, K., Harata, Y., Ichihara, T., Kubo, A., \& Hashimoto, S. (1991). A practical method for position-dependent Compton-scatter correction in single photon emission CT. IEEE Trans Med Imaging , 408-412.

Ogawa, K., Kawade, T., Nakamura, K., Kubo, A., \& Ichihara, T. (1998). Ultra high resolution pinhole SPECT for small animal study. IEEE Trans Nucl Sci , 45, 3122-3126.

Palmer, J., \& Wollmer, P. (1990). Pinhole emission computed tomography: method and experimental evaluation. Phys Med Biol, 35, 339-350.

Schramm, N. U., Ebel, G., Engeland, U., Schurrat, T., Béhé, M., \& Behr, T. M. (2003). Highresolution SPECT using multipinhole collimation. IEEE Trans Nuc Sci , 50 (3), 315-320.

Smith, M. F., \& Jaszczak, R. J. (1998). An analytic model of pinhole aperture penetration for 3D pinhole SPECT image reconstruction. Phys Med Biol, 43, 761-775. 
Strydhorst, J. H., Leenen, F. H., Ruddy, T. D., \& Wells, R. G. (2011). Reproducibility of serial left ventricle perfusion, volume, and ejection fraction measurements using multiplexed multipinhole SPECT in healthy rats and rats after myocardial infarction. J Nucl Med , 52, 1285-1292.

Tanaka, E., Toyama, H., \& Murayama, H. (1984). Convolutional image reconstruction for quantitative single photon emission computed tomography. Phys Med Biol , 29, 1489-1499.

Tretiak, O., \& Metz, C. (1980). The exponential Radon transform. SIAM J Appl Math , 39, 341 353.

Tsui, B. M., Gullberg, G. T., Edgerton, E. R., Ballard, J. G., Perry, J. R., McCartney, W. H., et al. (1989). Correction of nonuniform attenuation in cardiac SPECT imaging. J Nucl Med , 30, 497507.

Tuy, H. K. (1983). An inversion formula for cone-beam reconstruction. SIAM J Appl Math , 43, 546552.

van der Have, F., Vastenhouw, B., Ramakers, R. M., Branderhorst, W., Krah, J. O., Ji, C., et al. (2009). U-SPECT-II: An ultra-high-resolution device for molecular small-animal imaging. J Nucl Med , 50, 599-605.

VanBrocklin, H. F., Hanrahan, S. M., Enas, J. D., Nandanan, E., \& O'Neil, J. P. (2007). Mitochondrial avid radioprobes. Preparation and evaluation of $7^{\prime}(\mathrm{Z})-[125 \mathrm{I}]$ iodorotenone and $7^{\prime}(\mathrm{Z})$ [125I]iodorotenol. Nucl Med Biol , 34, 109-116.

Vanhove, C., Defrise, M., Bossuyt, A., \& Lahoutte, T. (2011). Improved quantification in multiplepinhole SPECT by anatomy-based reconstruction using microCT information. Eur J Nucl Med Mol Imaging , 38, 153-165.

Vanhove, C., Defrise, M., Bossuyt, A., \& Lahoutte, T. (2009). Improved quantification in singlepinhole and multiple-pinhole SPECT using micro-CT information. Eur J Nucl Med Mol Imaging, 36, 1049-1063.

Vanhove, C., Lahoutte, T., Defrise, M., Bossuyt, A., \& Franken, P. R. (2005). Reproducibility of left ventricular volume and ejection fraction measurements in rat using pinhole gated SPECT. Eur $J$ Nucl Med Mol Imaging , 32, 211-220.

Vogel, R. A., Krich, D., LeFree, M., \& Steele, P. (1978). A new method of multiplanar emission tomography using a seven pinhole collimator and an Anger scintillation camera. J Nucl Med , 19, 648-654.

Vunckx, K., Suetens, P., \& Nuyts, J. (2008). Effect of overlapping projections on reconstruction image quality in multipinhole SPECT. IEEE Trans Med Imaging , 27, 972-983.

Walrand, S., Jamar, F., de Jong, M., \& Pauwels, S. (2005). Evaluation of novel whole-body highresolution rodent SPECT (Linoview) based on direct acquisition of linogram projections. $J$ Nucl Med , 46, 1872-1880. 
Weber, D. A., Ivanovic, M., Franceschi, D., Strand, S.-E., Erlandsson, K., Franceschi, M., et al. (1994). Pinhole SPECT: an approach to in vivo high resolution SPECT imaging in small laboratory animals. J Nucl Med, 35, 342-348.

Wells, R. G., Celler, A., \& Harrop, R. (1998). Analytical calculation of photon distributions in SPECT projections. IEEE Trans Nucl Sci , 45, 3202-3214.

Wilson, D. W., Tsui, B. M., \& Barrett, H. H. (1994). Noise properties of the EM algorithm: II. Monte Carlo simulations. Phys Med Biol , 39, 847-871.

Wise, R. G., Huang, C. L.-H., Gresham, G. A., Al-Shafei, A. I., Carpenter, T. A., \& Hall, L. D. (1998). Magnetic resonance imaging analysis of left ventricular function in normal and spontaneously hypertensive rats. J Physiol , 513, 873-887.

Wu, C., van der Have, F., Vastenhouw, B., Dierckx, R. A., Paans, A. M., \& Beekman, F. J. (2010). Absolute quantitative total-body small-animal SPECT with focussing pinholes. Eur J Nucl Med Mol Imaging , 37, 2127-2135.

Wu, M. C., Tang, H. R., Gao, D. W., Ido, A., O'Connell, J. W., Hasegawa, B. H., et al. (2000). ECG-gated pinhole SPECT in mice with millimeter spatial resolution. IEEE Trans Nucl Sci , 47, 1218-1221.

Xu, Y., Hayes, S., Ali, I., Ruddy, T. D., Wells, R. G., Berman, D. S., et al. (2010). Automatic and visual reproducibility of perfusion and function measurements for myocardial perfusion SPECT. $J$ Nucl Cardiol, 17, 1050-1057.

Yanch, J. C., Flower, M. A., \& Webb, S. (1990). Improved quantification of radionuclide uptake using deconvolution and windowed subtraction techniques for scatter compensation in single photon emission computed tomography. Med Phys , 17, 1011-1022.

Yukihiro, M., Inoue, T., Iwasaki, T., Tomiyoshi, K., Erlandsson, K., \& Endo, K. (1996). Myocardial infarction in rats: high-resolution single-photon emission tomographic imaging with a pinhole collimator. Eur J Nucl Med , 23, 896-900.

Ziadi, H., \& Koral, K. F. (2004). Scatter modelling and compensation in emission tomography. Eur J Nucl Med Mol Imaging , 31, 761-782. 